FINAL REPORT

\title{
Measurement and Instrumentation Techniques for Monitoring Plutonium and Uranium Particulates Released from Nuclear Facilities
}

\author{
Anthony V. Nero, Jr. \\ Energy \& Environment Division \\ Lawrence Berkeley Laboratory \\ University of California \\ Berkeley, CA 94720
}

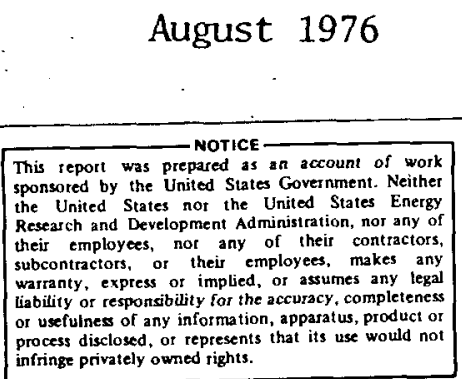

This document is

PUBLICLY RELEASADLR

Banin Stesle.

Authorizing Official

Date: $10 / 31 / 16$

Supported by the Environmental Protection Agency through Interagency Agreement D5-E681 with the Energy Research and Development Administration:

Subagreement 77 BCK, major task 2 


\section{DISCLAIMER}

This report was prepared as an account of work sponsored by an agency of the United States Government. Neither the United States Government nor any agency Thereof, nor any of their employees, makes any warranty, express or implied, or assumes any legal liability or responsibility for the accuracy, completeness, or usefulness of any information, apparatus, product, or process disclosed, or represents that its use would not infringe privately owned rights. Reference herein to any specific commercial product, process, or service by trade name, trademark, manufacturer, or otherwise does not necessarily constitute or imply its endorsement, recommendation, or favoring by the United States Government or any agency thereof. The views and opinions of authors expressed herein do not necessarily state or reflect those of the United States Government or any agency thereof. 


\section{DISCLAIMER}

Portions of this document may be illegible in electronic image products. Images are produced from the best available original document. 
The purpose of this work has been an analysis and evaluation of the state-ofthe-art of measurement and instrumentation techniques for monitoring plutoniun and uranium particulates released from nuclear facilities. The occurrence of plutonium and uranium in the nuclear fuel cycle, the corresponding potential for releases, associated radiological protection standards and monitoring objectives are discussed. Techniques for monitoring via decay radiation from plutonium and uranium isotopes are presented in detail, emphasizing air monitoring, but a1so including soil sampling and survey methods. Additionally, activation and mass measurement techniques are discussed. The availability and prevalence of these various techniques are summarized. Finally, possible improvements in monitoring capabilities due to alterations in instrumentation, data analysis, or programs are presented. 


\section{$-v-$ \\ Acknowledgments}

Participants in the Survey of Instrumentation for Environmental Monitoring, and other members of Lawrence Berkeley Laboratory's Energy and Environment Division, gave substantial support to this work. Dick A. Mack and Robert J. Budnitz gave major assistance, both in defining the coverage of the work and in reviewing this final report. In addition, Prem S. Bhardwaja, Frederick S. Goulding, Craig D. Hollowe11, Billy W. Loo, and George A. Morton made valuable conments on the manuscript.

Many persons outside the Laboratory were very helpful, both in discussing their own work and in providing information on other work and on monitoring methods utilized at their own institutions. We are grateful for their assistance and would like to acknowledge particularly the courtesy afforded us by the following individuals during visits to their laboratories and facilities:

Environmental Protection Agency, Las Vegas,

Environmental Monitoring and Support Laboratory:

E. W. Bretthauer

J. W. Mullins

D. L. Stevenson

Office of Radiation Programs:

R. L. Douglas

Battelle Pacific Northwest Laboratón:

N. E. Ballou

J. J. Stoffels

Idaho National Engineering Laboratory,

ERDA Health Services Laboratory:

C. W. Sil1

W. L. Polzer

Chemical Processing Plant (A11ied Chemical Corp.):

M. L. Arave

Los Alamos Scientific Laboratory (Univ. of Calif.):

J. C.. Elder

D. C. Gray

M. I. Tillery 
-vi-

Rocky Flats Plant (Rockwell International):

M. R. Boss

J. A. Hayden

C. G. Haynes

C. H. Johnson

W. H. Tyree

Exxon Nuclear Co. Fuel Fabrication Plants:

W. S. Nechodom

Kerr-McGee Nuclear Corporation, Cimarron Facility:

G. J. Sinke 
-vii-

TABLE OF CONTENTS

1. Introduction, Recommendations, and Summary . . . . . . . . . . . 1

1.1 Introduction . . . . . . . . . . . . . . . . . . . 1

1.2 Possible Improvements in Monitoring Instrumentation, Systems,

and Programs . . . . . . . . . . . . . . . . . 2

1.2.1 Instrumentation . . . . . . . . . . . . . . . 2

1.2.2 Data Handling Systems . . . . . . . . . . . . . 5

1.2.3 Monitoring Programs. . . . . . . . . . . . . . 6

1.3 Summary . . . . . . . . . . . . . . . . . . . 7

2. Nuclear Facilities, Release Possibilities, Standards, and

Monitoring Objectives. . . . . . . . . . . . . . . . 10

2.1 Facilities . . . . . . . . . . . . . . . . . . . 10

2.2 Release Points. . . . . . . . . . . . . . . . . . 19

2.3 Standards . . . . . . . . . . . . . . . . . 22

2.4 Monitoring Objectives. . . . . . . . . . . . . . . . 28

3. Techniques for Identification of Uranium and Plutonium. . . . . . . 34

3.1 Chemical and Isotopic Composition. . . . . . . . . . . . 34

3.2 Radioactive Decay Monitoring . . . . . . . . . . . 38

3.2.1 Air Monitoring . . . . . . . . . . . . . . . 42

3.2.1.1 Alpha Spectroscopy . . . . . . . . . . . . 43

3.2.1.2 Lifetime Analysis. . . . . . . . . . . . 49

3.2.1.3 Associated $\alpha-\beta$ Measurements . . . . . . . . 52

3.2.1.4 Radiochemical Analysis and High-Volume

Air Sampling . . . . . . . . . . . 53

3.2.2 Radiochemical Analysis of Water, Soil, and .

Biological Samples . . . ; . . . . . . . . . . 54

3.2.3 Methods for Field Survey and Personnel Monitoring . . . . 59

3.2.4 Track-Etch Technique . . . . . . . . . . . . . 67

3.3 Monitoring via Induced Activities . . . . . . . . . . 667 67

3.3.1 Neutron-Induced Fission . . . . . . . . . . . . 68

3.3.1.1 Delayed Neutrons . . . . . . . . . . . . 68

3.3.1.2 Fission Track-Etch Techniques . . . . . . . . 69

3.3 .2 Neutron Activation Analysis . . . . . . . . . . . 69

3.3 .3 X-ray Fluorescence . . . . . . . . . . . . . . . 69

3.4 Monitoring Using Mass Spectroscopy . . . . . . . . . . . 71

3.5 Other Monitoring Techniques . . . . . . . . . . . . . 72

3.6 Associated Radiochemical and Physical Techniques. . . . . . 74 
3.6.1 Chemistry . . . . . . . . . . . . . . . . 74

3.6.2 Size and Collection. . • . • . • . . . . . . . 74

4. Current Practice : Monitoring Instrumentation, Programs,

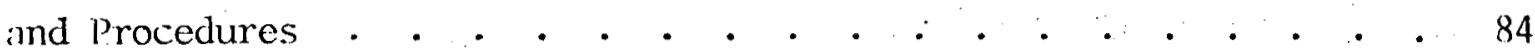

4.1 Techniques: Availability, Cost, and Extent of Application. . . 84

4.2 Release Monitoring at Commercial and Federal Nuclear

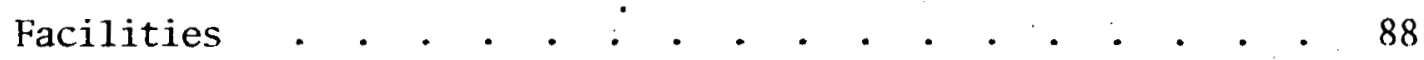

4.3 Procedural and System Aspects of a Monitoring Program. . . . 90

Glossary . • • • • • • • • • • • • • • • • • • • . .93

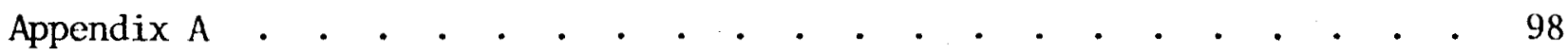


1. INTRODUCTION, RECOMMENDATTIONS, AND SUMMARY

\subsection{Introduction}

Uranium and plutonium, the two basic elements of both commercial and military nuclear power, are both recognized to pose important radiological dangers to man. Accordingly, standards have been established defining limits both to the amount of these materials that may be ingested by humans, and - considering the specific pathways for uptake - to the amount which may be present in various environmental media. It is important that means be available for monitoring both the amounts of these materials that actually escape into the environment and the concentrations in which they are present in any environmental medium. The purpose of this report is to summarize the state-of-the-art of instrumentation for monitoring release of uranium and plutonium into the environment, to compare the capabilities of this instrumentation with the needs as specified by current standards, and to discuss possible ways to improve measurement capabilities. We discuss the types of instrumentation that are currently employed at nuclear facilities, those that are planned for new facilities, developments which are now taking place in laboratory instrumentation, and techniques which might suitably be investigated. For each type of instrumentation, we discuss how instrumental sensitivity and versatility compares with the requirements imposed by current limitations on releases and concentrations. Recommendations are made for improvements in instances where present instrumentation appears inadequate to meet current needs.

It should be noted that this discussion does not treat instrumentation for monitoring of releases other than uranium and plutonium. While releases of other radionuclides may, in many cases, exceed those of uranium and plutonium, they are beyond the scope of this work.

The next section, 1.2, discusses possible improvements in monitoring methods. The basis for these comments is presented in the main body of the report: section 2 constitutes an introduction to nuclear facilities and related standards for uranium and plutonium; section 3 treats actual monitoring techniques, relating them to the objectives outlined in section 2 ; section 4 describes the current availability of these techiniques and the extent of their application at existing facilities. A brief summary of the report and recommendations is given in section 1.3 . 
1.2 Possible improvements in monitoring instrumentation, systeiss, and programs

Techniques for monitoring uranium and plutonium are presented in section 3 . With few exceptions, the basic physical characteristics of such instrumentation are relatively well-defined. This is also the situation for the more general class of instrumentation for monitoring all types of environmental radiation and radionuclides. Largely because of this stabilization in the understanding of fundamental monitoring techniques, there is a consensus that the aspects of monitoring programs that are most susceptible to improvement are not the basic detection and analysis instrumentation, but rather the definition of program objectives and the manner in which data are handled. This same attitude is app $1 \mathbf{i -}$ cable to the case of uranium and plutonium monitoring. Although a number of pos sible improvements are suggested in section 3 and stated explicitly in this section, it is still true that a common feature of all current instrumentation is that many hours are required, either in sample collection or sample analysis, to identify concentrations at the level of the maximum permissible concentration for the general public. Therefore, in addition to possible improvements in sensitivity due to the instrumental techniques, we point out (in section 4.3) that improvements may be made in data handling and in program delinition. In the present section, we identify specific possibilities for improvement in monitoring instruments themselves, then proceed to discuss corresponding possibilities in system and program aspects of monitoring.

\subsubsection{Instrumentation}

The most important class of instruments for monitoring of uranium and plutonium includes those devices which sample air, then detect alpha radiation from radionuclide decay (section 3.2 ). The sensitivity of these may be improved in principle by making the instruments larger. Such a "brute force" approach to improving sensitivity would be accomplished by: 1) raising the air sampling rate by incorporating larger pumps, and 2) altering the detection arrangement to take advantage of the resulting higher sampling rate. This second alteration would require the use of larger detectors or detector arrays to monitor the larger filter areas needed for high sampling rates. This combination of higher sampling rates with larger detectors could improve sensitivities by an order of magnitude, without taking the approach of current high-volume samplers (section 3.2.1.4), which use radiochemical analysis prior to alpha spectroscopy.

In addition to increasing the effective sampling rate of alpha monitoring instruments, changes can be made to permit use of the more sophisticated data 
analysis methods discussed in section 1.2.2. For an alpha monitor, the two inprovements of greatest potential are 1) to improve the detection energy resolution and 2) to make use of lifetime analysis to discriminate against radondaughter backgrounds; implementation of corresponding analysis techniques are discussed in 1.2.2. For any continuous monitor to make these improvements, the physical arrangements for sampling and detection would have to be changed. High-resolution alpha spectroscopy requires a counting chamber that is at least partially evacuated to avoid alpha energy degradation in the air in the chamber. Lifetime analysis requires an off-line counting arrangement, so that the time dependence of counting rates can be analyzed. One scheme which would satisfy both of these requirements is to use a continuous filter strip and, at regular time intervals, step the portion on which particles have just been collected into a partially evacuated counting chamber, where a detector can monitor the alpha decay rate, which can then be subjected to analysis combining alpha spectroscopy and 1ifetime analysis. Such a detector and analysis arrangement i,s presently being developed at Lawrence Livermore Laboratory.

Such improvements in analysis techniques can also be extended to instruments other than alpha monitors. For example, one of the standard instruments for area surveys for plutonium had been the FIDLER detector (section 3.2.3), which uses a single thin crystal as a detector. On the other hand, the Phoswich detector (also in section 3.2.3), employed for plutonium assay in human subjects, has heightened sensitivity due to the fact that a second detector is placed behind the first to eliminate background due to partial absorption in the first detector. Presumably because of the more delicate physical assembly and electronics required for such analysis, this technique has not often been applied to field survey instruments. However, there have been substantial improvements in recent years in the ability to incorporate complex electronic analysis into relatively sma11, stable packages - as discussed in section 1.2.2 - and changes in instrument design which take advantage of such improvements can make a substantial difference in the sensitivity of, not only alpha monitors, but also survey and environmental instrumentation.

Most alpha monitoring instrumentation does not take specific account of the particulate nature of uranium and plutonium activity, except in the one respect that filter collection does depend directly on particularity. As discussed in section 3.6, particle sizes affect not only the biological significance of radioactivity, but also the effectiveness of monitoring techniques. There are two distinct respects in which a technique may take advantage of the size 
distribution of the uranium and plutonium bearing particles. The first is based on the fact that a large percentage of the particles may be segregated into a separate fraction by impaction, either actual impaction onto a surface or virtual impaction into an air stream that is substantially sma11er than the total air stream drawn for sampling. Handling a small air stream that still contains most of the activity of interest is a substantial advantage in many monitoring techniques; it inplies that smaller filter areas may be used and that these may be monitored by smaller detectors than in the situation where the entire sampled air stream must be handled. Such a virtual impaction technique could be applied in many situations including, for example, high volume sampling with alpha monitoring and the mass spectrographic techniques now being developed (section 3.4). The possibility of developing virtual impaction techniques applicable to uranium and plutonium monitoring should be investigated.

As discussed in section 3.6, other impaction techniques have already been applied in isolated cases, primárily to segregate the particles which carry most of the radon-daughter background. This is a second respect in which segregation by impaction may improve monitoring sensitivity. It is important to note that the application of virtual impaction as described in the previous paragraph to any monitoring technique automatically segregates against very sma11 particles, the ones which carry a larger share of radon daughters than of uranium and plutonium. As a result, incorporation of a virtual impaction stage not only decreases the air stream to be monitored, but may decrease the relative amount of radon daughters present as background in the detection stage.

The above possibilities for improvement apply not only to source, but also to environmental air monitoring. Air monitoring in the field typically depends on high-volume sampling, followed by radiochemistry and alpha spectroscopy. However, the improved sensitivity available from some combination of larger sampler-detectors, better data analysis, and virtual impaction segregation may make field samplers with continuous readout capabilities a practical approach. The disadvantage to incorporating such techniques into field units is that these techniques involve greater cost and complexity than a simple high-volume sampler. However, the time delay and cost of radiochemical laboratory techniques would be avoided.

Fina11y, we should point out the largely unexplored potential of techniques that do not involve detection of decay radiation. One such technique is mass spectroscopy, particularly that which uses a direct inlet ion source for air 
monitoring applications. Development of the direct inlet method should be continued, and - in this connection - the possibility of using virtual impaction to increase the sampling rate should be investigated.

Another technique for which the intrinsic analysis time is relatively small is $x$-ray fluorescence. The possibility of optimizing this technique for heavy radionuclides, particularly those with low specific activity is worthy of investigation.

Both direct-inlet mass spectroscopy and $\mathrm{x}$-ray fluorescence are applied primarily to on-line air monitoring situations. In modified form, they have broader application. Attention should be given to possibilities for rapid introduction of various types of samples into mass spectrographic or $x$-ray fluorescence analyzers. For example, a mass spectrograph can greatly shorten the time that would otherwise be taken for an alpha spectrometer to make a measurement on a sample prepared by radiochemical separation.

\subsubsection{Data Handling Systems}

It is now generally possible to improve radiation instrumentation by complementing the radiation detector itself with a compact electronic package that is a preprogrammed data analyzer and instrument controller. Such a package, based for example on microprocessor technology, provides the potential for more sophisticated analysis of data from the detector and for more complex contro1 of the detector and of derivative information. We give a number of important examples:

1. A simple alpha spectroscopic unit, with radon-daughter compensation, sets two windows on the output of the amplified signal from the detector and subtracts the counts in one window from those in the other, with some weighting factor. A more sophisticated analysis would store al1 the energy information in a region of interest, and would perform a fit, with some peak-shape, to the accumulated spectrum. This would give a more accurate result for the number of counts due to the activity of interest.

2. Analysis such as that above would yie1d, in a systematic way, more information than simply the count rate. It would also yield the uncertainty in that result. Most on-1ine monitors cannot provide this information, and hence are more susceptible to false alarms and misinterpretation due to the introduction of uncertainties, for example, that arise from subtraction of two large count rates to obtain a smaller net rate. 
3. If the controller causes the counts from the detector to be stored in sequential time bins, the resulting information can then be used to perform lifetime analysis to obtain the total long-lived count rate, part of which would be attributable to uranium and plutonium. (If desired, this could then be combined with spectroscopic analysis, as above, to yield an even more precise result.) This would assume, of course, that the sample was being monitorcd after collection of the sample had stopped. This could be arranged, as discussed in section 1.2.1, by using a strip filter, which moves at intervals so that the portion most recently used for collection is moved to the counting position.

4. In situations where lifetime analysis per se is not being performed, the results can still be stored at regular time intervals. Thus, for exarmle, if the accumulated number of counts exceeds a preset level, the device will have stored more detailed time information, so that personne1 can te11 whether or not the indicated release has just occurred.

In each of the examples just listed, the function indicated required some combination of control, storage, and analysis, all of which can be performed by the small, inexpensive, easily progranmed microprocessors now available. In many monitoring applications, use of this technology should be encouraged.

As discussed in section 4.3 , there are additional levels at which data handling and/or analysis occurs, beyond the level of the instrument itself. Whether for data transfer (from monitoring location to a readout-control point), for comparison with standards, for examination for possible ameliatory action, or simply for incorporation into summary reports, efficient and dependable methods improve the effectiveness of a facility monitoring system. In many instances, these processes, also, are most suitably based on the minicomputer and microprocessor technology that has recently become available.

\subsubsection{Monitoring Programs}

Although the primary concern of this work has been the design of instruments themselves, the overall effectiveness of monitoring systems is directly affected not only by instrumental design, but a1so by more general aspects of the program design. The most basic of these aspects is the actual development of radiation standards, on which we will not conment. Other aspects which involve more specifically the monitoring instrumentation itself are the following:

1. Just as there is a central source of information on radiation standards, it would be helpful were there a similar store of information on available instrumentation, the manner in which it can be incorporated into systems, and how 
one finds or trains personnel qualified to implement and operate these systems. To a limited extent, the Survey of Instrumentation for Environmental Monitoring (Ref. 1) performs this function. However, such a function should be broader, so that it provides more detailed information and, more importantly, so that the designer of a monitoring system is aware that this information exists, just as he is aware of the radiation standard with which instrumental results will be compared.

2. The centralized administration of standards, whether it is the Nuclear. Regulatory Commission, the Fnvirommental Protection Agency, or the National. Bureau of Standards could also provide information on the most effective means for monitoring associated with any facilities that are being 1 icensed. Alternatively, it could refer licensees to the central information source mentioned above.

3. For use in emergencies, regulatory agencies could have available special instrumentation for use in the areas of concern. 'This instrumentation might, for example, be housed in a mobile van which could be flown into the exposed area on short notice. The instrumentation could be of a more specialized and/or sensitive nature than that likely to be available at the typical commercial nuclear facility. Measurement results could be used for determining immediate courses of action in population protection, and aiso for estimating the long-term effects of the release. Availability of specialized instrumentation from a central source would enable individual facilities to devote their monitoring programs to the specific task of source monitoring, a function which more directly contributes to the detection and control of abnormalities. On the other hand, central source availability would, to a limited extent, relieve the pressure on the regulatory agencies to have specialized environmental monitoring instrumentation available in the vicinity of every nuclear facility.

Each of these comments on monitoring programs, as opposed to instrumentation or systems, applies as well to the general case of radiation monitoring as to uranium and plutonium monitoring. The possibilities for improvement that apply specifically to uranium and plutonium monitoring are discussed above in section 1.2 .1 and in 1.2.2. As noted there, although the most important features of instrumentation for monitoring of uranium and plutonium are relatively we11 determined, there are substantial opportunities for improvement.

1.3. Summary

We have examined the techniques now available for monitoring of uranium and plutonium releases, including those based on detection of decay radiation 
and those utilizing other characteristics of these elements. Means do exist for monitoring at the various types of fuel cycle facilities. However, in many facilities rather rudimentary monitoring techniques are employed and, moreover, for some applications, the sensitivity and versatility of instruments currently available preclude rapid identification of releases, at least as compared with maximum permissible concentrations. There is potential for improvement of instrumental sensitivity and also for assurance that appropriate monitoring systems are employed at each type of nuclear facility. Accordingly, we have indicated that the following possibilities for improvement should be investigated: Instrumentation:

-design of larger alpha monitoring instruments with greater effective sampling rates

-change of alpha monitoring instrumentation design to permit more sophisticated data analysis, including partially evacuated counting chambers, permitting high resolution spectroscopy, and continuous filter strips, stepped to allow lifetime analysis

-redesign of survey instruments to take advantage of more sophisticated ana1ysis techniques

-use of virtual impaction to reduce the total air stream sampled to a smaller stream for actual handling in the detector system

-use of impaction techniques to discriminate against particle sizes which carry a disproportionate share of background activities

-development of direct inlet mass spectroscopy, and examination of the value of using virtual impaction segregation prior to the inlet -optimization of $\mathrm{x}$-ray fluorescence for detection of uranium Data handling systems

Encourage design of compact electronic packages for sophisticated control of instruments and subsequent data analysis. Specific possibilities:

-improved analysis of alpha energy spectra

-more precise readout. of uncertainties in any stated result

-convenient lifetime analysis

-simple sequential storage of data for subsequent analysis

The same microprocessors which supply these capabilities can improve the general. structure of a facility monitoring system. 
Programs

-creation of a central source for detailed information on instrumentation -regulatory recommendation of techniques suitable for specific facilities -rapid availability of specialized monitoring equipment for emergency use Some of these possibilities are now being investigated, while others are not. Pursuit of these various possibilities will lead to improvement in instrumenta1 capabiities and in the effectiveness of monitoring programs.

REFERENCES for Section 1

1. Survey of Instrumentation for Environmental Monitoring, Lawrence Berkeley Laboratory Report LBL-1. 
2. NUCLEAR FACILITIES, RELFASE POSSIBILITIES, STANDARDS, AND MONITORING OBJECTIVVS 2.1 Facilities

A summary of the principal facilities involved in production of commercial nuclear electric power is given schematically in Fig, 2-1. These facilities perform a long sequence of operations both for production of fresh fue 1 (the "front" half of the nuclear fuel cycle) and for treatment of irradiated fucl (the "back" end of the fuel cycle). In front of the nuclear reactor are the facilities for mining and milling of uranium ore, conversion to uranium hexafluoride, enrichment of the uranium, and fabrication of the fuel elements; all of these facilities are involved in the procurement and processing of nuclear materials to produce the actual fuel that is inserted into the reactor core. Following the reactor are the facilities that handle irradiated fuel, reprocess the fuel elements to extract the remaining useful nuclear materials and also the radioactive wastes, and redirect the nuclear materials for recycle (or storage) and the wastes for storage (or disposal). The principal facilities on the "back" end of the fuel cycle are not now in operation; their general nature, however, is rather well understood. On the other hand, the fuel production facilities were established long ago, since generation of nuclear power is predicated on their operation. As a result, a large amount of experience has been amassed in the commercial handling of uranium fue1s; as yet, the same is not true for plutonium.

Production facilities begin with the mining and milling operations which extract uranium ores from the earth and process them to yield a product which consists of 70 to $90 \%$ uranium, the chemical concentrate on which subsequent operations are typically based. Since uranium ores in the United States usually contain less than $1 \%$ uranium, substantial processing is required. This is usually accomplished at a mill situated close to the mine. Although it is possible to achieve some separation by physical means, the basic scheme is to crush the ore, then leach out the uranium by mixing the crushed ore with an acidic or alkaline solution (sulfuric acid is often used). The uranium that goes into solution is then extracted by a sequence of chemical operations (such as ion exchange or solvent extraction, followed by precipitation and drying). This process leaves the uranium in the form of $\mathrm{U}_{3} \mathrm{O}_{8}$ concentrate of the required purity. An important 
$=$

₹

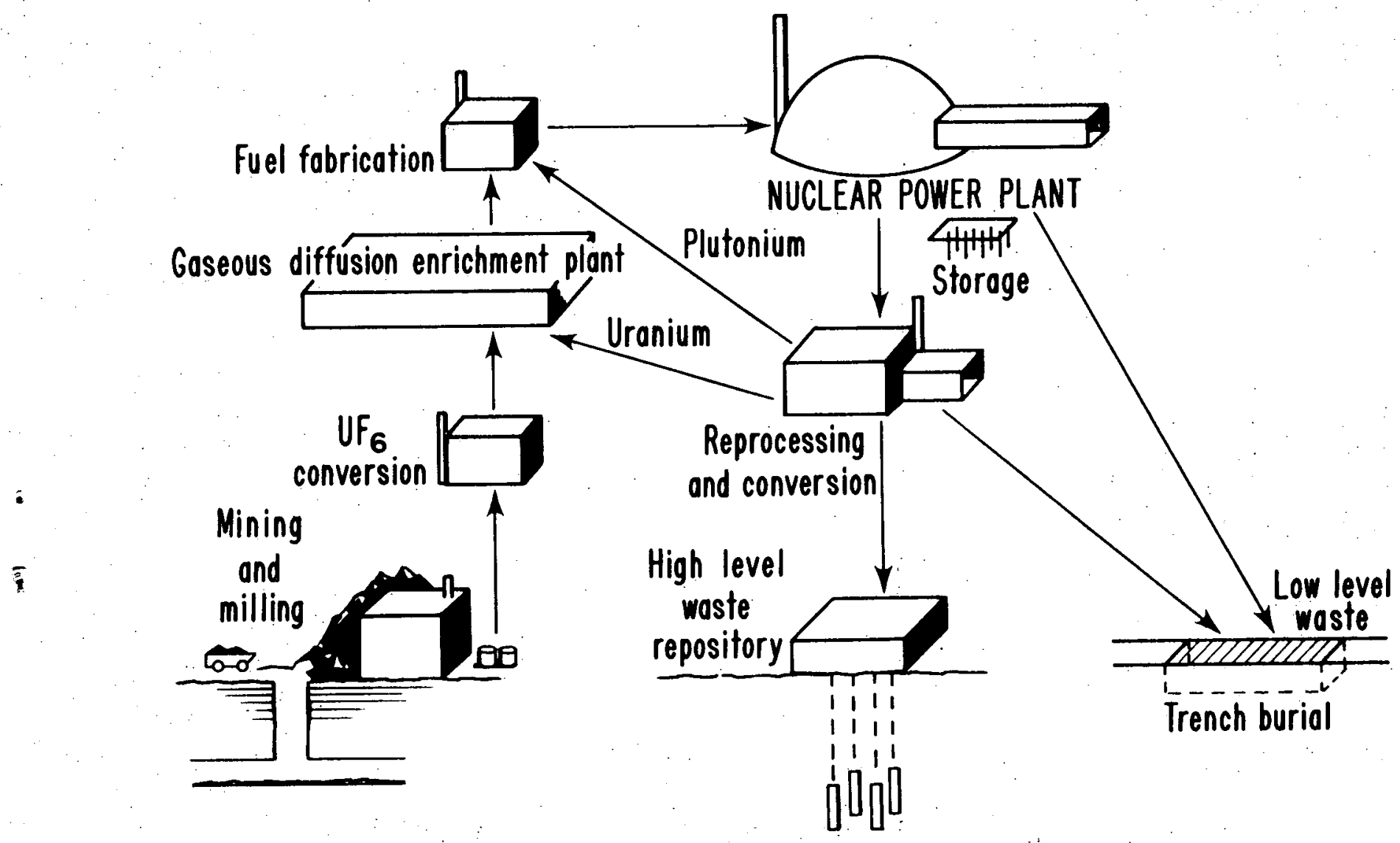

NUCLEAR. FUEL CYCLE

XBL $768-3258$

Fig. 2-1. Major facilities of the nuclear fuel cycle. 
remant of the milling operation is a "tailings" pile, containing the ore material out of which the uranium was extracted, Both this pile and the mine and mill themselves may be sources of significant radiation exposures of select population groups, principally workers at the facilities themselves. However, the doses received are usually associated with radon daughters rather than with uranium itself.

The uranium concentrate is transported in drums to a $\mathrm{UF}_{6}$ conversion plant. There it is necessary both to purify the uranium and to convert it to uranium hexafluoride, the compound which is processed at the isotopic enrichment plant, It is particularly important to remove any impurities that would reduce the quality of the uranium as a nuclear fue1, especially those nuclides with large neutron-absorption cross sections. This purification is achieved by an extraction process (similar to that used at the mil1), the product of which is oxidized to $\mathrm{UO}_{3}$. The $\mathrm{UO}_{3}$, in turn, is converted by treatment with hydrogen, hydrogen fluoride, and fluorine to uranium hexafluoride $\left(\mathrm{UF}_{6}\right)$, a solid at room temperature. The $\mathrm{UF}_{6}$ is shipped to gaseous diffusion plants for enrichment. Thus the principal operations at the conversion plant are chemical in nature. The main constituent of the process stream in this plant, and in the remaining frontend facilities, is relatively pure uranium, in one chemical form or another. Natural uranium has an isotopic makeup of $99.3 \%{ }^{238} \mathrm{U}$ and $0.7 \%{ }^{235} \mathrm{U}$. The ${ }^{235} \mathrm{U}$ is "fissile" (i.e., it can be induced to fission by therma1 - or slow -neutrons) and is thus the isotope of primary interest in thermal reactors, such as the light-water reactor (LWR), the reactor on which commercial nuclear power is currently based. LWR's require that the ${ }^{235} \mathrm{U}$ concentration in the fresh fuel be increased from the natural $0.7 \%$ to about $3 \%$ by some isotopic enrichment process.

Enrichment plants in the United States currently employ a gaseous diffusion method. In gaseous diffusion, use is made of the fact that $\mathrm{UF}_{6}$ con- $^{-}$ taining ${ }^{235} \mathrm{U}$ (the fissile isotope) has a lower molecular weight than that containing ${ }^{238} \mathrm{U}$. When heated to a gaseous form, the lighter molecule collides more often with container walls and therefore will diffuse through a porous barrier slightly faster than the heavier. The enrichment plant consists of a large number of such barriers in a "cascade". where, at any given stage, the 
enriched product proceeds to the next higher stage, while the depleted remnant is directed to the next lower. In principle, a cascade can be designed with enough stages to yield a product of arbitrary ${ }^{235} \mathrm{U}$ purity. In practice, the highest enrichment uranium for commercial purposes would be used in the high-temperature gascooled reactor (HTGR), which requires uranium feed that is $93 \% 235 \mathrm{U}$, much higher than the 2 to $4 \%$ enrichment for LWR's. Other methods of enrichment are currently at various stages of development. A method that is employed in new European enrichment facilities uses high-speed centrifuges, which again depend on differences in the molecular weight of compounds containing ${ }^{235_{U}}$ and ${ }^{238} \mathrm{U}$ to provide the isotopic separation. In thịs method, it is also necessary to employ a series of stages, although the number is not as large as in a diffusion plant. It is likely that any privately built enrichment facility in the United States would employ the centrifuge method, due to economic advantages. Finally, a technique that is receiving a great deal of attention currently and toward which much research is being directed is the "laser separation" method. This depends on the principle that the energy (or, equivalently, the wavelength) of spectral lines of uranium or its compounds will depend slightly on which uranium isotope is present. Using lasers of very well-defined wavelength, it should be possible to induce transitions in one isotope, but not the other, thus leading to a separation. Although the details of this method are not yet developed, it is clear that the process stream would involve radically different types of operations than are employed in current separation methods. Choice of laser separation methods would result in an altered potential for radioactive releasès.

Uranium hexafluoride of the desired enrichment is shipped to fuel fabrication plants, where uranium is incorporated into assemblies ready for insertion into a reactor. A typical process line would expose the UF ${ }_{6}$ to ammonium hydroxide, yielding ammonium diurynate, which is dried, then reduced (using hydrogen) to $\mathrm{UO}_{2}$, the chemical compound actually used as fuel. The $\mathrm{UO}_{2}$ is ground into a powder which, for water-cooled reactors, is pressed into pellets, sintered (heated) to form a ceramic, then finally ground to a cylindrical shape. Thesecylindrical pellets are incorporated into fuel rods (pins), with jackets (cladding) of zirconium alloy. The fuel rods are arranged in assemblies ready for insertion into the reactor core.

One of the materials extracted at the fuel reprocessing plant (see below) is the plutonium produced during reactor operation. This plutonium may then 
serve as fuel material for fresh fuel assemblies. Any plutonium incorporated into the fuel would be subject to a fabrication process similar to that just described for uranium. As presently planned, when plutonium is recycled, it would be added as $\mathrm{PuO}_{2}$ powder to the $\mathrm{UO}_{2}$ just mentioned. As indicated, the fabrication process for either uranium or plutonium involves both chemical and physical operations, each with characteristic release possibilities.

In light-water reactors in the United States, fuel remains in the reactor for three or four years of operation, one third or one fourth of the fucl being replaced each year. During reactor operation, the total amount of radioactivity in the fuel increases dramatically (as measured in curies). Although almost a11 of this increase is associated with fission products, the total amounts of uranium and plutonium activity increases substantially, principally as a result of neutron capture. On removal from the reactor, the fuel is stored for several months in water pools, during which time the fuel's overall radioactivity decreases markedly, due to decay of short-lived species. However, rapid decay does not occur, for the most part, for the isotopes of uranium and plutonium. The fuel, cooled and shielded by special shipping casks, would then be transferred to a fuel reprocessing plant, if such facilities were available.

At the reprocessing plant, the fuel rods are chopped up and placed in nitric. acid to dissolve the fuel. The cladding hulls are relegated to waste storage, leaving an acidic solution from which the uranium and plutonium are removed by solvent extraction. The remnants are the "high-level wastes", which consist primarily of fission products in acid solution. The plutonium may then be separated from the uranium by another solvent extraction, and the uranium purified by processes similar to those at the $\mathrm{UF}_{6}$ conversion plant. In the extraction of uranium and plutonium from the acid solution, care is taken that all of the highly radioactive fission products remain in the wastes. As a result, the extraction process leaves a smal1 portion of the uranium and plutonium - perhaps $1 \%$ - in the highlevel waste fluid.

A full-scale fuel reprocessing plant, to be operated by Allied-General Nuclear Services, is now nearing completion at Barnwe11, South Carolina. This plant will be able to process 1500 metric tons of heavy metal (uranium and products) per year, equal to the output of about $50 \mathrm{LWR}$ 's. * A major question *It is anticipated that other fuel processing plants will be established by the middle of the $1980^{\prime} \mathrm{s}$. One would be the Nuclear Fue1 Services plant at West Valley, New York; this plant closed down in 1972 for an extensive renovation intended to reduce releases and occupational exposures and to bring the plant up to a 750 metric ton per year capacity. In addition, Exxon is now considering the possibility of building a plant. 
in the licensing of this plant will be uncertainty in the handling of plutonium. In a complete LWR fuel cycle, the uranium would be returned to enrichment plants, the plutonium to fuel fabrication plants.

The high-level wastes are an acidic, highly radioactive solution which could be partially consolidated by evaporation, but which must ultimately be reduced to a solid in any case. Methods are available for "calcining" these wastes to a dry, granular solid which can be stored on an interim basis in stainless steel bins or capsules. However, the ultimate disposal form that is most favored is a silicate glass that would be deposited in geologically inactive formations. Although the details of such disposal are not settled, methods for transformation to glass are known. Both calcining and glassifying require raising the temperature of the liquid wastes to drive off volatile components. The less volatile residue is ultimately allowed to $\operatorname{coo} 1$ to a solid. In the case of glassification, glass-forming materials are first added to the residue and the temperature is raised sufficiently that, on cooling, the melt forms the desired type of glass. The volatile substances driven off constitute a secondary waste stream which must be handled separately or, to some extent, can be reinjected at the beginning of the solidification process. The final product of glassification is a hard but brittle substance, with a tolerably low thermal conductivity (it would be an improvement if it were higher) and very low leachability. Such a product is intended to be suitable for permanent disposal. As indicated above, the process is not ideal since it requires driving off substantial amounts of liquid, which may contain significant radioactivity. However, as previously noted, the high-level wastes do not contain a large portion of the uranium or plutonium from a reactor, even though it is still enough to warrant monitoring.

We have not commented on the relatively inert phases of the fuel cycle, such as storage or transportation, since these do not involve processes which would tend to produce appreciable effluent. There is however, a potential for release under accident conditions, or even under the relatively passive situation in which, due to some failure, the cooling necessary to maintain the integrity of the radioactive material is not supplied.

The facilities indicated in Fig. 2-1 are those necessary for production and reprocessing of fuel for a light-water reactor system. A general discussion of light-water reactors and related facilities and their radiological impact is contained in Refs. 1 and 2. Some obvious changes in this fuel cycle would be necessary were breeder reactors, or even thorium-based reactors, to come into use. A breeder reactor system as presently envisioned would differ from the LWR 
fuel cycle in one striking way; because the fissile material is primarily plutonium, and the isotopic composition of reactor-produced plutonium is adequate for recycle, no enrichment of the fuel is necessary. Uranium would still be used as the fertile material (to be converted to plutonium), but the mass 238 isotope is the one required. Enrichment plants, in the process of producing LWR fuel enriched in $235^{U}$, are storing the remnant (or "tails"), i.e., uranium that is depleted in ${ }^{235} \mathrm{U}$, so that its ${ }^{238} \mathrm{U}$ content is even higher than the natural 99.3\%. These enrichment tails would be used in breeders for a substantial period after their introduction, so that all of the fuel supplied externally to the breeder fuel cycle would effectively be LWR byproducts.

In this context, it is appropriate to note that light-water reactors are examples of "converter" reactors, i.e., as fissile nuclei are destroyed in the course of the nuclear chain reaction, some fraction of them is replaced by fertile nuclei which "convert" to fissile by capturing neutrons. (In a breeder reactor, more fissile material is produced than is destroyed.) Thermal reactors may be designed with a high conversion efficiency by using thorium 232 as the fertile material instead of ${ }^{238_{U}}$. (See Ref. 3 for further discussion of both converter and breeder reactors.)

Use of converter reactors where the initial fuel material contains relatively pure ${ }^{235} \mathrm{U}$ as the fissile nuclide and thorium as the "fertile" nuclide would also imply alteration of the fuel cycle. (Examples of such reactors would be the high-temperature gas-cooled reactor or the proposed light-water breeder. reactor.) Eventually, as the fuel cycle reaches equilibrium, most of the fissile material supplied to the reactor is ${ }^{233} \mathrm{U}$ generated from thorium; this uranium does not require enrichment since it is not diluted by ${ }^{238} \mathrm{U}$. Thus ${ }^{233} \mathrm{U}$ effectively replaces plutonium as the latter would be used in the LWR or breeder fuel cycle. However, any ${ }^{235_{U}}$ that is supplied must be more highly enriched than in the LWR cycle. An auxiliary mining and milling function would have to be established to supply thorium to the fabrication plant, and thorium recovered from reprocessing would be recycled after holdup to allow certain species to decay.

Recycled uranium that is generated from 232 Th would contain two isotopes. that require more attention than ${ }^{235} \mathrm{U}$ or ${ }^{238} \mathrm{U}$, the naturally occurring isotopes: 1) a smal1 portion (about $0.1 \%$ by mass) of the generated uranium is $232 \mathrm{U}$, which has a short half-life, and therefore a high specific activity. Further, because its daughters are short-lived, they have to be considered in setting 


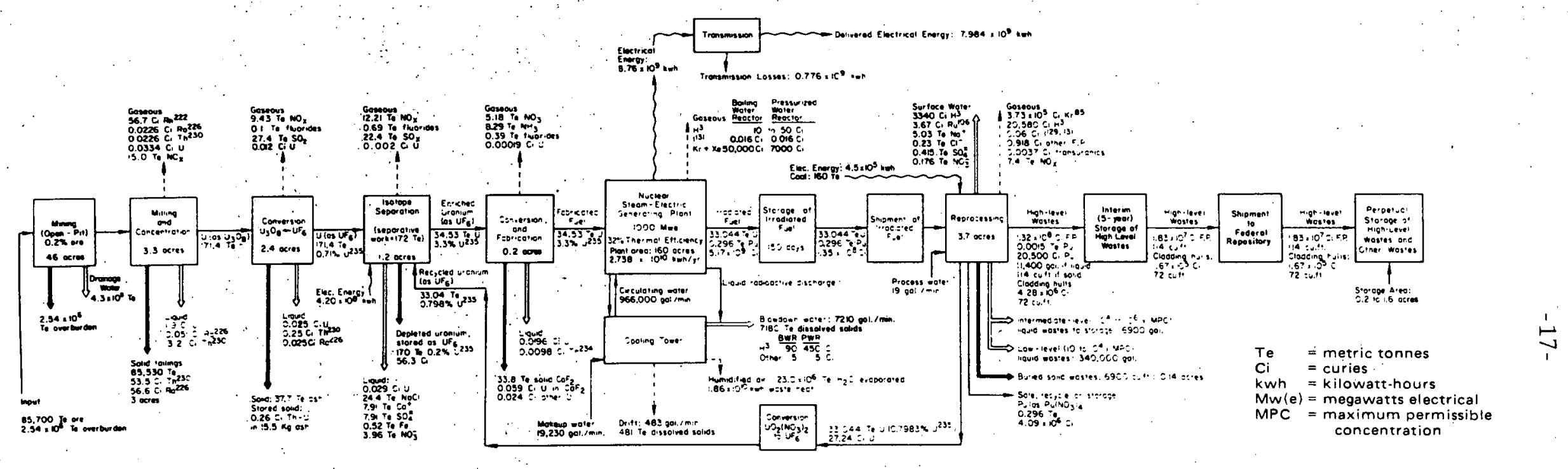

Fig. 2-2. Material and environmental release flow sheet for a typical 1000 M. We light water reactor power plant. 


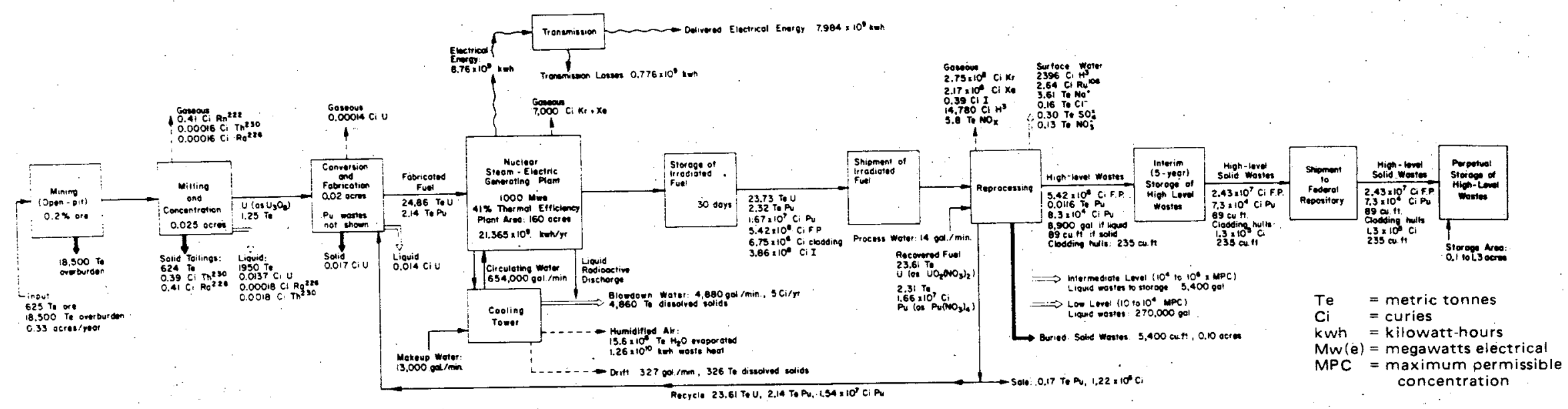

Fig. 2-3. Material and environmental release flow sheet for a 1000 Mwe breeder reactor power plant. 
standards. Even more important - for some purposes - is the fact that the decay of ${ }^{208} \mathrm{Tl}$, one of the daughters, is accompanied by emission of a $2.6 \mathrm{MeV}$ gamma ray. This is much more penetrating than the radiation from other important uranium and plutonium isotopes, so that additional shielding and remote handling equipment would have to be added in recycle facilities to accommodate this isotope. 2) The primary isotope to be recycled, ${ }^{233} \mathrm{U}$, has a decay mode, halflife, and lung-clearance rate (for any insoluble form) that is similar to ${ }^{239} \mathrm{Pu}$. As a result, permitted insoluble concentrations by either mass or activity are not dissimilar from those of ${ }^{239} \mathrm{Pu}$ (see section 2.3).

2.2. Release Points

In the light-water reactor fuel cycle, routine radioactive releases consist primarily of gaseous fission products (noble gases and tritium) which are freed from the fuel principally at the reprocessing plant. ${ }^{4}$ Significant relcases also occur at the reactor site itself. In addition, notable releases in the form of ${ }^{226} \mathrm{Ra}$ and its daughters occur at the mill site. These are indicated in Fig. 2-2, a flow sheet for the LWR fuel cycle (without plutonium recycle). Note that the amounts given in the figure are in curies, a measure of activity, rather than in any toxicity index. A comparable fuel cycle flow sheet is given for the liquid metal fast breeder reactor (LMFBR) in Fig. 2-3.

The potential for release of uranium and plutonium is rather small throughout any of these fuel cycles, except under accident conditions. One possible exception is at the mill site, where the tailings will necessarily contain substantial quantities of uranium, i.e., the portion missed by the extraction process. To some extent, particles from these tails will become resuspended in the air by errosive action, resulting in measurable concentrations in air; liquid effluent streams will also contain uranium: In other parts of the fuel cycle, the quantities of process material are smaller, and their form more uniform, so that greater control can be exercised; as a result, ordinary concentrations of uranium and plutonium in the effluent stream can be kept to levels that are within regulatory limits.

*From Fig. 2-2, the total amount of uranium routinely release in gaseous and liquid waste streams from $\mathrm{UF}_{6}$ conversion, isotopic enrichment; and fuel fabrication, sums to roughly 0.1 curies per reactor year of operation, of which about $90 \%$ is in the liquid effluents. Another $0.1 \mathrm{Ci}$ is released in the milling operations. Much smaller amounts are released from the reactor and subsequent facilities, where the transuranic releases (including plutonium) of $0.003 \mathrm{Ci}$ exceed the uranium releases. (Total activity releases of other nuclides, principally noble gas fission products, are much greater than uranium or plutonium releases.) 
As noted, the most significant releases of these materials may occur during irregularities in the operation of nuclear facilities, rather than under routine conditions. Some historical examples of releases from plutonium facilities are given in Table $2-1^{*}$ (from Ref, 5) and in Ref. 6 . It is difficult to draw general conclusions from these examples of accidential release, since the particular facility involved, the process occurring (and thus the form in which the uranium or plutonium is to be found), and the type and degree of the irregularity, will determine both the amount and form of material released. These, in turn, set conditions on the measurement capabilities required for monitoring these releases. The best procedure calls for measurement sensitivities ranging from background levels to the "worst case" level of activity.

The sites where monitoring is needed are relatively well defined. Uranium is the major constituent of the LWR cycle from the mine to the reprocessing plant. Plutonium is produced in the reactor and resides in the fuel until it is extracted at the reprocessing plant. Both the uranium and plutonium may be stored and, assuming they are recycled, returned to the enrichment and fabrication plant, respectively. They will also be present in lesser amounts in the waste stream that begins at the reprocessing plant. When a plutonium-based breeder comes into use, this basic pattern will be changed only in that more plutonium and less uranium will be handled. On the other hand, a thorium-based reactor would introduce material of a different character, i.e., ${ }^{233} \mathrm{U}$, which has a specific activity more similar to ${ }^{239} \mathrm{Pu}$ than to ${ }^{235} \mathrm{U}$. This isotope would be present in the fuel cycle from the fuel fabrication plant to the reprocessing plant (with, as usual, a small amount in the high-level wastes).

The nuclear industry has already had substantial experience in the handling and monitoring of uranium. Many aspects of this experience are summarized in the proceedings of a recent conference on occupational health experience with uranium. ${ }^{7}$ Most of this experience is with the front end of the fuel cycle. In addition, the Nuclear Fuel Services plant has provided one example of a commercial reprocessing plant, and its effluent history has been discussed. ${ }^{8}$ However, its design philosophy differed from that expected in new plants. Additionally, severa1 other reprocessing plants have been operating in government facilities (such as at Idaho Falls, Hanford, and Savannah River). In each processing plant, the effluents of major concern have not been uranium or plutonium, but the capability for monitoring them has been available. (See section 4.) Finally, a

* These are only intended to be exemplary. Similar accidents have occurred since 1970 . 
TABLE 2.1. Major Plutonium Containment Accidents ${ }^{5}$

\begin{tabular}{|c|c|c|c|}
\hline Date & Cacegory & Location & Manner and Amount of Pu Release \\
\hline $11 / 16 / 51$ & $\begin{array}{l}\text { Criticality } \\
\text { Excursion in } \\
\text { Criticality. } \\
\text { Teat }\end{array}$ & $\begin{array}{l}\text { R1chland, } \\
\text { Wash. }\end{array}$ & $\begin{array}{l}\text { Pressure buildup Inside a vessel } \\
\text { containing Pu solution caused the } \\
\text { oolution to spray into room. No } \\
\text { environmental release. }\end{array}$ \\
\hline $12 / 4 / 51$. & F1re & $\begin{array}{l}\text { Hanford, } \\
\text { Waoh: } \\
\text { Research } \\
\text { Factlity }\end{array}$ & <4 g; sone Pu outside of facility. \\
\hline $1 / 55$ & P1re & $\begin{array}{l}\text { GE } \\
\text { Install. } \\
\text { at Hanford, } \\
\text { Wash. }\end{array}$ & No Pu outside of facility. \\
\hline $9 / 55$ & Pixe & $\begin{array}{l}\text { Rocky } \\
\text { Flats } \\
\text { plent, } \\
\text { Col. }\end{array}$ & $\begin{array}{l}2.3 \text { ug in air exhaust; none } \\
\text { decected in environment. }\end{array}$ \\
\hline $9 / 57$ & PIre & $\begin{array}{l}\text { Rocky } \\
\text { Flats } \\
\text { plant, } \\
\text { Col. }\end{array}$ & $\begin{array}{l}\text { Emerging saoke (after the ventila- } \\
\text { tion syetem was shut of }) \text { contained } \\
\text { negligible Pu. } \leq 4 \times 10^{-9} \mathrm{~g} \mathrm{Pu} / \mathrm{g} \text { of } \\
\text { vegetation found in vicinity, but } \\
\text { this is considered below hazardous } \\
\text { levels. }\end{array}$ \\
\hline $3 / 31 / 59$ & $\begin{array}{l}\text { Chentcal } \\
\text { Explosion }\end{array}$ & $\begin{array}{l}\text { Pu Metal } \\
\text { Machining, } \\
\text { Rlchland, } \\
\text { Wash. }\end{array}$ & $\begin{array}{l}\text { No olgnificant contamination out- } \\
\text { side the laboratory room; glove } \\
\text { box breached, room contaminat ion } \\
\text { up to } 26,000 \mathrm{~d} / \mathrm{s} /(\mathrm{m} / \mathrm{n})\left(\mathrm{cm}^{2}\right) \text {. }\end{array}$ \\
\hline $4 / 15 / 59$ & Fire & $\begin{array}{l}\text { Los Alamos } \\
\text { Scientific } \\
\text { Laboratory, } \\
\text { New Mexico }\end{array}$ & $\begin{array}{l}\text { Heavy Pu contamination of the room; } \\
\text { some outside on the ground to } \\
20,000 \mathrm{~d} 1 \mathrm{~g} /(\mathrm{mln})\left(\mathrm{cm}^{2}\right) \text {. }\end{array}$ \\
\hline
\end{tabular}

11/59 Explosion Oak Ridge
Nat. Lab., Nenn.

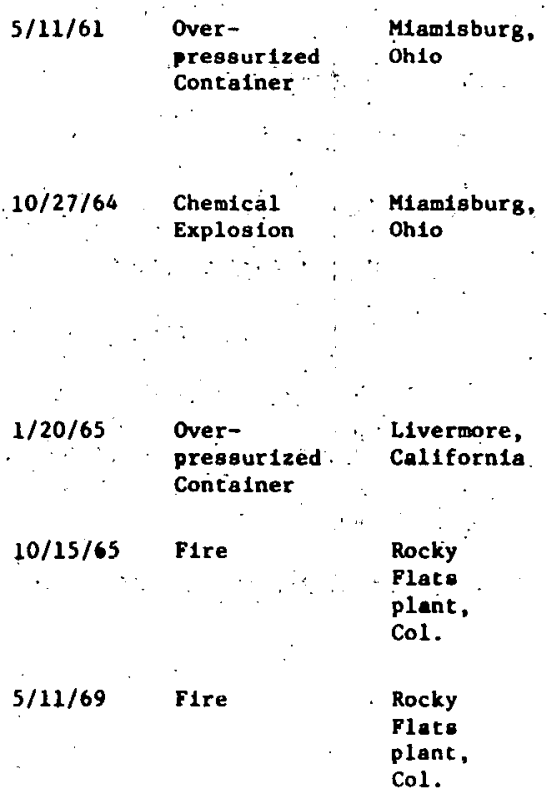

$150 \mathrm{~B} \mathrm{Pu} \mathrm{in} \mathrm{aqueous} \mathrm{solution} \mathrm{was}$ ejected from the equipment into the cell. $0.6 \mathrm{~g}$ was blown through the cell door. Outside local contamination was extensive. Cell ventilation filters removed $1.5 \mathrm{~g}$ and did not allow release via, this path to the plant stack.

Room contaminated by leak through glove box vent. No environmental contamination.

Pu escaped from the glove box, and extensive contamination of the bullding resulted from the spread. of $\sim 1 / 2 \mathrm{~g}$ of $238 \mathrm{Pu}$.

Some Pu discharged through the stack. No contaminat ion was: detected outside the bullding.

Activity outside the buldding was less than 250 counts/(min) $\left(\mathrm{cm}^{2}\right)$; contamination of the building extensive relative to $8-h r$ concentration limit.

Some contamination of the roof. but no clear evidence of external contamination from the very large fire, partly because the Pu background around the plant was high as a result of earlier Pu storage.
Too-rapid withdrawal of a sefety rod resulted in criticality and bolling surge. No ventilation syetem damage.

Spontaneou Ignition of combugt tble waste in storage. [Apparently no ventilation gyotem provided.]

$900 \mathrm{~g}$ of metal Pu residues caught fire. IVentilation gystem role not clear.]

800 g Pu metal-contalning briquetce opontaneously. Ignited and burned completely in a glove box.

$22 \mathrm{~kg}$ of $\mathrm{Pu}$ In metal form spontaneously ignited in a glove box. After $15 \mathrm{~min}$, ventllation was stopped.

Explosion, apparently from air and possibly an organic solvent from sparks from Pu metal machining.

Welding repair of metal near an asbestos filter allowed spark to Ignite Pu dust (possibly metal Pu) collected on a filter from 5-yr Pu production. Burning of combustible material on the filter resulted in destroyting the filter completely.

A chemical exploston occurred In an evaporacor inside a shielded cell.

A leak in a vacuum connection to a scrubber for the glove box operation resulted in the scrubber venting to the room. A $238^{\mathrm{Pu}}$ precipitate was being dried in the glove box.

$17 \mathrm{~g}$ of $238 \mathrm{Pu}$ was belng processed In a glove box having a methinolair.atmosphere. It, is belleved that a hot plate lanited the atmosphere and led to an explosion which pressurized the glove box and tore $81 x$ gloves from the gluve ports.

Pu-contaminoted gas was released

to a ventilaced hood and was diacharged to the atmosphere through a high atack.

011y Pu metal ch1ps and lache turnings caught fire from a spark. $30 \mathrm{~g}$ of $\mathrm{Pu}$ was widely dispersed in the bullding, apparently as very fine acrosol.

A fire swept through a product loil building, causing about $\$ 50$ million damage. The filters. apparently caught all the contaminants released during the fire. 
number of studies have been completed on experience with plutonium and the probable difficulties to be encountered: Plutonium particulates as released from fuel cycle operations have been characterized, ${ }^{8}$ based on a number of measurements made at different sites; ${ }^{9}$ the parameters which influence such effluents, particularly at fuel fabrication plants, have been assessed; ${ }^{10}$ the dangers of transporting plutonium have been estimated. ${ }^{11}$ Reference 12 contains the proceedings of a conference on safety in plutonium handling facilities. The most substantial discussion of effluents associated with the thorium- ${ }^{233} \mathrm{U}$ fuel cycle is the Final Environment Statement on the Light Water Breeder Reactor Program. ${ }^{13}$

For any possible release point, it is important to have the capability of monitoring any releases at the level of the limits imposed by existing standards, which standards are discussed immediately below. This discussion is followed by an outline of the possible objectives of monitoring programs.

\subsection{Standards}

Standards exist for the exposure to radiation of: radiation workers, individual members of the general public, and of populations ${ }^{*}$ as a whole. These standards are stated both in terms of dose equivalent (as measured in rems) to the whole body and to specific organs, and in terms of concentrations of individual radionuclides. (See glossary for a definition of terms.) These concentrations have been established both as levels in some environmental media to which humans are exposed, as well as maximum permissible amounts which may be carried internally ("body burdens"). The most generally used standards are the recommendations of the International Commission on Radiation Protection (ICRP). Of the ICRP recommendations, the most important are the maximum permissible dose equivalent for occupational exposure ( 5 rem per year), for an individual member of the general public (0.5 rem per year), and for a population ${ }^{*}\left(0.17\right.$ rem per year). ${ }^{14}$ These exposure limits are directly applicable for external radiation. However, they cannot be applied to internal radiation due to inhaled or ingested radioactive materials. For such materia1s, two different criteria are used, one for boneseeking radionuclides, for which a maximum permissible body burden (MPBB) is based on a comparison with ${ }^{226} \mathrm{Ra}$ and its daughters, and another for other radionuclides, for which the MPBB is equal to the amount which would deliver specified doses to a critical organ, i.e., the one which is most susceptible to radiation damage under the conditions of interest. The first criterion, for example, is applied to bone-seeking soluble plutonium, the second to uranium. These maximum

A "population" is some appropriately large sample of the general public. 
burdens and concentrations are typically stated for occupational exposures; according to the ICRP, the limits applicable to individual members of the general public and to populations are to be obtained by dividing by 10 and 30 , respectively (as above for the 5, 0.5, and 0.17 rem for whole body exposure).

Although the ICRP makes recommendations for dosage and concentration limits, actual responsibility for establishment and enforcement of standards resides with individual national bodies. The responsible organizations in the United States are currently the National Committee on Radiation Protection and Measurenents, a private organization, which deals with the scientific and technical aspects of radiation protection, and the U. S. Environmental Protection Agency, which is responsible for the formulation and execution of standards, except as this function is preempted by other agencies. In many areas of radiation protection and standards, particularly in nuclear power, the cognizant organization is the Nuclear Regulatory Commission (NRC). Perhaps the most significant extension by the NRC of the standards recommended by the ICRP is that, rather than permitting nuclear. power facilities considerable latitude in the amount of radioactivity released, as long as basic limits (such as the $500 \mathrm{mrem} /$ year for a member of the general public) are not exceeded, the NRC requires that emissions be "as low as is reasonably achievable". 15 In licensing, the NRC considers in detail the technology that is currently available for controlling emissions. The philosophy employed is that the controls are required to be "cost effective", based on a dollar value assigned to each man-rem of exposure. Within such a context, it is appropriate that the capability of monitoring radioactive emissions match the need for information, as a basis for both control and 1icensing.

Appendix A contains excerpts from published regulations, specifying limits on emissions from nuclear facilities and on radionuclide concentrations in air and water. Limits of three kinds are given:

1. Limits on the dose equivalent permitted individuals and populations surrounding such facilities. The NRC has more severe limits than those recommended by the ICRP. The "as low as is reasonably achievable" standard is currently interpreted to 1 imit exposure of any member of the general public to $5 \mathrm{mrem} / \mathrm{yr}$ from routine effluents. This is a factor of 100 . lower than the ICRP limit of $0.5 \mathrm{rem} / \mathrm{yr}$. It is also much less than the $0.1 \mathrm{rem} / \mathrm{yr}$ typically received from natural background radiation. linally, most of the exposure from routine releases from nuclear facilities arises from noble gas fission products. lixperience has been that the $5 \mathrm{mrem} / \mathrm{yr}$ standard can be met at the boundary of opcrating nuclear facilities. Large-scale world-wide nuclear development, with 1000 gigawatts capacity operating, would subject the average individual to a skin dose of: 
approximately $1 \mathrm{mrem} / \mathrm{yr}$ from krypton 85.16 This would dominate the dose received from routine effluents.

2. Limits (recently proposed by the EPA) on exposure of any member of the general public from planned radioactive discharges, and on total emissions of specified radionuclides from the entire uranium fuel cycle (i.e., the LWR cycle without plutonium recycle).. The specific limit on alpha-emitting transuranic discharges (including plutonium) would be 0.5 millicuries (mCi) per gigawattyear (see Appendix A). There is no specific 1imit on discharges of uranium. Most transuranic discharges would occur at the reprocessing plant. The Nuclear Fuel Services plant discharged approximately $4 \mathrm{mCi}$ of alpha emitters per gigawattyear; ${ }^{8}$ the smaller EPA 1 imit would be based on the improved technology that is currently available.

3. Limits on concentrations of specified radionuclides in environmental media. These are typically the quantities of most direct interest in release monitoring (although ultimate objectives are typically broader, as discussed below). Appendix A gives maximum permissible concentrations for a wide range of radionuclides.

A detailed introduction to the physical and chemical properties of uranium and plutonium, and to their health effects and related protection criteria, is given in Ref. 17. The uranium and plutonium isotopes of greatest importance are alpha emitters, which are typically rather long-lived, and thus can persist in the environment for long periods of time. Table 2-2 gives maximum permissible concentrations for the important alpha-decaying isotopes of uranium, plutonium, and radium. The two environmental media for which MPC's are specified are air and water, and it is important to note that the results depend strongly on whether the isotope is in soluble or insoluble form, particuarly for plutonium. For air, most of the MPC's are on the order of $40 \times 10^{-15} \mathrm{Ci} /$ liter. The most notable exception is soluble plutonium, which is $0.6 \times 10^{-15} \mathrm{Ci} / 1$ iter. (Note that these are given as 168-hour week occupational MPC's. MPC's for a member of the general public would be obtained by dividing by 10.) A11 the MPC's are stated in terms of activity. per unit volume, and the results vary with isotope because of different chemical activity, clearance rate, and total energy deposited when the nuclide (and its daughters, in the case of ${ }^{232} \mathrm{U}$ ) decay. As we will see in Section 3 , the LWR, IMFBR,

*As yet no 1 imit has been proposed for fuel cycles involving recycle of plutonium or use of thorium. 
Table 2-2. Maximum permissible concentrations (168-hour occupational)* for radium, uranium, and plutonium alpha-emitters

\begin{tabular}{lcl} 
ISOTOPE & $\begin{array}{c}\text { AIR } \\
\left(10^{-15} \text { Curies/1iter }\right)\end{array}$ & $\left(10^{-6}\right.$ WATER \\
\hline $226 \mathrm{Ra}$ & S 10 & S 0.0001 \\
& I 20 & I 0.3 \\
$232_{\mathrm{U}}$ & S 30 & S 0.4 \\
& I 9 & I 0.4
\end{tabular}

233,234

$\begin{array}{rrr}235,236_{U} & \text { S } 200 & \text { S } 0.3 \\ & \text { I } 40 & \text { I } 0.3 \\ 238_{U} & \text { S } 30 & \text { S } 0.4 \\ & \text { I } 50 & \text { I } 0.4\end{array}$

238,239

$240,242 \mathrm{Pu}$

S 0.6

$S 0.05$

I 10

I 0.3

${ }^{*} S$ is for isotope in soluble form, I for insoluble. Values are from Code of Federa1 Regulations 10 CFR 20 and NBS Handbook \#69, as quoted in Appendix A. 
and HTGR fuel cycles handle similar amounts of material by weight, and plutonium in all cases accounts for most of the activity involved. The only case where the amount of uranium activity is significantly increased as a result of reactor operations is the HTGR, which converts thoriun to ${ }^{232} \mathrm{U}$ and ${ }^{233} \mathrm{U}$; these are shortlived compared with ${ }^{235} \mathrm{U}$ and ${ }^{238} \mathrm{U}$, and therefore have higher specific activities. A summary of the physical properties of uranium and plutonium isotopes is given in Table $2-3$.

Ambient leve1s are typically much smaller than the MPC's of Table 2-2, even when the latter are reduced by a factor of 30 to the values for populations. Concentrations of uranium in air are on the order of $3 \times 10^{-18} \mathrm{Ci} / 1 \mathrm{iter}$. Concentritions in water have been found to be on the order of $3 \times 10^{-13} \mathrm{Ci} / 1 \mathrm{iter}$, although somewhat larger concentrations have been observed. In either case, the measured amounts are much less than the permissible 1imits. Most of the observed uranium arises from natural sources; uranium constitutes on the order of one millionth (by weight) of rocks, sand, etc. (See Ref. 18 for further discussion.)

Plutonium does not occur naturally. Environmental levels of plutonium are due primarily to weapons testing and space applications ( ${ }^{238} \mathrm{Pu}$ power sources). The global inventory is about $400,000 \mathrm{Ci}$, most of which has been deposited on the surface. A representative concentration of plutonium activity in surface soil in the United States is $5 \times 10^{-14} \mathrm{Ci} / \mathrm{gram}$, which is about $1 \%$ of the natura1 alpha activity from thorium and uranium. Air concentrations have been as high as $1.7 \times 10^{-18} \mathrm{Ci} /$ liter in New York (measured in 1963), still considerably less than the 1 imit of $20 \times 10^{-18} \mathrm{Ci} /$ iter of soluble plutonium for populations. Current levels are one or two orders of magnitude less than the 1963 measurements.

The various kinds of standards indicated above arise from differing problems, each with its own regulatory philosophy. Alpha-emitting uranium and plutonium have their primary importance as "internal" emitters which can do harm by being deposited in the body, whereas most whole-body exposure limits are based on "external" emitters (primarily of gamma and beta radiation). In the latter case, the regulatory position is usual1y that any exposure as measured in man-rems is harmful, the effect being proportional to the total size of the exposure and independent of the size of the population group over which the exposure is distributed. This "linear" hypothesis has been most generally applied to external emitters. There is a general convection (albeit with dissenters) that for internal emitters a "threshold", a minimum exposure to a given individua1, must be exceeded before causing damage that cannot be overcome by bodily repair 
Table 2-3, Some Properties of Uranium and Plutonium Isotopes

\begin{tabular}{|c|c|c|c|}
\hline Isotope & Half-Life & Immediate Daughter & Main Decay Products and Energies \\
\hline${ }^{232} \mathrm{U}$ & 72 years & ${ }^{228} \mathrm{Th}, 1.9 \mathrm{yr}$ & $\begin{array}{ll}68 \% & 5.32 \mathrm{MeV} \alpha \\
32 \% & 5.27 \mathrm{MeV} \alpha\end{array}$ \\
\hline $233_{U}$ & $1.6 \times 10^{5} \mathrm{yr}$ & ${ }^{229} \mathrm{Th}, 7340 \mathrm{yr}$ & $\begin{array}{ll}83 \% & 4.82 \mathrm{MeV} \alpha \\
15 \% & 4.78 \mathrm{MeV} \alpha\end{array}$ \\
\hline${ }^{234} \mathrm{U}$ & $2.47 \times 10^{5} \mathrm{yr}$ & ${ }^{230} \mathrm{Th}, 8 \times 10^{4} \mathrm{yr}$ & $\begin{array}{ll}72 \% & 4.77 \mathrm{MeV} \cdot \alpha \\
28 \% & 4.72 \mathrm{MeV} \alpha\end{array}$ \\
\hline${ }^{235} 5_{U}$ & $7.1 \times 10^{8} \mathrm{yr}$ & ${ }^{231} \mathrm{Th}, 25.5$ hours & $\begin{array}{l}57 \% 4.40 \mathrm{MeV} \alpha+204,163,110 \mathrm{keV} \gamma^{\prime} \mathrm{s} \\
18 \% 4.37 \mathrm{MeV} \alpha+\gamma^{\prime} \mathrm{s} \\
25 \% \text { other } \alpha\end{array}$ \\
\hline${ }^{236} \mathrm{U}$ & $2.4 \times 10^{7} \mathrm{yr}$ & ${ }^{232} \mathrm{Th}, 1.4 \times 10^{10} \mathrm{yr}$ & $\begin{array}{ll}74 \% & 4.49 \mathrm{MeV} \alpha \\
26 \% & 4.44 \mathrm{MeV} \alpha\end{array}$ \\
\hline${ }^{237} \mathrm{U}$ & 6.7 days & ${ }^{237} \mathrm{~Np}, 2.1 \times 10^{6} \mathrm{yr}$ & $B^{-}(0.245 \mathrm{MeV}$ maximum $)$ \\
\hline${ }^{238} \mathrm{U}$ & $4.5 \times 10^{9} \mathrm{yr}$ & ${ }^{234} \mathrm{Th}, 24.1$ days & $\begin{array}{ll}77 \% & 4.20 \mathrm{MeV} \alpha \\
23 \% & 4.15 \mathrm{MeV} \alpha\end{array}$ \\
\hline${ }^{239_{U}}$ & 23.5 minutes & $239 \mathrm{~Np}, 2.35$ days & $\beta^{-}(1.29 \mathrm{MeV}$ maximum $)$ \\
\hline${ }^{236} \mathrm{Pu}$ & $2.85 \mathrm{yr}$ & ${ }^{232} \mathrm{U}, 72 \mathrm{yr}$ & $\begin{array}{ll}69 \% & 5.77 \mathrm{MeV} \alpha \\
31 \% & 5.72 \mathrm{MeV} \alpha\end{array}$ \\
\hline${ }^{237} \mathrm{Pu}$ & $0.12 \mathrm{yr}$ & ${ }^{237} \mathrm{~Np}, 2.1 \times 10^{6} \mathrm{yr}$ & electron capture \\
\hline${ }^{238} \mathrm{Pu}$ & $86 . \mathrm{yr}$ & ${ }^{234} \mathrm{U}, 2.5 \times 10^{5} \mathrm{yr}$ & $\begin{array}{llll}72 \% & 5.50 \mathrm{MeV} & x \\
28 \% & 5.46 \mathrm{MeV} & \alpha\end{array}$ \\
\hline${ }^{239} \mathrm{Pu}$ & $2.44 \times 10^{4}$ & ${ }^{235_{\mathrm{U}, 7.1 \times 10^{8}} \mathrm{yr}}$ & 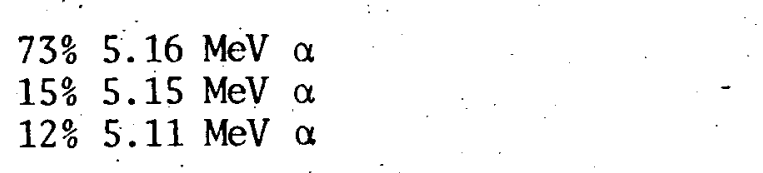 \\
\hline${ }^{240} \mathrm{Pu}$ & $6580 \mathrm{yr}$ & ${ }^{236} \mathrm{U}, 2.4 \times 10^{7} \mathrm{yr}$ & $\begin{array}{ll}76 \% & 5.17 \mathrm{MeV} \alpha \\
24 \% & 5.12 \mathrm{MeV} \alpha\end{array}$ \\
\hline $241_{\mathrm{Pu}}$ & $13.2 \mathrm{yr}$ & ${ }^{241} \mathrm{Am}, 433 \mathrm{yr}$ & $\beta^{-}(0.031 \mathrm{MeV}$ maximum $)$ \\
\hline${ }^{242} \mathrm{Pu}$ & $3.8 \times 10^{5} \mathrm{yr}$ & ${ }^{238} \mathrm{U}, 4,5 \times 10^{9} \mathrm{yr}$ & $\begin{array}{llll}76^{\circ} & 4.90 \mathrm{MeV} & \alpha \\
24 \% & 4.86 \mathrm{MeV} & \alpha\end{array}$ \\
\hline${ }^{241}{ }_{\mathrm{Am}}$ & 433 yr & ${ }^{237} \mathrm{~Np}, 2.1 \times 10^{6} \mathrm{yr}$ & $\begin{array}{ll}86 \% & 5.49 \mathrm{MeV} \alpha+60 \mathrm{keV} \gamma \\
13 \% & 5.44 \mathrm{MeV} \alpha\end{array}$ \\
\hline
\end{tabular}

From Ref. 19 
mechanisms. However, in instances involving the public, a linear point of view is often adopted - i.e., any amount of exposure may cause harm. The basic philosophy of radiation protection, as well as specific limits, undergoes continuous review by the NCRP, and it is important to realize that changes in . standards may occur at any time. In particular, possible changes in the plutonium standard are a matter of current interest.

2.4 Monitoring Objectives

In later sections, we will emphasize the physical techniques which may be used to identify plutonium and uranium in various media. However, the applicability of a particular technique is highly dependent on the requirements of the monitoring program being designed. Thus any monitoring program depends on the specified objectives and, in turn, specifies the manner in which monitoring techniques are applied. The need will ultimately amount to protection of humans or the general environment from damage due to radiation, but often resolves itself into contrasting possible emphases, which are stated here to indicate some of the possible objectives of a monitoring program:

occupational vs general public protection

source vs environmental monitoring

routine vs accidental release monitoring

continuous vs intermittent sampling and/or measurement

baseline vs incremental monitoring

activity vs exposure measurement

Occupational - General Pub1ic

As indicated above, the philosophy for protection of the radiation worker is somewhat different than for protection of the general public, and it is toward the latter goal that this discussion is primarily directed. However, it is clear that many of the same techniques are employed for occupational monitoring. In fact, it is primarily in the latter circumstances that most of our experience with monitoring techniques arises. In any case, the emphasis on the release of plutonium and uranium particulates from nuclear facilities is clearly directed toward measurement of the extent to which these substances enter the genera1 environment, thereby becoming available for possible contact with the general population.

\section{Source - Environmenta1}

Actual release of radioactivity may be monitored in two distinct fashions: by measurement either of the amount being emitted at the source, the nuclear 
facility itself, or of the amount present in the general environment into which the material is released and in which it may be dispersed. Source monitoring is a direct indicator of the actual amount released. Environmental monitoring serves a more generalized purpose of indicating how material is distributed in the environment, thereby indicating the integrated effect of past releases. It also serves as a check on source monitoring. From the point of view of protection of populations, environmental monitoring is the more fundamental, since it directly indicates the availability of radioactive nuclides for uptake by or exposure of humans. However, from a practical point of view, source monitoring is easier, simply because the activity concentrations at the release point are greater and therefore easier to measure. Moreover, the nuclear facility itself is clearly, of al1 places, the one which is most likely to give an indication of a release. A complete monitoring program will necessarily employ both points of view in a complementary fashion. Each approach will require instrumentation and techniques adapted to its own requirements.

Routine - Accidental

In principle, either source or environmental monitoring can detect both routine radioactive effluents and accidental releases. For either approach, though, requirements on response time and ultimate utilization of the resulting information will differ. Basica1ly, routine emission data may be utilized for relatively long-range planning and reporting. But depending on the extent of an accidental release, information on the amount and type of release will be employed rapidly, not only to assist in stopping the release, but - more importantly - as a possible basis for rapid action in population protection. Because of the latter rapid response requirement, monitoring for accidental releases is most easily implemented at the source of the release, where concentrations are higher, and therefore more susceptible to rapid measurement, and where facilities for relatively continuous monitoring are more likely to be available. Moreover, since it is the nuclear facility that is the source of the release, and dispersal of any release takes a period of time, which will depend on local conditions, monitoring at the source can more effectively forewarn populations. which may be subject to exposure in the absence of protective measures. However, the requirements for emergency response do not lessen the need for measurement systems intended to check compliance with routine emission standards, since these are designed for long-term environmental protection. 
Continuous - Intermittent

Specific monitoring systems are designed with sampling and measurement time schedules which vary in fundamental ways. To name some possibilities:

- continuous sampling with continuous measurement. The carrier medium (such as air or water) is sampled or collected continuously, as with an air filter, and the material being collected is monitored continuously. This may even involve an alarm capability - i.e., an alarm is raised if the amount of radioactivity collected (or even the rate of collection) exceeds specified limits.

- continuous sampling with intermittent measurement. At specified times, the sample; which has been collected on a continuous basis, is monitored for the amount of radioactivity collected.

- intermittent sampling accompanied by intermittent measurement. Examples of this are typical soil sampling, or the special samples which may be taken in the event that continuous sampling techniques indicate as abnormality.

We will make reference to these differing possibilities in our discussion of individual techniques.

Baseline - Incrementa1

From the point of view of environmental monitoring; it is important to have established a baseline with which subsequent measurements may be compared. This baseline may be established at two levels: Measurements may be taken prior to the establishment of any facilities which may affect activity concentrations at the place of interest; this measurement would then establish a "pre-nuclear" activity concentration, and thus a fundamental baseline, against which subsequent changes (presumably increases) can be compared. At a second level, one can measure the ambient amounts of activity after facilities have been established, taking care that the measurements are made when the emissions are at routine levels. This would establish an operating baseline. However, consideration must be given to the fact that radioactivity can persist in environmental media, once released. As a result, any operating baseline may change slowly, depending not only on the current routine release rate, but also on the history of releases. In any case, it is with respect to some sort of established baseline that any environmental measurements must be made. For a particular activity and a particular instrunent or technique, this baseline may effectively be zero due to instrumental limitations, so that any positive result would indicate an increase in the environmenta1 level of that activity. But for environmental measurements, where releases may make small but important changes in activity leve1s, it is important that a baseline have been established. 
Activity - Exposure

The basic function of any monitoring instrument and of a monitoring program is to measure the level of some activity. In every case, though, this objective must be considered in the light of the ultimate purpose of such programs, i.e., to indicate actual or potential exposure of living organisms. Any complete monitoring program will measure the amount and character of activity releases down to some accepted baseline level. But a given amount of radioactivity which has a form that is relatively inaccessible to organisms for some physical or chemical reason, or which has an extremely short half-life, does not constitute as great a danger as radioactivity satisfying neither of these criteria. Priorities of a release monitoring program should be set accordingly.* Ultimately, in fact, a monitoring program should include an algorithm constructed for weighting releases or environmental levels in a way to indicate ultimate exposures. This derivative information would go a step beyond the specific question of release monitoring.

*As examples, some beta decaying species of uranium in irradiated fuel have very short half-lives and thus could present only a short-term danger. On the other hand, very large plutonium particulates are in a relatively benign form, since they are not easily dispersed or deposited in humans. Thus their danger is not immediate, but must be reckoned in terms of a general increase in environmenta1 alpha emitters. 
REFERENCES for Section 2 .

1. Environmental Analysis of the Uranium Fuel Cycle (3 Volumes, USEPA Report PB-235 806, Oct. 1973.

2. The Safety of Nuclear Power Reactors (Light Water - Cooled) and Related Facilities, USAEC Report WASH-1250, July 1973.

3. A, V. Nero, A Guidebook to Nuclear Reactors, Lawrence Berkeley Laboratory Report LBL-5206, May 1976.

4. T. H. Pigford, et al., Comprehensive Standards: The Power Generation Case, USEPA Report 68-01-0561, March 1975.

5. D. C. Hunt, Restricted Release of Plutonium - Part I. Observational Data, Nuclear Safety 12, 85-89 (1971).

6. Plutonium and Other Transuranium Elements: Sources, Environmental Distribution and Biomedical Effects, USAEC Report WASH-1359, Dec. 1974.

7. Occupational Hea1th Experience with Uranium, USERDA Report ERDA-93, April 1975.

8. W. B. Seefeldt, W. J. Mechan, and M. J. Steindler, Characterization of

Particulate Plutonium Released in Fuel Cycle Operations, Argonne National Laboratory Report ANL-75-78, May 1976.

9. J. C. Elder, M. Gonzales, and H. J. Ettinger, Plutonium Size Characteristics, Health Physics 27, 45-53 (1974).

10. J. M. Selby, et a1., Considerations in the Assessment of the Consequences of Effluents from Mixed Oxide Fuel Fabrication Plants, Battelle Pacific Northwest Laboratories Report BNWL-1697 Rev. 1, June 1975.

11. The Risk of Transporting Plutonium Oxide and Liquid Plutonium Nitrate by Truck, Battelle Pacific Northwest Laboratories Report BNWL-1846, Oct. 1975.

12. Proceedings of the Rocky Flats Symposium on Safety in Plutonium Handling Facilities, USAEC Division of Technical Information Report CONF-710401, 1971.

13. Light Water Breeder Reactor Program Environmental Statement, USERDA Report ERDA-1541 June 1976.

14. ICRP-6, Pub1ished for the International Commission on Radiological Protection by Pergammon Press, New York.

15. Code of Federal Regulations 10 CFR 50, Appendix I, Government Printing Office.

16. Siting of Fuel Reprocessing Plants and Waste Management Facilities, Oak Ridge National Laboratory Report ORNL-4451, July 1971.

17. H. C. Hodge, J. N. Stannard, and J. B. Hursh (editors), 'Uranium, Plutonium, Transplutonium Elements", Springer-Verlag, New York, 1973. 
18. Survey of Instrumentation for Environmental Monitoring, Lawrence Berkeley Laboratory Report LBL-1, Vo1, 3: Radiation, Oct. 1973.

19. C, M.Lederer, J. M. Hollander, and I. Perlman, Table of Isotopes (6th edition), Wiley, New York, 1967. 
3. TICCHNIQUES FOR IDENTIFICATION OF URANIUM AND PLUTONIUM

3.1 Chemical and Isotopic Composition

The most obvious means of identifying uranium and plutonium is the radiation associated with the radioactive decay of the various isotopes. Their principal modes of decay are given in Table 2-3. We should note, in addition, that some of the techniques we shall mention may depend on the radioactive properties of daughters of these nuclides. In addition to their characteristic radiation, uranium and plutonium isotopes may, in principle, be identified by their mass, their characteristic atomic excitations, or even their chemistry (although this last is rarely useful by itself),

Chemical form does, however, have a strong influence on maximum permissible concentration, as noted in Section 2. It can also directly affect the effectiveness of monitoring techniques, particularly those involving chemical transformations. For such techniques, the most conservative assumptions are often made as to chemical form. For example, it may be assumed that the radionuclide of interest is incorporated in a relatively insoluble compound, making it difficult to bring into solution, and therefore to monitor. On the other hand, the MPC, that is applied may be a more restrictive one associated with soluble compounds, even though this contradicts the previous assumption.

In a similar way, isotopic composition affects the permissible concentrations, since these are tied directly to specific isotopes. More subtly, various isotopes interact to a1ter effectiveness of certain monitoring techniques, since the measurables (whether decay radiation, secondary radiation, or other parameters) may interfere with one another or may even augment the technique's effectiveness by providing more easily recognizable results.

Depending on the particular part of the fuel cycle being considered, isotopic mixture varies strongly. Natural uranium, prior to processing at the mill, consists almost entirely of ${ }^{238} \mathrm{U}\left(99.3^{\circ}\right)$ and ${ }^{235_{U}}\left(0.7^{\circ}\right)$, together with a smaller concentration of decay daughters (including ${ }^{234} \mathrm{U}$ ); this concentration will depend, of course, on the history of the uranium-bearing material since, for example, elements with different chemistry than uranium may have been leached at varying rates or, beginning with radon (a gas), may have diffused from the site of the uranium parents. During processing, the daughters other than ${ }^{234} \mathrm{U}$ are essentially ail removed, so that they hecome of littlc consequence in monitoring (except that, as discussed below, ambient levels of the daughters may constitute important backgrounds, interfering with measurements). Beginning with the enrichment plant, 
the percentage content of ${ }^{235} \mathrm{U}$ increases in an amount that depends on the type of reactor being supplied, At the next stage; the fuel fabrication plant, the uranium is converted to a ceramic dioxide, after which the uranium is present in a relatively insoluble form. Residence in a reactor, of course, changes the isotopic concentration markedly, the most notable effects being a decrease in the percentage of ${ }^{235} \mathrm{U}$ and an introduction of ${ }^{236} \mathrm{U}$ via neutron capture by ${ }^{235} \mathrm{U}$. At the same time, plutonium will be produced, primarily as a result of neutron capture by a few percent of the ${ }^{238} \mathrm{U}$. As the presently most important example of these transformations, we present in Table 3-1 typical percentage compositions and yearly curie amounts in these mixtures for a $1000 \mathrm{MWe}$ light-water reactor assuming an $80 \%$ capacity factor. Most of the $1.2 \times 10^{5} \mathrm{Ci}$ of alpha activity generated per 800 MWe-year is contributed by ${ }^{238} \mathrm{Pu}$. It is estimated that $1 / 2$ to $1 \%$ of these materials would be injected into the waste stream at the reprocessing plant, due to incomplete extraction.

On the other hand, in a thorium fuel cycle, the predominant uranium isotope (by mass) emerging from the reactor would be ${ }^{233} \mathrm{U}$, with the major activity being contributed by ${ }^{232} \mathrm{U}$. As an example, we give in Table 3-2 the isotopic composition of uranium reprocessed one year after removal from an ITGR at equilibrium, where it is seen that about $2000 \mathrm{Ci}$ each of ${ }^{232} \mathrm{U}$ and ${ }^{233} \mathrm{U}$ are generated. The uranium fuel supplied to the plant is recycled material, supplemented by uranium enriched to $93.5 \%{ }^{235} \mathrm{U}$. The composition of the plutonium from such a system is also given.

The total mass of this plutonium is much smaller than that generated in the LWR, since so little ${ }^{238_{U}} \mathrm{U}$ is supplied to the HTGR. Most of this plutonium is generated by neutron capture on ${ }^{235} \mathrm{U}$. However, since this plutonium would probably not be extracted at the reprocessing plant (because of its small total mass of fissile), about two orders of magnitude more plutonium alpha activity finds its way into the high-level wastes, than in the LWR cycle. Furthermore, the total amount of plutonium alpha activity from an ITGR, about $1.8 \times 10^{5} \mathrm{Ci}$, is greater than that from a uranium-fueled LWR. Again this is dominated by ${ }^{238} \mathrm{Pu}$.

The composition of plutonium discharged from reactors which actually use it as a fuel will differ considerably from that given above for discharge from a uranium-fueled LWR. The difference for plutonium recycle into an I.WR will be that the percentage of plutonium isotopes heavier than 239 (such as 240 and 241) will increase, due to an effectively greater time available for 
Table 3-1, Yearly volume and composition of fuel materials supplied to and extracted from a $1000 \mathrm{MWe} 1 \mathrm{ight}$-water reactor (uranium-fueled) operated at $80 \%$ capacity factor ${ }^{*}$ (from Ref, 1 ).

\begin{tabular}{|c|c|c|c|}
\hline Isotope & $\%$ Composition & $\operatorname{mass}(\mathrm{kg})$ & Activity (curies) \\
\hline \multicolumn{2}{|c|}{ URANIUM SUPPLIED } & & \\
\hline 234 & 0.035 & 9.6 & 59.5 \\
\hline 235 & 3.3 & 898 & 1.9 \\
\hline 238 & 96.7 & 26,300 & 8.8 \\
\hline \multicolumn{2}{|c|}{ URANIUM EXTRACTED } & & \\
\hline 234 & 0.012 & 3.1 & 19.2 \\
\hline 235 & 0.83 & 213 & .0 .46 \\
\hline 236 & 0.44 & 113 & 7.1 \\
\hline 238 & 98.7 & 25,400 & 8.45 \\
\hline \multicolumn{2}{|c|}{ PLUTONIUM EXTRACTED } & & \\
\hline 236 & 0.00010 & 0.00025 & 132 \\
\hline 238 & 2.43 & 5.9 & $1.00 \times 10^{5}$ \\
\hline 239 & 58.4 & 142 & $8.72 \times 10^{3}$ \\
\hline 240 & 24.0 & 58.6 & $1.29 \times 10^{4}$ \\
\hline $241^{\dagger}$ & 11.2 & 27.4 & $2.79 \times 10^{6}$ \\
\hline 242 & 3.9 & 9.5 & $3.72 \times 10^{1}$ \\
\hline
\end{tabular}

* The quantities extracted are given after processing, assuming a 150 day holdup before shipment to the reprocessing plant.

${ }^{\dagger} 241$ Pu beta decays, and is not considered in the MPC's of Table 2-2. 
Table 3-2, Yearly volume and composition of fuel materials extracted from a 1000 MWe HTGR at equilibrium, operated at $80 \%$ capacity factor ${ }^{*}$ (from Ref. 1 ),

$\Xi$

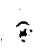

Isotope

URANIUM EXTRACTED

232

233

234

235

236

238

236

238

239

240

$241^{\dagger}$

242 mass $(\mathrm{kg})$

0.11

186.7

78.4

50.3

112.9

34.6

$3.2 \times 10^{-5}$

10.5

64.9

14.4

8.5

2.33

1.38

6.0

0.97

6.2
Activity (curies)

2296

1769

485

0.1

7.2

0.1

17

$1.77 \times 10^{5}$

$1.43 \times 10^{2}$

$3.03 \times 10^{2}$

$9.89 \times 10^{4}$

4

* fueled with thorim + recycle uranium at equilibriun; quantities present 1 year after discharge.

tBeta decays 
multiple neutron capture. But in either case, the plutonium isotopes of greatest biological significance soon after discharge will be ${ }^{238} \mathrm{Pu}$, formed by multiple neutron capture on ${ }^{235} \mathrm{U}$, and ${ }^{241} \mathrm{Pu}$, from capture on ${ }^{238} \mathrm{U}$. The first of these has an 86 year half-1ife and alpha decays, the second has a 13.2 year half-life and beta decays to ${ }^{241} \mathrm{Am}$. The alpha activity of plutonium from these LWR's is still dominated by ${ }^{238} \mathrm{Pu}$ because of its high specific activity. The other major projected use of plutonium is in the fuel cycle of the fast-breeder reactor. Because fuel supplied to the breeder will have very little ${ }^{235} \mathrm{U}$, less of the lighter plutonium isotopes is produced in the breeder than in an LWR. In particular, the percentage of ${ }^{238} \mathrm{Pu}$ decreases considerably. For purposes of comparison, in Table 3-3 we give the percentage composition and yearly curie amounts of plutonium discharged under three possible schemes: A uranium-fueled LWR, a "mixed-oxide" (uranium and plutonium)-fueled LWR, and an LMFBR. Note that the plutonium's specific alpha activity is less for the fast-breeder case than for the others, primarily because it produces 1 ittle ${ }^{238} \mathrm{Pu}$.

It is important to note, though, that these compositions only apply to materials in the fuel stream. The high-level wastes discharged from the reprocessing plant will initially have the same isotopic composition as the fuel stream, but as the isotopes decay at different rates, this composition will change. As time passes, the relative importance of the 239,240 and 242 isotopes wil1 increase.

\subsection{Radioactive Decay Monitoring}

In monitoring uranium and plutonium via their radioactive decay, a11 three of the basic radiations, alpha, beta, and gamma, can be employed. In some cases these are directly associated with the species of interest, and in others they result from decaying daughters. In most of the uranium and plutonium isotopes of interest, the nucleus decays by emission of an alpha particle with energy between 4 and $6 \mathrm{MeV}$ (see Table 3-4, a reproduction of Table 2-3) and is followed immediately by emission of gamma and/or $x$-radiation. ${ }^{2}$ The most important exception to this is ${ }^{241} \mathrm{Pu}$, which beta decays with a 13 year half-1ife to ${ }^{241} \mathrm{Am}$.

In many cases, the primary alpha or beta radiation is not measured, particularly in situations where their relatively short range in absorbing material makes it difficult to observe them. In these cases, the gamma radiation accompanying the primary decay or sometimes the decay of the daughters may be used as the observable, primarily because the gamma radiation is usually more penetrating than the alpha or beta particles. As discussed below, one also has a choice of either detecting the presence of a particular type of radiation, or actually 
Table 3-3, Isotopic composition and activities in plutonium from yearly discharge from 1000 MWe power plants (from Ref. 1).

5

Isotope 1ight-water reactor

PERCENTAGE COMPOSITION BY MASS

$\begin{array}{cc}236 & 1.0 \times 10^{-4} \\ 238 & 2.4 \\ 239 & 58.4 \\ 240 & 24.0 \\ 241 & 11.3 \\ 242 & 3.9 \\ & \text { ACTIVITY (CURIES) } \\ 236 & 1.34 \times 10^{2} \\ 238 & 1.01 \times 10^{5} \\ 239 & 8.82 \times 10^{3} \\ 240 & 1.30 \times 10^{4} \\ 241^{+} & 2.81 \times 10^{6} \\ 242 & 37.6\end{array}$

Tota1 mass $\quad(\mathrm{kg}) \quad 246$

Total alpha activity (curies) $1.23 \times 10^{5}$

Specific alpha activity $(\mathrm{Ci} / \mathrm{gm})$
0.50
Uranium-plutonium fueled light-water reactor

Fast breeder reactor

$3.7 \times 10^{-5}$

$1.5 \times 10^{-6}$

4.3

$6.9 \times 10^{-2}$

37.2

71.7

27.8

25.1

18.6

2.4

12.1

0.76

$1.76 \times 10^{2}$

$1.59 \times 10^{1}$

$6.50 \times 10^{5}$

$2.25 \times 10^{4}$

$2.03 \times 10^{4}$

$8.51 \times 10^{4}$

$5.44 \times 10^{4}$

$1.07 \times 10^{5}$

$1.69 \times 10^{7}$

$4.67 \times 10^{6}$

$4.20 \times 10^{2}$

$5.74 \times 10^{1}$

992

2058

$7.25 \times 10^{5}$

$2.15 \times 10^{5}$

0.73

0.10

${ }^{\dagger}$ beta decays 
Table 3-4. Some Properties of Uraniun and Plutonium Isotopes *

\begin{tabular}{|c|c|c|c|}
\hline Isotope & Half-Life & Immediate Laughter & Main Docay Products and Fnorgies \\
\hline $232_{U}$ & 72 years & ${ }^{228} \mathrm{Th}, 1.9 \mathrm{yr}$ & $\begin{array}{ll}68 \% & 5.32 \mathrm{MeV} \alpha \\
32 \% & 5.27 \mathrm{MeV} \alpha\end{array}$ \\
\hline $233_{U}$ & $1.6 \times 10^{5} \mathrm{yr}$ & ${ }^{229} \mathrm{Th}, 7340 \mathrm{yr}$ & $\begin{array}{ll}83 \% & 4.82 \mathrm{MeV} \alpha \\
15 \% & 4.78 \mathrm{MeV} \alpha\end{array}$ \\
\hline${ }^{234} U$ & $2.47 \times 10^{5} \mathrm{yr}$ & ${ }^{230} \mathrm{Th}, 8 \times 10^{4} \mathrm{yr}$ & $\begin{array}{ll}72 \% & 4.77 \mathrm{MeV} \cdot \alpha \\
28 \% & 4.72 \mathrm{MeV} \alpha\end{array}$ \\
\hline $235_{U}$ & $7.1 \times 10^{8} \mathrm{yr}$ & ${ }^{231}$ Th, 25.5 hours & $\begin{array}{l}57 \% 4.40 \mathrm{MeV} \alpha+204,163,110 \mathrm{keV} \gamma^{\prime} \mathrm{s} \\
18 \% 4.37 \mathrm{MeV} \alpha+\gamma^{\prime} \mathrm{s} \\
25 \% \text { other } \alpha\end{array}$ \\
\hline${ }^{236} \mathrm{U}$ & $2.4 \times 10^{7} \mathrm{yr}$ & ${ }^{232} \mathrm{Th}, 1.4 \times 10^{10} \mathrm{yr}$ & 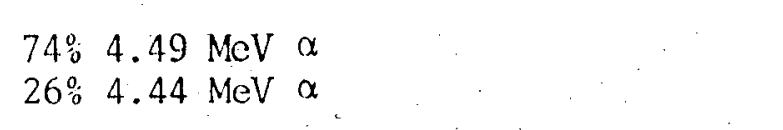 \\
\hline${ }^{237} \mathrm{U}$ & 6.7 days & ${ }^{237} \mathrm{~Np}, 2.1 \times 10^{6} \mathrm{yr}$ & $\beta^{-}(0.245 \mathrm{MoV}$ maximum $)$ \\
\hline $238_{U}$ & $4.5 \times 10^{9} \mathrm{yr}$ & ${ }^{234}$ Th, 24.1 days & 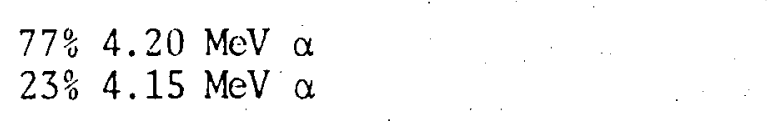 \\
\hline $239_{U}$ & 23.5 minutes & ${ }^{239} \mathrm{~Np}, 2.35$ days & $\beta^{-}(1.29 \mathrm{MeV}$ maximum $)$ \\
\hline${ }^{236} \mathrm{Pu}$ & $2.85 \mathrm{yr}$ & ${ }^{232} \mathrm{U}, 72 \mathrm{yr}$ & $\begin{array}{ll}69 \% & 5.77 \mathrm{MeV} \alpha \\
31 \% & 5.72 \mathrm{MeV} \alpha\end{array}$ \\
\hline${ }^{237} \mathrm{Pu}$ & $0.12 \mathrm{yr}$ & ${ }^{237} \mathrm{~Np}, 2.1 \times 10^{6} \mathrm{yr}$ & electron capture \\
\hline${ }^{238} \mathrm{Pu}$ & $86 \mathrm{yr}$ & ${ }^{234} \mathrm{U}, 2.5 \times 10^{5} \mathrm{yr}$ & $\begin{array}{ll}72 \% & 5.50 \mathrm{MeV} \alpha \\
28 \% & 5.46 \mathrm{MeV} \alpha\end{array}$ \\
\hline${ }^{239} \mathrm{Pu}$ & $2.44 \times 10^{4}$ & ${ }^{235} \mathrm{U}, 7.1 \times 10^{8} \mathrm{yr}$ & $\begin{array}{lll}73 \% & 5.16 \mathrm{McV} & \alpha \\
15 \% & 5.15 \mathrm{MeV} & \alpha \\
12 \% & 5.11 \mathrm{MeV} & \alpha\end{array}$ \\
\hline $240 \mathrm{Pu}$ & $6580 \mathrm{yr}$ & ${ }^{236} \mathrm{U}, 2.4 \times 10^{7} \mathrm{yr}$ & $\begin{array}{ll}76 \% & 5.17 \mathrm{MeV} \alpha \\
24 \% & 5.12 \mathrm{MeV} \alpha\end{array}$ \\
\hline${ }^{241} \mathrm{Pu}$ & $13.2 \mathrm{yr}$ & ${ }^{241} \mathrm{An}, 433 \mathrm{yr}$ & $\beta^{-}(0.031 \mathrm{MeV}$ maximum $)$ \\
\hline${ }^{242} \mathrm{Pu}$ & $3.8 \times 10^{5} \mathrm{yr}$ & ${ }^{238} \mathrm{U}, 4.5 \times 10^{9} \mathrm{yr}$ & $\begin{array}{lll}76 \% & 4.90 \mathrm{MeV} & \alpha \\
24 \% & 4.86 \mathrm{MeV} & \alpha\end{array}$ \\
\hline${ }^{241} \mathrm{Am}$ & $433 \mathrm{yr}$ & ${ }^{237} \mathrm{~Np}, 2.1 \times 10^{6} \mathrm{yr}$ & $\begin{array}{l}86 \% 5.49 \mathrm{MeV} \alpha+60 \mathrm{keV} \gamma \\
13 \% 5.44 \mathrm{MeV} \alpha\end{array}$ \\
\hline
\end{tabular}

From Ref. 2 
measuring its energy, thereby discriminating against similar radiation from sources other than the isotopes of interest: Reference 3 is a detailed survey of instrumentation for the monitoring of all types of radiation and radionuclides. A recent treatment of environmental (as distinguished from source) monitoring is given in Ref. 4 .

Several basic types of radiation detectors are available, most broadly classified as gas ionization counters, scintillation counters, and semiconductor detectors. In scintillation counters, the basic mechanism for detection of radiation is the production of visible light, which is then detected by a photomultiplier. In the other detector types, ion pairs are created by the passage of radiation, and the charge or voltage pulse resulting is counted or measured. In gas-type detectors, the induced charges are ordinary electron-atomic ion pairs from the gas itself; in the semiconductor detector, electron/hole pairs are induced in semiconducting materia1. Because the energy to create an electron-hole pair (about $3 \mathrm{eV}$ ) is so much less than the energy absorbed in the creation of a gas ion pair (order of $30 \mathrm{eV}$ ) or the energy needed in a scintillating material (hundreds of $\mathrm{eV}$ ), the intrinsic resolution of the semiconductor detector is superior to that of the other types. This is true for any given radiation since the total number of ion pairs or photoelectrons is greater and thus the fractional uncertainty due to counting statistics is smaller. However, due to other considerations, such as detection efficiency, cost, the need for a cryostat, physical ruggedness, one or the other detector type may be chosen for a given application. In certain situations, for example, resolution is of no concern, because the detector is used simply as a counter rather than as a spectrometer.

In general, the basic considerations in design of a detector for a specific purpose are the following: the efficiency with which the particular radiation is detected, the background presented by radiation from sources other than the nuclide of interest, and usually the resolution with which the radiation of interest is detected, distinguishing it both from similar radiation from other sources and from noise in the detector system. All of these considerations are influenced by any possible absorption or attenuation of the radiation by material between the source of radiation and the point at which the fundamental detection process occurs. Such absorption or attenuation will depend.strongly on the design of the particular detector and on the medium containing the radionuclide of interest.

The design of the detector(s) and associated equipment in a monitoring system depends on the specific technique chosen for identification of the interesting nuclide. The simplest type of detector system simply accumulates a total number of events during some time period. For instance, it may record the number of: 
particles or photons leaving some minimum amount of energy. There are several distinct ways in which the system may be more sophisticated: 1) Information on the energy of each event may utilized or stored, making the detector system a spectrometer. 2) Information on the time of each event may be obtained, leading to the possibility of half-1ife analysis or discrimination. 3) Discrimination may be achieved by requiring the coincidence of two or more events; since "coincidence" is defined in terms of a time period, this technique also requires measurement of event time. In this case, however, the time is measured relative to another event rather than relative to the beginning of the counting period or sample collection. Each of these techniques is commonly used in monitoring for uranium and plutonium.

The most direct radiometric technique for alpha emitters is simple alpha counting, in which the total number of counts triggering an alpha detector is accumulated. Such a simple technique, though, is subject to large uncertainties. In many cases, concentrations of other alpha emitters may be large enough to mask the counts of interest; in other cases, particularly with bulk samples, the range of alpha particles (about $6 \mathrm{mg} / \mathrm{cm}^{2}$ for $5 \mathrm{MeV}$ alphas) is so sma11 that a more difficult technique must be employed.

3.2.1 Air Monitoring

For our purposes, air monitoring is the most important class of measurements, since air-suspended material is most easily dispersed and most directly available for ingestion. The latter consideration is reflected in the relatively low air MPC's associated with isotopes of uranium and plutonium.

A universal feature of air monitoring for uranium and plutonium is the collection on an fir filter of airborne particles, the form in which these isotopes would be present. Any volume of air that could be directly monitored would be so small as to render the counting system very insensitive. Filtering concentrates the particulate content of a large volume of air into a small region, which may then be monitored directly. However, even with a typical collection system that filters about 1 liter of air per second, substantial collection and counting times must be used to measure levels approaching a general public MPC. For example, a one-hour collection at $1 \mathrm{iiter} / \mathrm{sec}$ of $1 \mathrm{MPC}$ of soluble plutonium (for which the MPC is $6 \times 10^{-17}$ curie/liter) would result in a sample yielding 0.5 disintegrations per minute, a rather small rate. The actual counting rate would be further reduced because total detection efficiency is less than 1. The resulting sensitivity for measurement of plutonium would depend on the detection 
system's ability to discriminate against background, In principle, of course, one need not separate the collection period from the counting period; on many systems, counting occurs while the activity is being collected. Because the activity is accumulated during counting ("on-1ine"), sensitivity to a given air concentration is not as well defined as for instruments where collection and counting are sepparate ("off-1ine").

MPC's for plutonium, and even for uranium, are several orders of magnitude lower than ambient air concentrations of other alpha emitters, most importantly radon-222 and its daughters. Since radon, a gas, and to a lesser extent, radon 220 , occur naturally at concentrations of 0.1 to $1 \times 10^{-12} \mathrm{Ci} / 1$ iter, and its daughters - typically found attached to particles - are efficiently collected by filters, the background against which uranium and plutonium must be measured is substantial. Figure 3-1 gives decay chains for ${ }^{222} \mathrm{Rn}$ and ${ }^{220} \mathrm{Rn}$ (daughters of ${ }^{238} \mathrm{U}$ and ${ }^{232} \mathrm{Th}$, respectively), along with energies of the principal radiations.

In spite of such backgrounds, the most common method for monitoring of plutonium and uranium in air is still filter collection followed by direct measurement. In situations where simple alpha counting is practical, the detector is typically a scintilator-photomultiplier system, where only the total number of counts is accumulated. More sophisticated systems are avaiable for discriminating against background, most of which fit into the categories mentioned above: 1) spectroscopy, 2) . Iifetime analysis, 3) associated $\beta$ measurements.

These techniques are equally applicable to monitoring of either plutonium or uranium alpha emitters. The first depends on measurement of alpha energies from isotopes of interest, on the premise that these energies differ from those due to background. The second depends directly on the short lifetime of daughters of radon compared with the relatively long lifetime of the interesting $\mathrm{Pu}$ and $\mathrm{U}$ isotopes. The third makes use of the $\beta$ particle that almost immediately precedes alpha decay of ${ }^{214}$ Po (Radium $C^{\prime}$ ). These techniques are discussed below. Additional methods depending on size segregation, radiochemical segregation, etc., will be discussed in following sections.

\subsubsection{Alpha Spectroscopy}

Figure 3-2 shows the alpha energies, and comparative strength, for certain isotopic mixtures of uranium and plutonium and for radon and daughters $\left({ }^{218} \mathrm{Po}\right.$ and $\left.{ }^{214} \mathrm{Po}\right)$ in equilibrium. We also show the normally less important radon 220 chain.

It is possible to design instruments with sufficiently good resolution to distinguish all important $\mathrm{U}$ and Pu isotopes from members of the radon chains, 


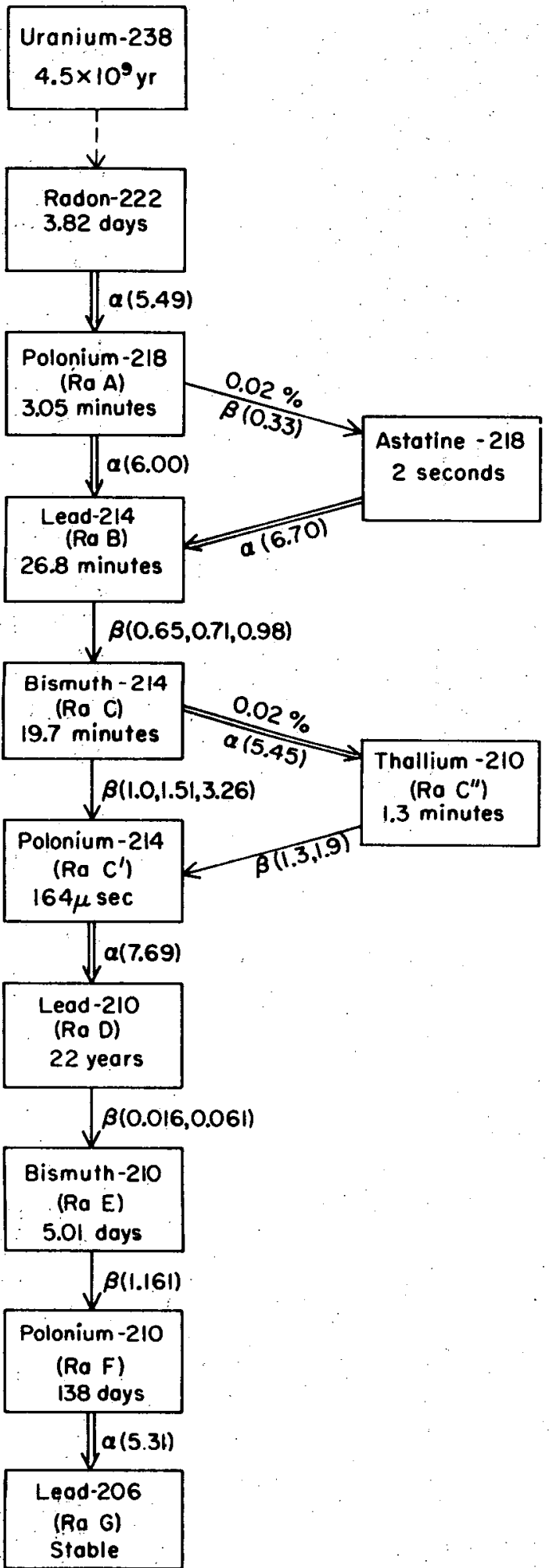

Decay Chain, Radon-222 to Lead-206 $\alpha$ and $\beta$ energies in $\mathrm{MeV}$

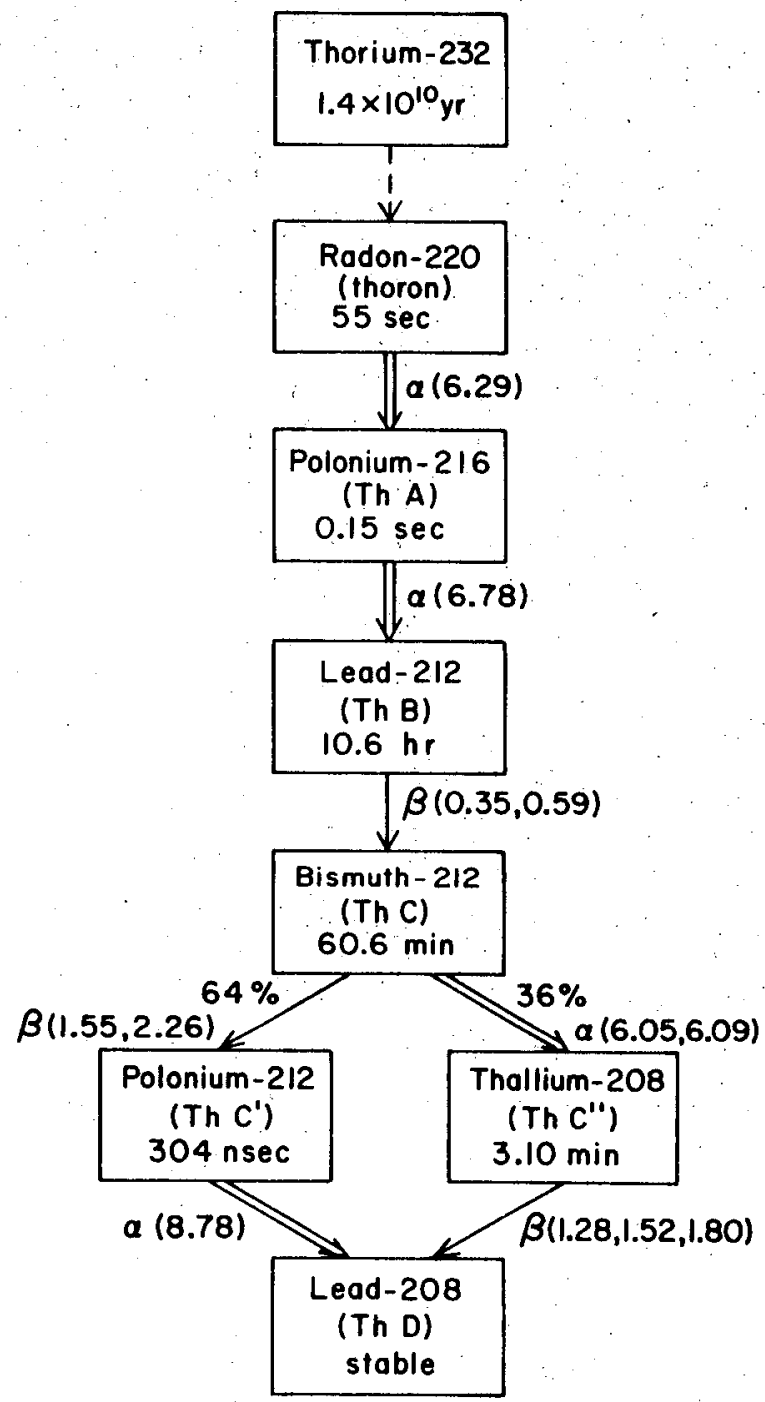

Decay Chain, Radon-220 to Lead-208 $\alpha$ and $\beta$ energies in $\mathrm{MeV}$

Fig. 3-1. Radon daughter decay chains (from Ref. 2). 

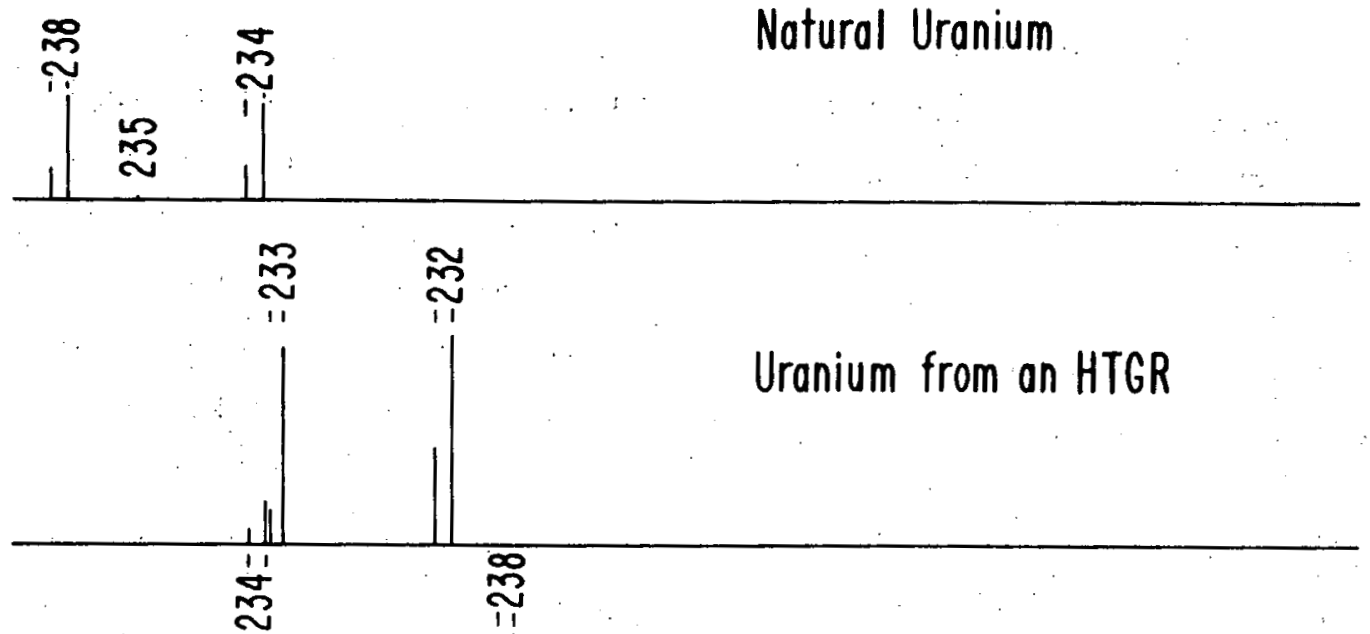

Plutonium from a Uranium-fueled LWR
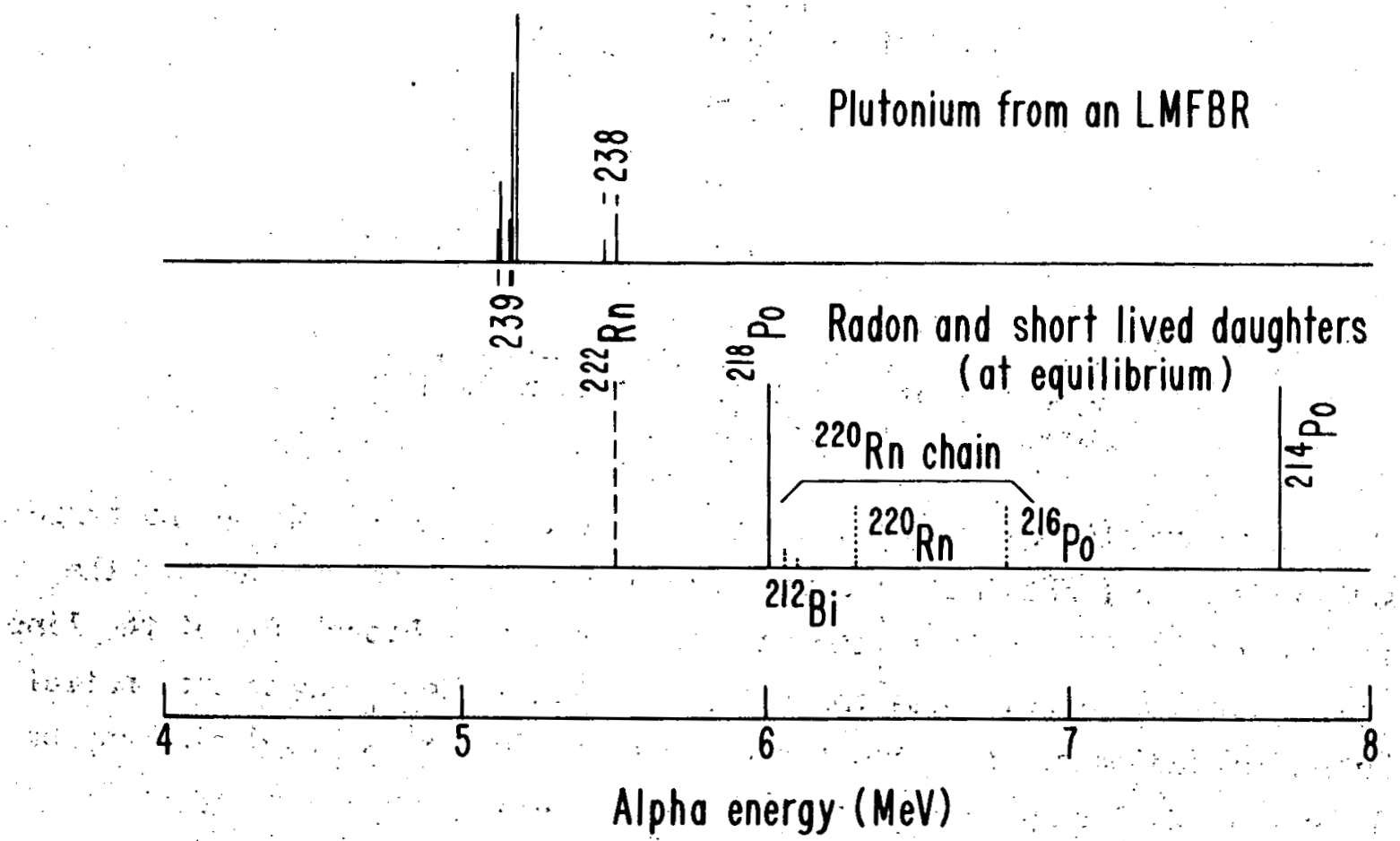

XBL $768-3263$

Fig. 3-2. Relative strength of alpha groups from typical mixtures of urinium and plutonium. For any given mixture, the height of each line is proportional to the strength of that group. The mass numbers indicate which isotopes of the given element account for each group. 
assuming that the radon gas itself is not efficiently collected from the air. However, because the alphas from radon daughters have higher energy than those from the isotopes of interest, the radon daughters will always present some background. This arises from energy degrading processes that will persist at some level. The effect of the background, whether due to radon daughters, other radiation, or noise, will decrease as resolution is improved.

Either a semiconductor detector or a gas proportional counter is generally used for alpha spectrometry. The former has better intrinsic resolution characteristics but since this optimum resolution is often unavailable in any case, the proportional counter's large size and less sophisticated physical structure may prompt its use. The optimal geometric arrangement for a semiconductor detector system places the detector itself, typically a thin circular wafer, directly in front of and paralle1 to the filter material (see Fig. 3-3).

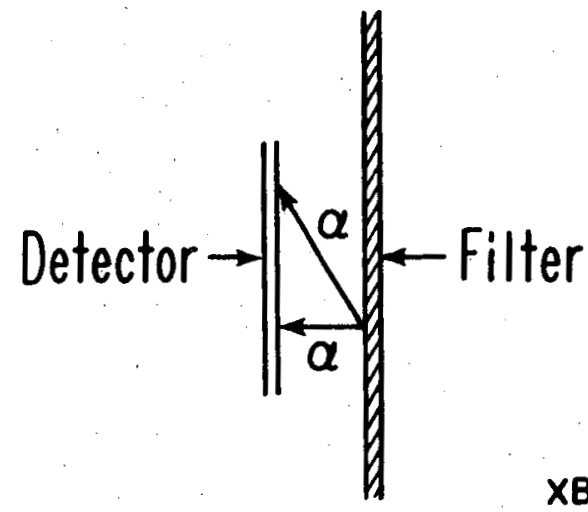

XBL $768-3257$

Fig. 3-3. Schematic arrangements for filter and semiconductor detector in an alpha counting system; possible variation in alpha path length is indicated.

Although such a detector can be used to achieve resolutions (FWH) in the range of $20 \mathrm{keV}$, this is not obtained in direct alpha counting from filters for the following reasons: 1) The filter material itself causes a broadening of the line due to energy loss as alphas pass through the material -depending on the spatial distribution of radionuclides through the filter medium, this broadening may be hundreds of keV. 2) A similar difficulty is caused by the gas which typically exists between the filter and the detector. One cm of air at atmospheric pressure has a thickness of about $1 \mathrm{mg} / \mathrm{cm}^{2}$, which can absorb about $0.75 \mathrm{MeV}$ from a $5 \mathrm{MeV}$ alpha. Since one cannot reduce the length much below $1 \mathrm{~cm}$, the straggle in this energy loss, due either to the basic energy loss process or to variations 
in the path length, can markedly degrade the system resolution. Other aspects of the manner in which semiconductor detectors are used can degrade resolution; but in the present case, the two factors above are dominant. In a continuous monitoring system any optimization of the geometry would have to take account of the need for the air-filtering action to continue during counting.

Although it is clear from Fig. 3-2 that, under most circumstances, a detector system with tens of $\mathrm{keV}$ resolution will efficiently discriminate against radon-daughters, the same is not true of a system with peak widths of hundreds of keV. This is demonstrated in results shown in Fig. 3-4 from one of the earliest solid state counter systems. ${ }^{5}$ There, even the $5.1 \mathrm{MeV}$ alpha groups from ${ }^{239} \mathrm{Pu}$ (or ${ }^{240} \mathrm{Pu}$ ) overlap with the radon $\mathrm{A}\left({ }^{218} \mathrm{Po}\right)$ group. The peaks are partially resolved but - because of the overlap - analysis has to take account of the ${ }^{218}$ po counts underlying those due to plutonium. Discrimination is quite significant but the analysis is not trivial.

It is important to note further than ${ }^{239} \mathrm{Pu}$ activity only dominates weapons grade plutonium, which has an isotopic mixture by mass of roughly $0.04 \%{ }^{238} \mathrm{Pu}$, 93.3\% ${ }^{239} \mathrm{Pu}, 6.0 \%{ }^{240} \mathrm{Pu}, 0.6 \%{ }^{241} \mathrm{Pu}$, and $0.04 \%{ }^{242} \mathrm{Pu} .{ }^{6}$ Commercial reactor plutonium mixtures were described in Section 3.1. Of these, the mixture that is most similar to weapons-grade plutonium comes from the LMFBR, where ${ }^{239} \mathrm{Pu}$ and ${ }^{240} \mathrm{Pu}$ activities of $5.1 \mathrm{MeV}$ or so dominate the spectrum, as seen in Fig. 3-2. However, in LWR plutonium, ${ }^{238} \mathrm{Pu}$ activity is most important and most easily. masked by radon daughters, an unfortunate coincidence. To some extent, a similar difficulty arises for uranium from an HTGR, where the ${ }^{232} \mathrm{U}$ activity is very important, particulärly since its MPC's are lower than the other uranium isotopes. The alphia groups from natural uranium and LWR uranium are lower in energy, so that radon and daughters do not constitute as serious a background in these cases.

Monitoring systems from commercial manufacturers are available both as continuous monitors and as "off-line" monitors. In the latter case a filter from a separate air-sampling unit is inserted into the instrument. In either case, the geometry is essentially as described above; the filter paper is most often $47 \mathrm{~mm}$ in diameter, and the counters are usually large-area (up to $750 \mathrm{mn}^{2}$ ) diffused junction or surface barrier detectors. The energy spectrum from such a system is usually similar to that of Fig. 3-4, i.e., with a resolution of many hundreds of $\mathrm{keV}$. However, the typical commercial systems, which are sma11, inexpensive, self-contained units, do not store this energy spectrum. Instead, 


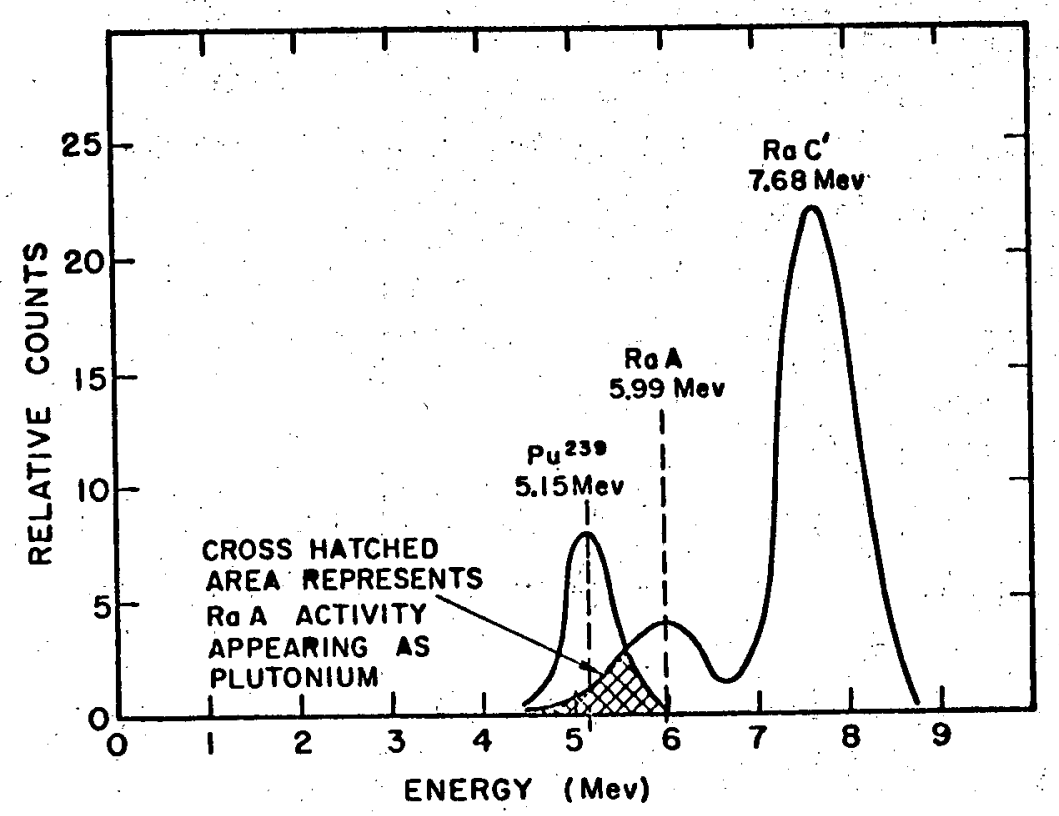

Fig. 3-4. Radium A (Polonium-218) interference in plutonium detection (from Ref. 5).

\section{Filter}

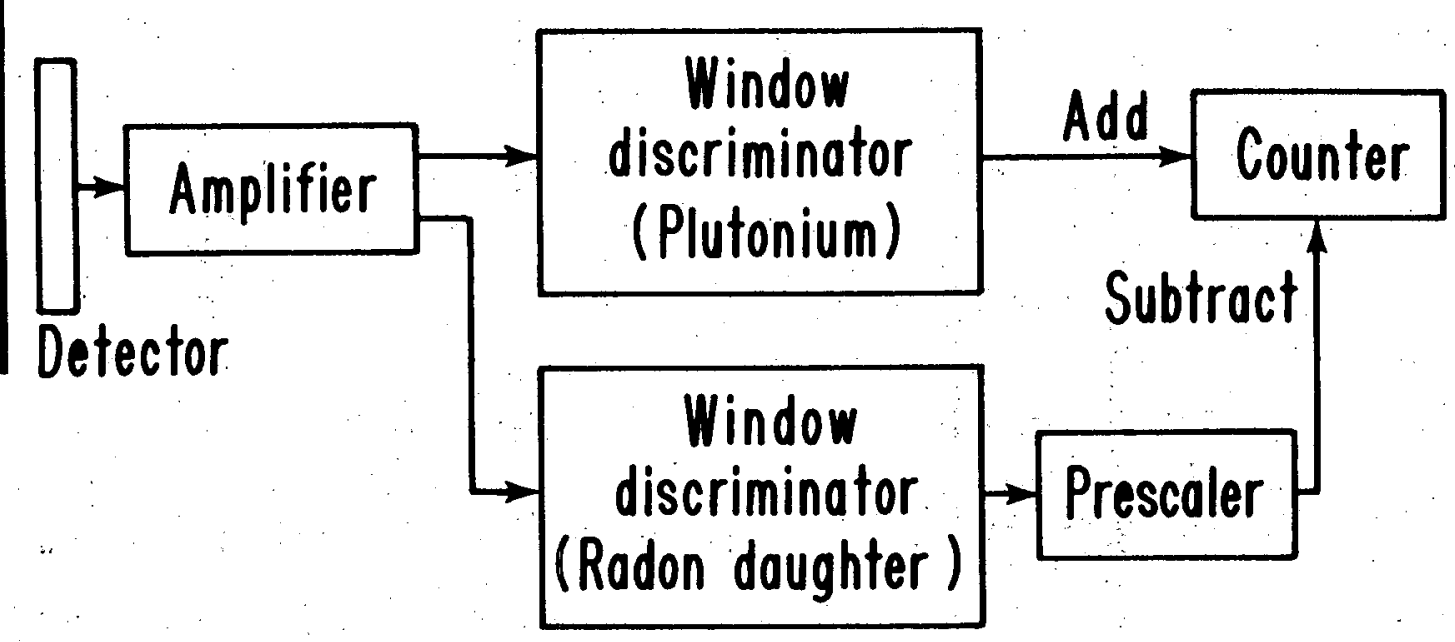

XBL 768-3259

Fig. 3-5. Schematic diagram of a simple alpha spectroscopic system with background compensation. 
the unit counts the decays within some preset energy "window" corresponding to the alphas of interest. Many units make some compensation for the presence of a portion of the ${ }^{218}$ Po decays in this window. This compensation may, for example, be accomplished by making a subtraction based on another energy window as indicated in the block diagram of Fig. 3-5. Such a device is often called a "selective alpha monitor" and may claim sensitivities as low as 1 MPC-hour (40-hour occupational) for ${ }^{239} \mathrm{Pu}$. Such claimed sensitivites are with a low confidence level, even under optimal operating conditions. Thus it would be imprudent to rely on such devices' response to such small amounts of radioactivity. They are nevertheless useful for routine occupational monitoring situations and for release measurement. When used for the latter application, these instruments typically put only a loose upper bound on quantities released. Their useful sensitivity is more in the range of $10 \mathrm{MPC}$ - hours (occupational).

Gas proportional counters could be used in the above systems in place of semiconductor detectors because, as the latter are actually used, their resolution is relatively poor. The resolution of hundreds of $\mathrm{keV}$ encountered above is not due to the intrinsic resolution of the detector, and substitution of a gas counter would yield the same result. Moreover, the latter can easily be designed to cover large filter areas. A disadvantage is that these are most suitable for "off-line" counting, since the need for a thin window can make a gas counter difficult to use in a continuous mode.

It is possible to construct an alpha-spectroscopic system from conmerciallyavailable detectors and analysis systems that has resolution and intrinsic sensitivity much superior to that described above. Attendant difficulties are the need to put the filter-semiconductor detector combination in an evacuated chamber and to devise a more sophisticated data analysis approach than the simple window-subtraction technique mentioned above. The system would also be considerably more expensive: However, this is the approach taken in many laboratory situations. See also below under radiochemical analysis.

\subsubsection{Lifet ime Analys is}

The radon daughters that constitute the most serious alpha background all have much shorter half lives (see Fig. 3-1) than the alpha-emitting $U$ and Pu isotopes. This fact may be used in a variety of ways to reduce the effect of this background. These techniques usually depend on an off-line procedure i.e., separation of the collection and counting periods. The main radon-daughter chain of interest is: 

${ }^{218}$ Po
${ }^{214} \mathrm{~Pb}$
$214 \mathrm{Bi}$
$\underset{20 \text { Minutes }}{\stackrel{B}{\longrightarrow}}$
${ }^{214} \mathrm{Po}$
$\alpha$
(Ra A) 3 Minutes
(Ra B) 27 Minutes
(Ra C) 20. Minutes
$\left(\operatorname{Ra} C^{\prime}\right) 164 \mu \mathrm{sec}$

A waiting period before counting reduces the amount of daughters present by the time counting occurs. This is particularly important for ${ }^{218} \mathrm{Po}$, which has a short half-1ife and is the isotope that is most troublesome in the alpha-spectroscopic techniques (see Figs. 3-2 and 3-4). As a result, a long collection period or a holdup period between collection and counting reduces the relative size of radon daughter background. However, to achieve good sensitivity, the holdup period may have to be very long, a condition that is inconsistent with many monitoring applications.

Alternatively, one may do a partial or complete lifetime analysis-i.e., count in successive time periods to determine the half-life composition of the collected sample. If the counting system does have a spectroscopic capability, this time dependence need only be determined for counts in the preset window of interest. Since only ${ }^{218} \mathrm{Po}$, with a 3 minute half life, would interfere, the analysis could be relatively simple. A more complete analysis would treat the full energy range (or at least that over a broad range, perhaps everything above some discriminator leve1). This fuller analysis would of course be necessary in the absence of energy information and may require a fit using several half-lives (3, 27, 20 minutes). In either case, any component of the activity that does not show a decrease with time would be attributed to possible $\mathrm{Pu}$ or $\mathrm{U}$ isotopes.

In a system in which measurement of time assumes importance, a different detector type may be chosen than in a spectroscopic system. Under these circumstances even scintillator-based systems, with their poor energy resolution, may be appropriate, particularly if energy information is no longer sought. Further, the associated electronic logic and data-analysis system will differ from that for pure spectroscopic measurements, primarily in that they will be timing, rather than energy-analysis systems. However, enphasis on timing puts no strong constraints on the detectors themselves, since the timing consists primarily of measurement of counting periods.

Lifetime analysis monitors are not generally available from commercial manufacturers. The detectors and related equipment are available, but the user must design and assemble this equipment in his own logic timing system, such as that indicated in Fig. 3-6. 


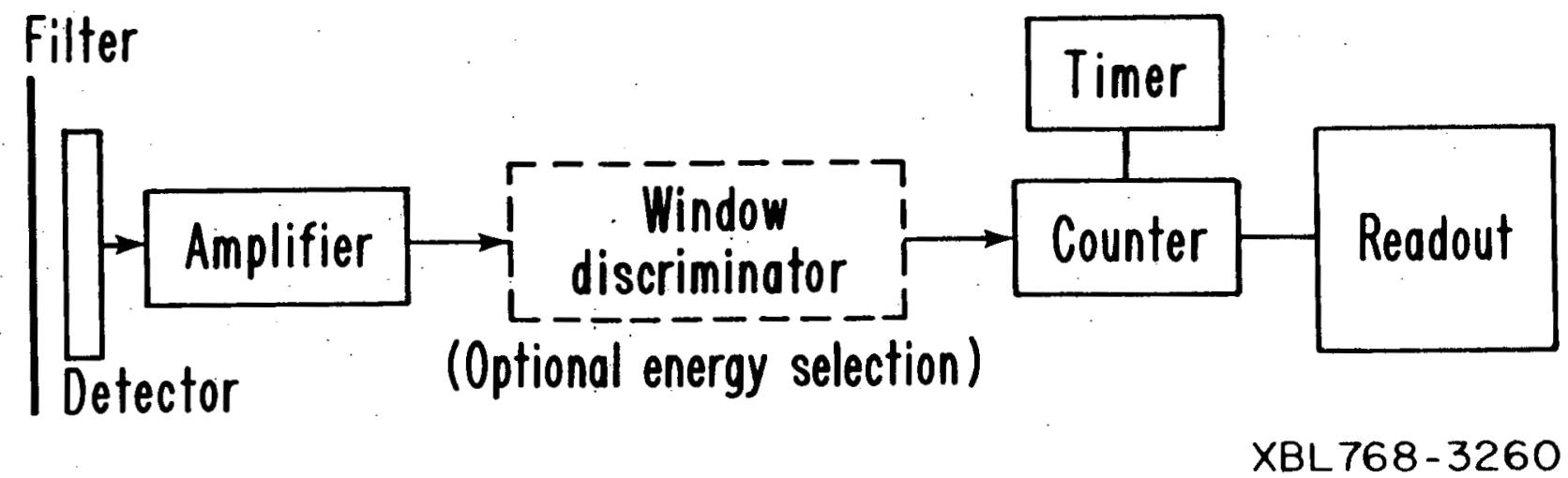

Fig. 3-6. Schematic diagram of alpha counting (or optional spectroscopy) with lifetime analysis.

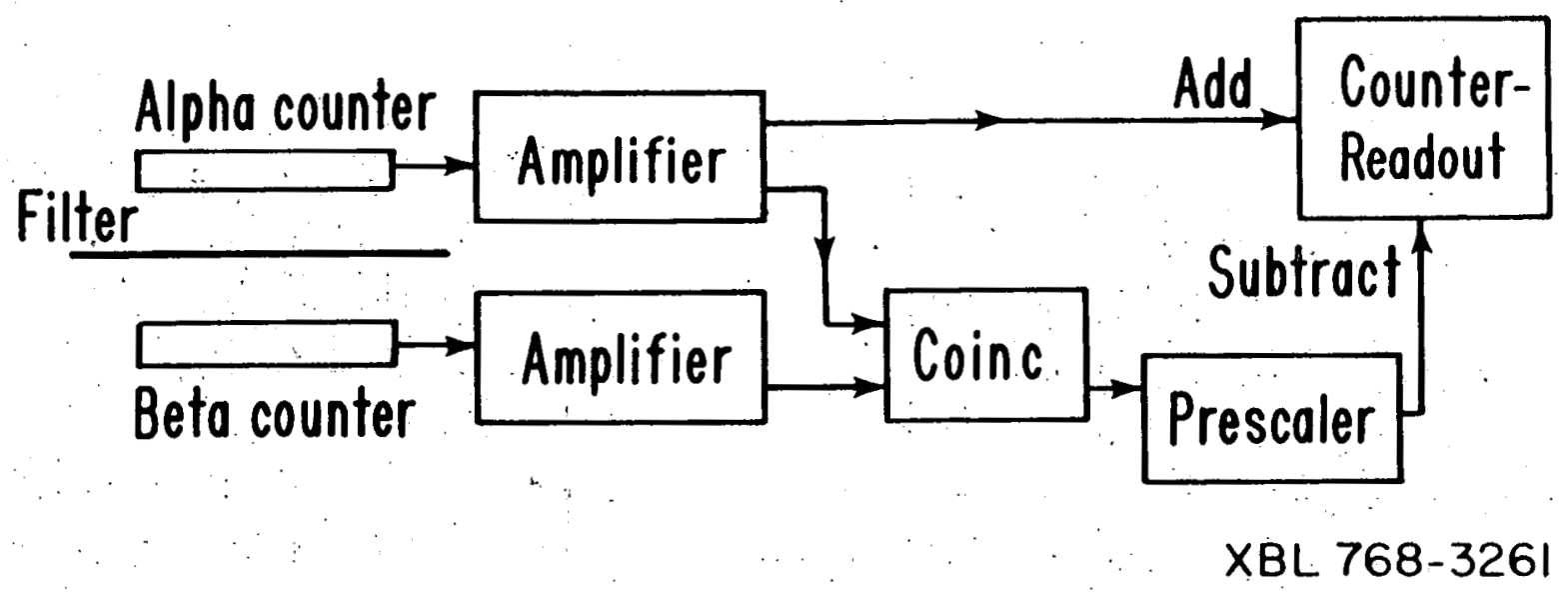

"Fig. 3-7. Schematic diagram of alpha counting system with associated-beta subtraction: 
3.2.1.3. Associated $\alpha-\beta$ Measurements

In the "associated $\beta$ " method, the alpha counts due to daughters of radon and thoron are determined by observation of the $B$ rays that effectively accompany the alpha decay of ${ }^{214} \mathrm{Po}\left(\mathrm{Ra} \mathrm{C}^{\prime}\right.$ ) and ${ }^{212} \mathrm{Po}$ (Th $\mathrm{C}^{\prime}$ ). Those nuclides are formed by $\beta$-decay and, because of their 164 and $0.3 \mu \mathrm{sec}$ respective half-1ives, almost immediately alpha decay. Observations of $\alpha-\beta$ coincidences can be used to estimate the radon and thoron daughter activities.

The basic system, shown schematically in Fig. 3-7 would consist of an alpha-sensitive and a beta-sensitive detector. Both detectors are counters, rather than spectroscopic instruments. The only discrimination against background activities is therefore from subtraction of the $\alpha-\beta$ coincidences, weighted to take account of the direct and coincidence efficiencies. This causes a number of possible difficulties:

a) If the long-lived $U$ or Pu activity is small compared with the radon/thoron activities, one will be subtracting two large numbers to obtain a small one; such subtraction may lead to substantial uncertainties.

b) Since the filter will be monitored by two different detectors with differing intrinsic and geometric detection efficiencies, the $\alpha-\beta$ coincidence rate must be electronically weighted before subtraction. Given fixed resolving times and detection efficiencies for the system, it is theoretically possible but not always achievable in practice, to cancel out both the radon and thoron chains exactly, aside from statistical fluctuations, provided the deposited daughters are in equilibrium, and the efficiencies are known.

c) 'This last consideration is non-trivial, since the betas to $\mathrm{Ra} \mathrm{C}^{\prime}$ have substantially different energy spectrum than those leading to Th $\mathrm{C}^{\prime}$, requiring care in compensating for the variation of detector efficiency with energy. This is especially important since for long collection periods, the thoron daughters may - because of their longer effective half-1ife (due to the 11 hour ${ }^{212} \mathrm{~Pb}$ ) dominate the shorter-lived radon daughters. In either case, careful measurement of the efficiencies is important because of the required subtraction. It must be emphasized, in any case, that the observed $\alpha-\beta$ coincidences are measures of the $\mathrm{Ra} \mathrm{C}^{\prime}$ and Th $\mathrm{C}^{\prime}$ activity. Subtraction from the total $\alpha$ rate depends on the assumption of equilibrium in the respective decay chains. Balance in any given chain, however, will depend on the relationship between the collection and counting periods. 
Instruments employing this technique have been built using a yariety of detector schemes. ${ }^{7-10}$ The alphas are typically counted by a scintillator-photomultiplier detector, the betas by a scintillation detector or a Geiger-Muller tube. The resulting instruments are typicaliy not as sensitive to the isotopes of interest as energy spectrometers. However, $\alpha-\beta$ subtraction may be incorporated in an alpha spectrometer, thereby improving the sensitivity over that associated with each of the individual techniques. However, perhaps due to the complexities in performing the subtraction reliably, this method is not often used for $U$ and Pu monitoring.

\subsubsection{Radiochemical Analysis and High Volume Air Sampling}

Another technique for air sampling is radiochemical analysis of the air filter, followed by electrodeposition of the radioisotopes and alpha spectroscopy. Prior to electrodeposition, the radioisotopes of interest must be brought into chemical solution; they can then be deposited onto a planchet (used as an electrode) by passage of an electric current through the solution. This technique is more difficult than the direct counting methods just described, since it requires chemical dissolution of the sample (along with the filter), followed by chemical extraction, then electrodeposition onto a planchet, before the actual alpha counting can take place. The details of these preparatory procedures are discussed in Section 3.2.2 under the category of water, soil, and biological sampling techniques. The primary purpose of these procedures is to remove the uranium and plutonium, or other elements of interest, from the medium in which they are contained and/or to separate the various radioisotopes from each other, thereby simplifying the counting analysis.

In the general technique of radiochemical analysis followed by alpha counting, the actuai detectors used for the counting are the same as are used in the methods discussed above. However, because of the greater intrinsic sensitivity available in the absence of, for example, radon daughters, the detector system may be arranged to take advantage of this situation. For example, no $\alpha-\beta$ subtraction is appropriate; nor are the particular window subtraction techniques associated with alpha spectroscopy in the presence of radon daughters necessary. The investment of time and effort to prepare the sample for spectroscopy often justifies the use of a more careful spectroscopic measurement, Counting chambers are often evacuated, thus eliminating energy degrading processes in air, to preserve the high intrinsic energy resolution of semiconductor detectors. Moreover, the total energy spectrum may be stored and analyzed in a more sophisticated manner than above. The result is sensitivity that approaches the theoretical maximum in the absence of competing backgrounds. Details are discussed below. 
It should be emphasized that this technique involves a commitment of time and equipment that is substantially more extensive then, for example, the commercially available, self-contained alpha spectroscopic units described above: Because of the difference in analysis time, the applicability of these distinct techniques differs considerably. For example, the radiochemical technique is more suited for long-term monitoring than for alarmed systems. And because of the investment in equipment and operating personnel, only a limited number of installations, such as major federal laboratories, would choose radiochemical preparation, except as an outside service purchased as a check on internal monitoring systems.

Environmental air monitoring with high-volume samplers is a technique which often uses radiochemical separation. These devices sample air at 10 to 50 cubic feet per minute, at least an order of magnitude faster than the alpha monitors discussed above. The latter instruments are primarily intended for occupational and source monitoring. In the general environment outside a nuclear facility (or at its fence), the activity concentrations are lower than at the facility itself, and the same is true of permissible concentrations. As a result, it is appropriate to sample at a higher volume. This usually requires larger filter areas, with typically 10 to 100 times the area used in low-volume samplers. Utilizing this entire filter area and obtaining the heightened sensitivity needed for environmental monitoring leads to a choice of radiochemical analysis prior to alpha spectroscopy. As suggested above, this technique requires a substantial analysis time, as we11 as notable investment in laboratory equipment and personnel time.

\section{2:2 Radiochemical Analysis of Water, Soil, and Biological Samples}

In many circumstances, the extent of a release or of a history of releases may be monitored by examination of environmental samples to measure deposition and/or uptake of radioisotopes. The most precise method of measuring the uranium and plutonium activity contained in such samples (or in the filter samples from air monitoring) is still alpha counting and/or spectroscopy. However, the activity cannot usually be measured while it is still in the sample matrix because of the short range of the 4 to $6 \mathrm{MeV}$ alpha particles associated with the important $U$ and $\mathrm{Pu}$ isotopes. Because of this short range, the maximum uranium mass thickness which may be monitored directly is a few $\mathrm{mg} / \mathrm{cm}^{2}$ which for most materials corresponds to a linear thickness on the order of 10 microns. "Móreover, any sample

*That is, the thickness $t=\mu / \rho$ ', where $u$ is the mass thickness and $\rho$ is the density. For a typical case, $\mu$ is a few $\mathrm{mg} / \mathrm{cm}^{3}$, and $\rho$ is a few $\mathrm{gm} / \mathrm{cm}^{3}$, so that $\mathrm{t} \simeq 10^{-3} \mathrm{~cm}$ or 10 microns. 
approaching this thickness will seriously degrade the energy resolution available. Other less sensitive techniques that are based on observation of $\gamma$ and $x$-rays do not suffer this difficulty as severely and are discussed under survey methods in Section 3.2.3.

The technique used for removing the mass of material which makes alpha measurements difficult is chemical separation to extract the elements of interest. Radiochemical analysis has the additional important benefit of ridding the sample of other elements whose activity would interfere with measurement of the activity being monitored. As a result, the detection scheme is usually alpha spectroscopy or even simple alpha counting, without auxiliary background suppression.

Chemical analysis may suffer from two difficulties aside from the need for a practical chemical separation procedure. One is the small amount of the radionuclide component of the sample. The other is the possible difficulty of bringing the radionuclide into solution as the first step in separation. These difficulties are particularly true of plutonium. For $239 \mathrm{Pu}, 1 \mathrm{pCi}$ has a mass of only $16 \mathrm{pg}$, and the weight of the same amount of activity of reactor grade plutonium is even smaller. As to solubility, the most common chemical form of plutonium is the dioxide $\left(\mathrm{PuO}_{2}\right)$, which can be extremely difficult to dissolve, ${ }^{11}$ particularly if it has been heated to high temperatures. Such firing may occur either as part of materials processing (such as in production of fuel pellets) or under conditions associated with accidental releases. To some extent, these difficulties may be alleviated by adding a "tracer" to the sample, i.e., a known quantity of: the same element; hopefully in a chemical form similar to that of the nuclide to be measured, but: with a different alpha spectrum. Assuming that a known amount of tracer can be brought into solution with the isotopes to be measured, the percentage yield of the tracer at the final detection device can be used to normalize the corresponding results for the isotopes of interest.

For radiochemical analysis of plutonium, the use of either ${ }^{236} \mathrm{Pu}$ or ${ }^{242} \mathrm{Pu}$ as tracer for chemical yield is common. These yield alpha groups which occur respectively, at 5.75 and $4.88 \mathrm{MeV}$, so that - using high resolution alpha spectroscopy, in the absence of radon daughter backgrounds - they may be easily distinguished from Pu 238, 234, and 240, the dominant activities in either weapons or reactor grade plutonium. ${ }^{242} \mathrm{Pu}$, with its low energy alpha, may be chosen to avoid possible contribution to background through energy degrading processes.

However, a tracer technique cannot account for insolubility losses, because there is no guarantee that equal proportions of the tracer and the sample activity 
will go into solution. This defeats the basic requirement on a tracer, that it chemically exchange with the "unknown" in the sample, ultimately giving a measure of yield. Another consideration is that enough tracer activity be used that counting statistics on the tracer groups do not substantially degrade the precision of the final result. 12

Two classes of chemical separation are used: total dissolution techniques, which bring the entire sample into solution, and leaching, which selectively extracts the substance of interest from the sample matrix. The yield often depends on which method is used (and indeed on the details of the individual methods) as we11 as on the solubility of the sample activity. Leaching is the less vigorous approach, hence more liable to error, and this technique may be checked by tota1dissolution. However, it should be noted that the efficacy of periodic checking will be reduced if the solubility of samples varies considerably. Moreover, care must be taken that even the total-dissolution method completely dissolves the species of interest. Although these comments are phrased to apply most directly to soil samples, the same general considerations apply to any type of macroscopic sample.

Returning to the question of air sampling, we may note that samples collected by filtering are often subjected to radiochemical analysis, in some cases actually preceded by direct alpha counting (for continuous monitoring purposes). Chemical treatment of an air filter differs only qualitatively from treatments of other sample types.

Samples are treated with acids, typically nitric plus some combination of hydrofluoric, hydrochloric, perchloric; yielding a solution out of which specific elements are extracted, often sequentially, by ion-exchange techniques. (See Ref. 13). Separated carrier solutions are then prepared for use in an electroplating technique, which deposits the radioisotope-bearing ions onto a planchet which may be observed in an alpha spectrometer. Alpha groups from each element may then be counted without background from other radioactive elements: Moreover, the planchet and detector system may be designed, by using an evacuated counting chamber, to achieve energy resolution of tens of $\mathrm{keV}$. Most often ${ }^{236} \mathrm{Pu}$ and $232 \mathrm{U}$ are used as tracers in this technique.

The treatment described above fits best into the "leaching" category. If the nuclides of interest are present in their more insoluble forms, high yield. may require complete dissolution of the sample. For many samples, this can be difficult. But $\mathrm{Sill}^{14}$ describes a method for soil samples which, after an initial: 
treatment with nitric and hydrofluoric acid, utilizes a high-temperature "fusion with anhydrous potassium fluoride" and subsequent "pyrosulfate fusion" to dissolve the sample completely. The resulting melt is treated, and various fractions separated (after barium sulfate precipitation), to yield solutions out of which (Th), (Pa, U, Np, and Pu), and (Am, Cu, Cf) fractions can be electrodeposited. Further, $U$ can be separated into its own fraction to preclude confusion with alphas from ${ }^{237} \mathrm{~Np}$. Another total dissolution method for measuring only plutonium has been described by Talvitie. ${ }^{15}$ In this procedure the sample is initially dissolved (if necessary) in hydrofluoric and nitric acid, then heated with hydrochloric acid, after which ion exchange is used to prepare a solution for electrodeposition.

The dissolution/fractionation step just described is only the first in the procedure for radiochemical analysis. (See Fig. 3-8) The next is invariably electrodeposition, which is not as complex as the chemical separations, but requires care to avoid loss of the radionuclides or contamination from residues of previous runs on the same equipment. For large initial samples, the chemical preparation method must be designed to segregate the interesting isotopes into a sma11 annount of material which, after deposition onto the planchet, does not degrade resolution in the alpha counting.

The final step in this procedure is alpha spectrometry. With an evacuated counting chamber and a solid state detector followed by the usual signal processing modules, leading into a multichannel analyzer, complete alpha spectra with resolution between 20 and $50 \mathrm{keV}$ may be accumulated. Provided care is taken to avoid buildup of background due to system contamination by processes such as implantation of recoil ions in the detector, interfering alpha groups such as those which cause difficulties under alpha spectrometry above are very strongly reduced. Because of this low background, positive identification of particular nuclides is possible with only a few counts in a peak.

Thus, for example, requiring only 5 counts in a peak that accounts for $50 \%$ of a nuclide's decay and using a sample preparation and spectroscopic system with $30 \%$ efficiency, $0.015 \mathrm{pCi}$ is the minimum detectable amount for a 1000 minute counting period. (A 1 hour air filter collection at 1 liter/sec assembles $0.20 \mathrm{pCi}$ of plutonium if it is present at the general public MPC level, for soluble $\mathrm{Pu}$, of $6 \times 10^{-17} \mathrm{Ci} / 1$.) Since counting periods greater than 1000 minutes are rarely useful, $0.01 \mathrm{pCi}$ represents a practical limit for sensitivity. By way of comparison, a continuous alpha monitor as described in Section 3.2.1.1. has a sensitivity greater than $1 \mathrm{MPC}$ - hr. (40 hour occupational), which corresponds to 


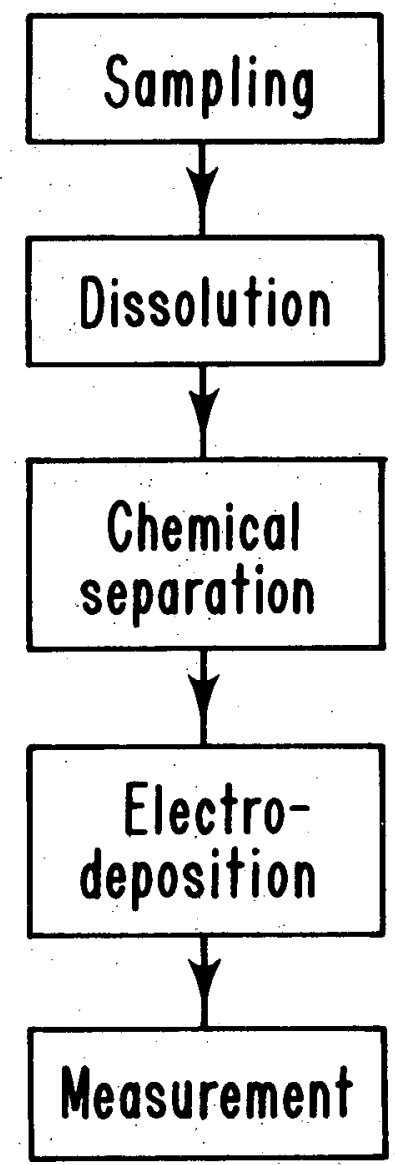

Air filter samples, soil samples, etc.

Total dissolution or leaching

A variety of chemical techniques

Deposit onto an appropriate substrate

Typically alpha measurement

XBL 768-3262

Fig. 3-8. Generalized flow diagram for radiochemical analysis. 
$8 \mathrm{pCi}$. The sensitivity is so much poorer than radiochemical analysis followed by alpha spectroscopy both because of limitätions on collection/counting periods and because of alpha backgrounds whose contributions cannot be subtracted exactly.

For air-monitoring applications, the $0.01 \mathrm{pCi}$ limit is still significant as compared with maximum permissible concentrations. For measurements in other media, it does not represent a limit. For example, the genera1 public MPC for plutonium and uranium in water are 50,000 and 200,000 pCi/1iter, respectively. These are relatively large amounts compared with instrumental sensitivities. However, the ability to monitor water and other samples to lower concentrations is still an important requirement for measuring releases of radionuclides.

The disadvantages of radiochemical analysis are the time, equipment, and personne1 required. Analysis requires at least one day after collection and involves equipment for chemical dissolution, ion exchange, electrodeposition, and spectroscopy. Skilled workers are an absnlute necessity.

\subsubsection{Methods for. Field Survey and Personne1 Monitoring}

In many circumstances, a relatively rapid determination of the radionuclide content of a sample or an area is needed. This precludes complex chemical analysis or other treatment, particularly if what is required is the survey of a living person or a large land area. Under such conditions, measurement of radionuclide distributions via alpha particles is impractical, except at very high concentrations, because of the short range of the particles through matter and/or degradation of the alpha energy. Electromagnetic radiation is a much more suitable signature when significant amounts of material exist between radionuclide and detector. For both uranium and plutonium, concentrations may be monitored by $\dot{x}$-rays or $\gamma$-rays emitted immediately after alpha decay. The $x$-rays will be characteristic primarily of the element decaying, the $\gamma$-rays of the particular isotope.

Survey instruments for plutonium often make use of $\mathrm{L} \cdot \mathrm{x}$-rays from the uranium atom remaining after alpha decay. The most prominent lines occur at 13.6, 17.2, and $20: 2, \mathrm{keV}$ with relative intensities 100,120, and $25^{16}$ respectively. Instruments using this technique are usually intended primarily for monitoring of weapons grade Pu. However, $x$-ray spectra should be relatively independent of the particular isotope, excepting ${ }^{241} \mathrm{Pu}$, so that reactor grade plutionium could be measured equally we11. ${ }^{241} \mathrm{Pu}$ beta decays, but its daughter, ${ }^{241} \mathrm{Am}$, has its own characteristic $x$-ray 1 ines $(14.0 ; 17.820 .8,26.3 \mathrm{keV}$ with branches of $12 \%, 13 \%$, $3 \%$, and $2.5 \%$ intensity, respectively) ${ }^{17}$ which may be used for plutonium monitoring, 
Table 3-5. Principal gamma rays following decay of uranium and plutonium (from Ref. 2)

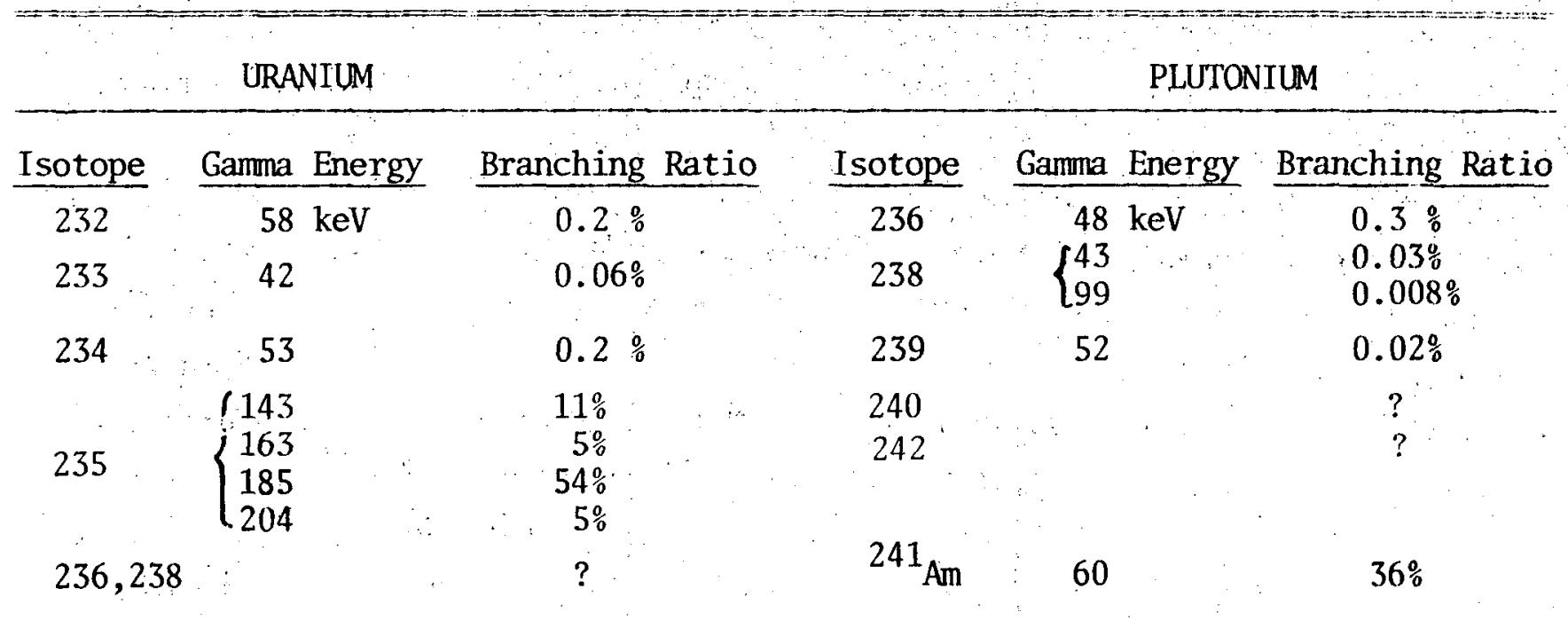

provided the "age" of the plutonium is known" and the americium has not becone chemically separated. X-ray spectroscopy may in principle be used for uranium, which yields thorium $L$-rays at $13.0,16.0$, and $19.2 \mathrm{keV}, 18$ but this has not often been done.

Individual isotopes have characteristic gamma rays which follow alpha decay to excited states in daughter nuclei (See Table 3-4). For the most important isotopes of uranium and plutonium, the principal lines (in keV), with their intensities relative to the total disintegration rate, are given in Table 3-5.

Few strong gamma rays are associated with the above isotopes. For most practical measurement applications in the nuclear fuel cycle, though, monitoring via gamma rays must depend on these lines or - in a few cases - on those of immediate daughters, such as ${ }^{241} \mathrm{Am}$. The one exception is that uranium occurring naturally in environmental media may be measured by observation of gamma rays from relatively distant daughters. The most useful gamma ray in this category is the ${ }^{214} \mathrm{Bi}$ line at $1.76 \mathrm{MeV}$. However, the fact that ${ }^{214} \mathrm{Bi}$ occurs after radon requires that the possibility of lack of equilibrium be seriously considered. Techniques depending on daughter activities, however, are used more often for assays of uranium-bearing ore, an application of little interest here, than for monitoring of releases from nuclear facilities.

* That is, one must know what percentage of the ${ }^{241} \mathrm{Pu}$ has transformed to ${ }^{241} \mathrm{Am}$. 
Returning to the observation of $x$ - and $\gamma$-rays directly from uranium and plutonium, we direct our attention to the two major classes of detectors: scintillation counters of relatively low resolution and semiconductor detectors with substantially higher resolution. The first are most often NaI(T1) detectors coupled to photomultipliers, the second Ge(Li) detectors, connected to charge sensitive amplifiers. Two other potentially important subsets of the semiconductor class are the intrinsic germanium detector, which has to be cooled only while it is operating, and the CdTe detector, which can be operated without a cryostat.

All of these detectors may be applied for a large range of photon energies from $\mathrm{keV}$ to $\mathrm{MeV}$. For the present purposes, though, they are usually designed to maximize sensitivity at low energies, from 10 to, at most, a few hundred $\mathrm{keV}$. Thin detectors are intentionally chosen to minimize the background caused by scattered, but incompletely absorbed, higher-energy photons. Other important considerations for survey instruments are: ${ }^{19}$

1. The effective aperture of the detector: for surveys of low concentrations, a large aperture is needed in order to obtain sufficiently high counting rates.

2. Energy resolution: the choice of thin detectors may also degrade efficiency and/or resolution for photons of interest.

3. Dependence of the radiation field on angle: for radioactivity in soils, a large portion of the radiation from land areas is emitted at large angles with respect to the vertical.

4. Probability of $x$-ray "escape": one would like to minimize background caused by events in which the incident photon ejects a photo-electron and the subsequent $x$-ray escapes from the detector. (This may be accomplished by choice of low atomic number materials for the detector, but this reduces the stopping power.)

One common type of area survey instrument is designed around a thin NaI(T1) crystal of large area. The most straightforward version of such an instrument couples a photomultiplier to the crystal, allowing energy analysis of radiation interacting with the crystal. The archetype of this instrument, designed for monitoring of plutonium contamination by observation of americium photons is the FIDLER, ${ }^{20}$ which has a $5^{\prime \prime}$ diameter, 1/16" thick crysta1. This is a portable instrument with energy windows around 17 and $60 \mathrm{keV}$, the principal $x$-ray and $\gamma$-ray groups from ${ }^{241}$ Am. (See Fig. 3-9). Such a planar detector can average over a large area. Interpretation of results will depend on this averaging, on the isotopic composition of the contanination, and on its depth distribution in the soil. 


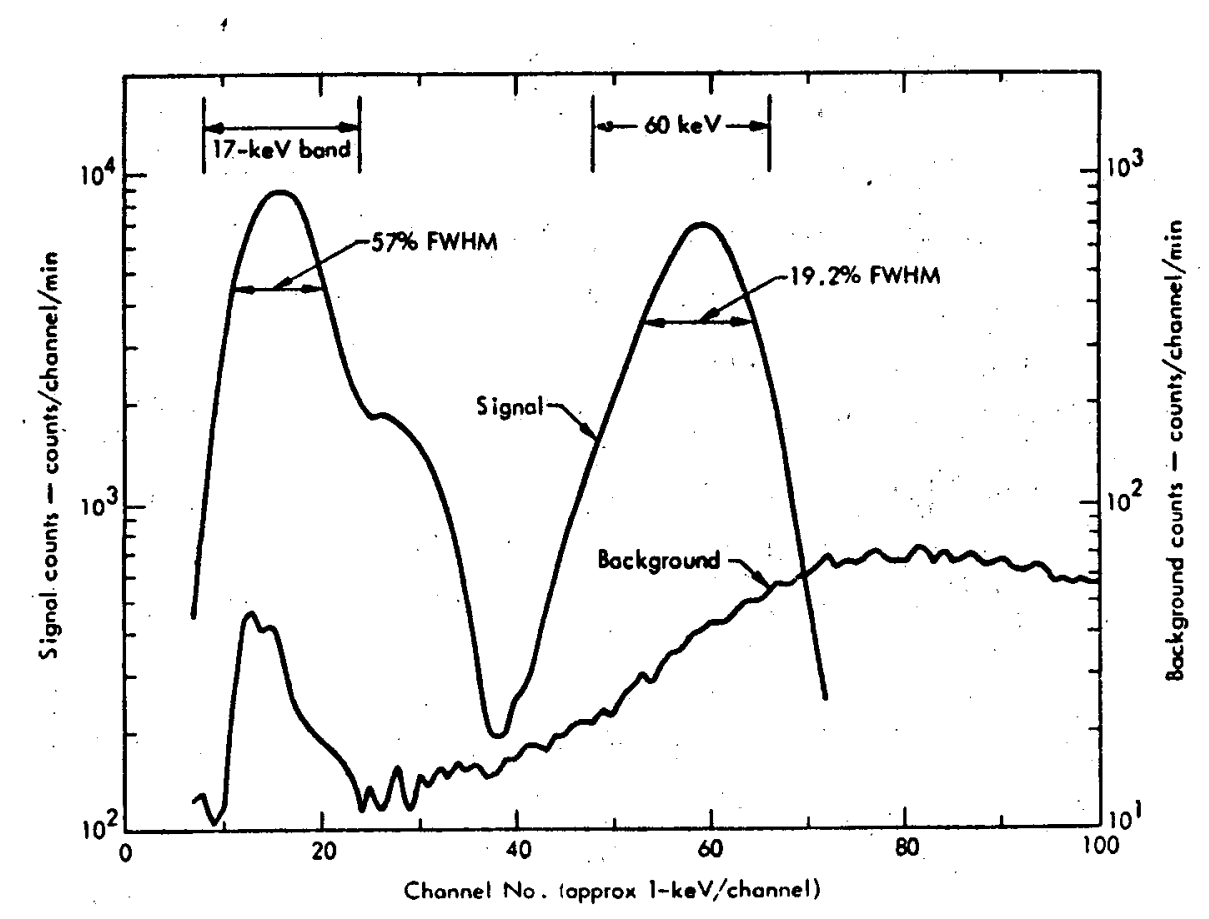

Fig. 3-9. Spectra for ${ }^{241} \mathrm{Am}$ and background as measured with a 5-in.-diam by 1/16-in.-thick NaI(T1) scintillation detector. Optimum discriminator settings for $17-\mathrm{keV}$ band and $60 \mathrm{keV}$ are shown (from Ref. 20). 
Both of the last two will depend on the "age" of the contamination. This especially affects interpretation of the results due to the fact the monitoring is via ${ }^{241} \mathrm{Am}$. Since this is the daughter of ${ }^{241} \mathrm{Pu}$, which has a 13 year half-1ife, isotopic composition will change relatively rapidiy. It will also depend on the isotopic mixture of the plutonium. Typical minimum detectable ${ }^{241}$ Am from a distributed source is $15,000 \mathrm{pCi} / \mathrm{m}^{2}$.

Similar instruments have been developed, based on other scintillators, such as $\mathrm{CaF}_{2}(\mathrm{Eu}) .21$ The possibility of developing an area-survey "phoswich" instrument (see below) to discriminate against background from high-energy photons is being considered. 22

Recent work on soil survey techniques has included use of Ge(Li) detectors, with their much superior energy resolution, which reduces the amount of background under the peak(s) of interest. Straightforward volume detectors, ${ }^{23}$ using the ${ }^{241}$ Am line, yield a sensitivity that is somewhat superior to the FIDLER. A more sophisticated development is the use of an array of large-area but thin (2.5 $\mathrm{mm})$ $\mathrm{Ge}(\mathrm{Li})$ detectors; as above, choosing a thin detector reduces the background due to partially-absorbed high-energy photons. The sensitivity of the latter instrument 24 is said to be roughly a factor of 10 better than that of the FIDLER. On the other hand, Ge(Li) detectors must be continually cooled; the necessary cryostat, and also the more complete data analysis required for this improvement in sensitivity, preclude designing such a detector into a portable instrument. It is, however, suited for use with a van.

The instruments just described were developed for use in surveys of contamination due to plutonium, particularly of weapons grade. They are not suitable for observation of uranium $\gamma$-rays, with the exception of the large-volume Ge(Li) counter, which is sensitive to high-energy photons from daughters. See for example the ${ }^{214} \mathrm{Bi}$ line in Fig. 3-10 from Ref. 25. Because of these high-energy photons, $\mathrm{NaI}$ (T1) detectors used for uranium monitoring would be chosen to be approximately a centimeter thick. Such detectors have sensitivity in monitoring natural uranium on the order of $1 \mathrm{ppm}$. However, for monitoring of either fresh or spent fuel, daughters will for the most part not be present, so that detection must depend on the decay of the actual uranium isotope. The only $\gamma$-rays of significant strength are from ${ }^{235} \mathrm{U}$, and the strongest of these (185 keV) occurs at the same energy, within typical detector resolution, as a ${ }^{226} \mathrm{Ra} \gamma$-ray. Survey instmments for other important isotopes (such as. ${ }^{232} U$ and ${ }^{233} U$ ) must depend on extremely thin detectors, sensitive to the $\mathrm{L} x$-rays from uranium decay, or possibly on detectors sensitive to the high energy ( $2 \mathrm{MeV}$ ) photon from the ${ }^{208} \mathrm{Tl}$ daughter of ${ }^{232} \mathrm{U}$. 

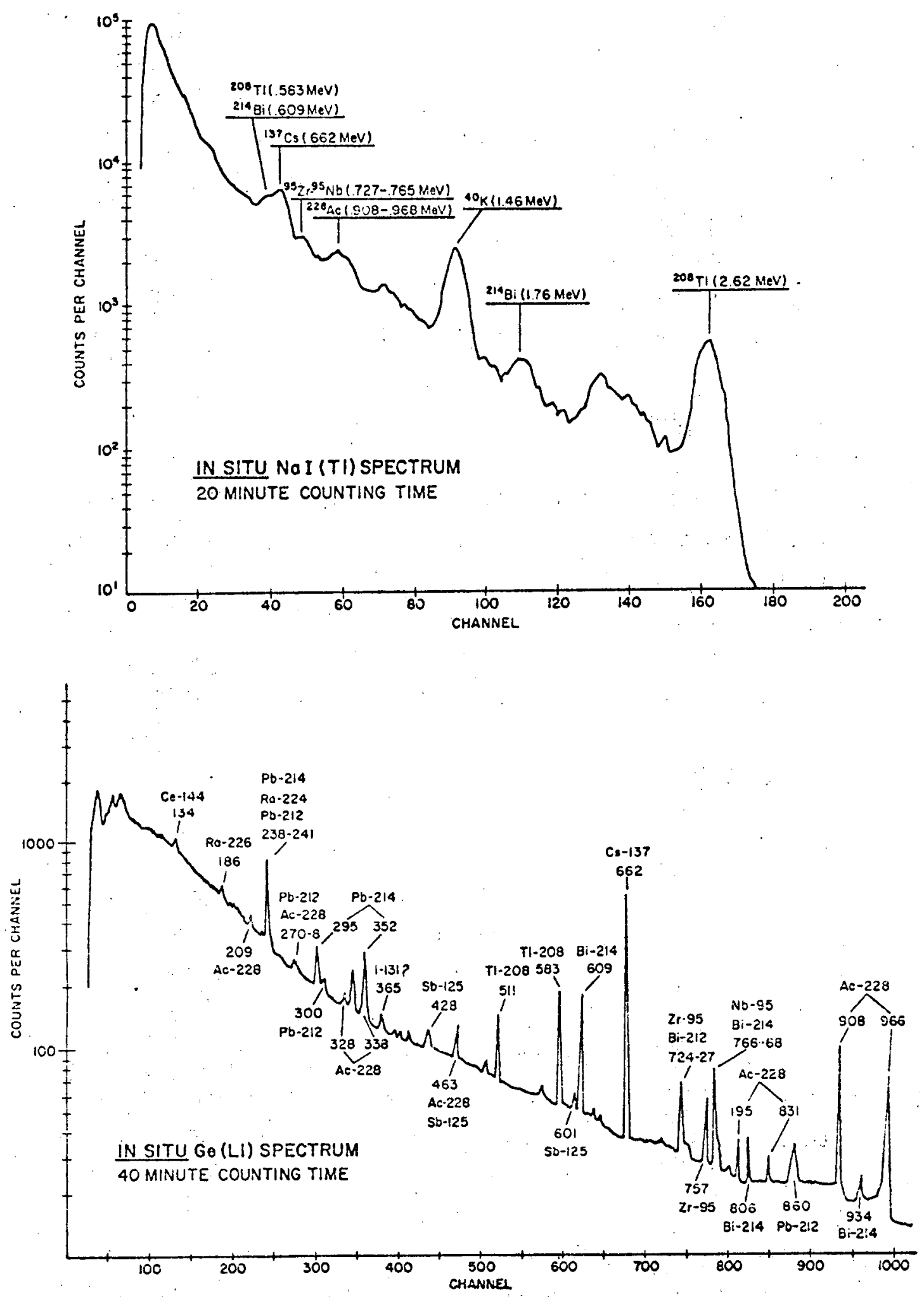

Fig. 3-10. Upper drawing: in situ spectrum northeastern U.S. location, taken with $10 \mathrm{~cm} \times 10 \mathrm{~cm} \mathrm{NaI}$ (T1) crystal, 20-minute counting time. Lower drawing: in situ spectrum (photon energies in $\mathrm{keV}$ ), at same location, but with $60-\mathrm{cm}^{3}$ $\mathrm{Ge}(\mathrm{Li})$ detector, 40 minute counting time. (From Ref. 25). 
A more recent advance in solid state instrumentation is the development of detectors which require cooling only at the time of use or not at all. An example of the former is the intrinsic germanium detector, which therefore has a somewhat wider range of application than Ge(Li). An example of the latter is CdTe, a material which can be used at room temperature, but whose resolution is intermediate between $\mathrm{NaI}(\mathrm{Tl})$ and $\mathrm{Ge}(\mathrm{Li}){ }^{26}$ Both intrinsic germanium and room temperature materials, however, are not usually applied to environmental surveys but to assay of fuel materials, an application in which the count rate due to $U$ or Pu would be expected to be high.

The requirements on measurements for monitoring of human subjects differ somewhat from those for area survey. Fixed installations are often possible, and the backgrounds are better defined.: The manner in which NaI(T1) is normally used. is in a "phoswich" detector, composed of a thin NaI(T1) detector backed by a thick CsI(T1) detector, which stops high energy photons. (See Fig. 3-11). The latter scintillator has a longer decay time that NaI(T1), so that its signal may be used to suppress background in the thin detector from high-energy photons. Sensitivity can therefore be improved over the roughly $6000 \mathrm{pCi}$ lung burden that could be detected (for a 100 minute counting period) by a thin NaI(T1) detector without suppression. Other workers have used gas proportional counters, ${ }^{16} \mathrm{Ge}(\mathrm{Li})$ counters, ${ }^{27} \mathrm{Si}(\mathrm{Li})$ detectors, ${ }^{28}$ and intrinsic germanium ${ }^{29}$ for 1 ung and/or wound assay. These are invariably applied to possible cases of plutonium contamination, and not uranium. In either case, such instruments belong to the general class of personne1 rather than release-monitoring devices. In addition, a variety of relatively insensitive instruments, usually not specific to either uranium or plutonium, may be used for checking for radioactive contamination of workers or their clothing.

With the exception of instruments for uranium ore assay and search, most of these instruments are not commerically available. The one notable exception is the phoswich detector. Use of this device requires careful set-up and calibration because of its timing-based anti-coincidence circuitry. Specialized survey instruments do not usually constitute part of a routine monitoring program, except at special sites, such as the Nevada Test Site and other federal testing and/or production facilities. As discussed in a later section, such instrumentation might profitably be available on an "as-needed" or emergency basis for surveying in the vicinity of suspected release points. 


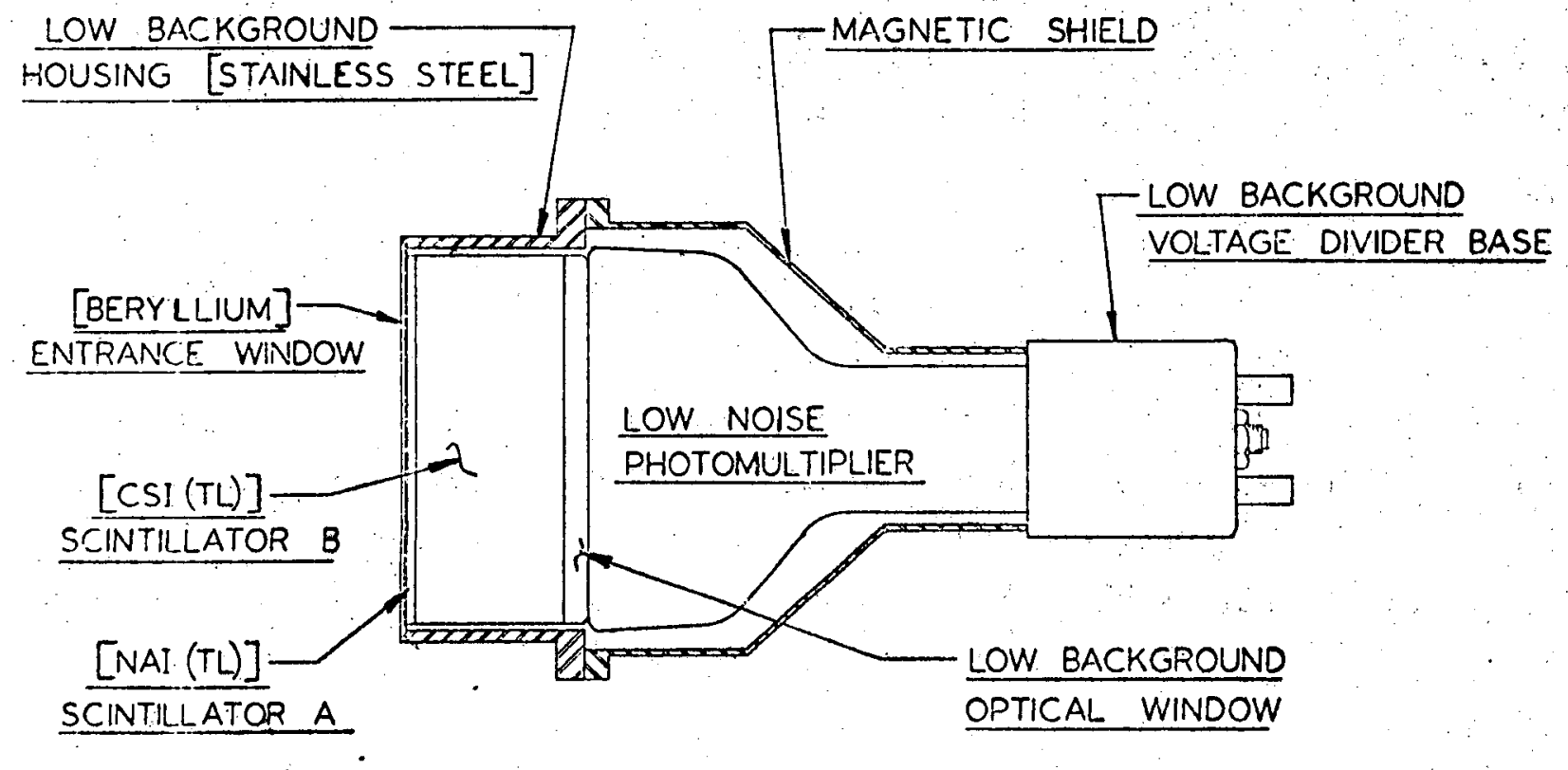

Fig. 3-11. Harshaw Phoswich detector: matched window type of $x$-ray and low energy gamma detection. 


\subsubsection{Track-etch Technique}

Alpha particles from decay of isotopes of interest may be recorded in a permanent fashion in the bulk of some materials. The particles produce ionization along their paths and the material can be treated subsequently to make tracks visible. These tracks can then be observed with the help of a microscope.

This same basic technique may be used for fission track monitoring, discussed below in Section 3.3.1.2.

3.3 Monitoring via Induced Activities

Having considered those monitoring techniques which depend on radiation resulting from the spontaneous radioactive decay of the isotopes of interest, we may now turn to other methods for identifying uranium and plutonium. These other methods may be based on induced (as opposed to spontaneous) activity, mass, or chemistry. These techniques will not depend directly on spontaneous decay, but maximum permissible concentrations and related standards are tied directly to such decay. As a genera1 rule we may expect that materials which have a high specific activity (decay rate per unit mass) will be more difficult to measure at the required minimum levels by non-decay techniques than materials with low specific activities. For the latter, a standard expressed in terms of some maximum activity will translate into a relatively large number of atoms present, thus making monitoring via non-decay techniques more practical.

The first class of non-decay techniques which we will consider produces a signature for the isotopes of interest by inducing such material to give off (or, in principle, absorb) radiation. This radiation may be associated with either the nucleus or the electronic structure. (That is, it may be either nuclear or atomic radiation.) A distinct advantage to monitoring via radiation is the possibility of detecting the isotopes of interest even when embedded in some matrix: radiation characteristic of these isotopes can transverse such material to reach a detector. However, this advantage is also enjoyed by some techniques discussed under decay monitoring. Monitoring via induced activities can only be favored where the technique offers sensitivity or ease of application that is superior to decay-monitoring techniques.

Three important classes of induced activities will be described below:

1. Neutron-induced fission - applicable to fissile isotopes

2. Activation analysis - always applicable, in principle; yields radiation characteristic of individual isotopes

3. X-ray fluorescence - gives a signature characteristic of an element rather than of a particular isotope. 


\subsubsection{Neutron-Induced Fission}

Any mixture of uranium or plutonium will contain a substantial portion of fissile material, so that - in principle - presence of these elements may be indicated by observation of fission induced by slow neutrons. * Fission may be indicated in a number of ways, the most important of which are delayed neutrons and fission tracks. In either case, the setup required for monitoring is more extensive than that associated with more direct techniques, such as alpha monitoring, especially since a reactor or other substantial source of neutrons is needed. Because this implies analysis at a location remote from collection, analysis of samples cannot typically be accomplished rapidly. This fact further limits application of such techniques.

\subsubsection{Delayed Neutrons}

When a uranium or plutonium nucleus undergoes fission, approximately two neutrons, on the average, are released. Although almost a11 of these are freed instantaneously, 0.3 to $0.5 \%$ of them result from neutron decay of fission product daughters, so that their appearance is delayed by the time taken for production of these daughters via beta decay. These delayed neutrons can be detected by various types of systems, such as a plastic scintillator mounted on a photomultiplier. Delayed neutron emission is an excellent signature for fission, since the only other known neutron-decaying nuclides are nitrogen 17 (with a 4.2 second half-life) and lithium 9 (0.17 second). Contribution of these two nuclides to background can be elininated by allowing them to decay away after irradiation of a sample and before counting begins. Observation of decayed neutrons has the distinct advantage that it can be used for bulk samples, because of the long range of neutrons. As a result, relatively sma11 concentrations in soil and other samples may be detected. For ordinary uranium samples, and with neutron fluxes normally available from a reactor, the counts obtained are due principally to ${ }^{235_{U}}$. The sensitivity limit for natural uranium is on the order of 0.1 to 0.01 micrograms 30,31 as the technique is usually applied. For a bulk sample, say of 10 grams, this corresponds to a concentration limit of $3 \mathrm{ppb}$. This is roughly the average concentration of uranium in the earth's crust. It is interesting to note that 0.01 micrograms or $10 \mathrm{ng}$ is also the present limit on detectability of heavy elements on air filters analyzed

\footnotetext{
${ }^{*}$ Fast neutrons can induce a larger number of isotopes, including those of thorium, to fission. However, the fission cross sections at high energy are not as large. Slow neutrons are more often used.
} 
by $x$-ray fluorescence. (See Section 3.3.3.) Note that for natural uranium $10 \mathrm{ng}$ corresponds to about $3 \times 10^{-15} \mathrm{Ci}$, a very sma11 activity. For isotopes with a much shorter half-life (such as ${ }^{239} \mathrm{Pu}$ or ${ }^{233} \mathrm{U}$ ), the minimum detectable activity is $10^{4}$ times larger.

\subsubsection{Fission Track-etch Techniques}

A much more sensitive technique for detection of fission events is observation of the ionization tracks left by the stopping of the fission fragments. Uranium bearing material is placed on a thin sheet of plastic (e.g., polycarbonate ${ }^{30}$ ) which is placed in the neutron flux from a reactor. After storage to allow for decay of short-lived activities, the polycarbonate is etched in an $\mathrm{NaOH}$ solution, then examined under a microscope. Sensitivities down to $10^{-14} \mathrm{~g}$ of natural uranium are reported. ${ }^{30}$ Presumably comparable amounts of other fissile material could be detected, although it would not be possible to distinguish one fissile material from another. In any case, this appears to be the most sensitive method presently available for detection of fissile material. The method does require that the sample be deposited as a very thin layer, so that fission fragments can escape into the detector material. For the case of an air filter it is often possible to place this directly on the detector. As a result, this method would appear to be as convenient for the detection of very small amounts of material collected by air filters as any other available technique. Disadvantages are that it needs a neutron source, such as a reactor, and requires a long delay between collection and analysis.

\subsubsection{Neutron Activation Analysis}

In neutron activation techniques, the sample is placed in a flux of neutrons, and nuclei in the sample capture neutrons to form radioactive nuclei. Decay of any particular type of nuclide will lead to characteristic gamma rays, which can be used to identify the nuclide and ultimately - when enough such types are identified - the nuclide from which it was formed. This technique may be used for identification of uranium and plutonium. However, probably because of the need for a neutron source in any case, and because detection of neutron-induced fission is a more sensitive technique, it is not often used for uranium and plutonium.

\subsubsection{X-Ray Fluorescence}

If electromagnetic radiation with energy greater than that of the $x$-ray emission lines strikes an atom, electrons from one of the deep she11s may be excited. As the atom returns to its ground state, $x$-radiation characteristic of electronic transitions in these deep she11s will be emitted. This radiation, commonly called 
fluorescence, may be used as a signature for measurement of trace quantities of elements in environmental samples. 32,33 In the case of air filter analysis, the limit of detection for heavy elements is about $10 \mathrm{ng} / \mathrm{cm}^{2}$, a sensitivity limit which is comparable to that of some of the techniques listed above for natural uranium (in particular delayed fission neutron detection of uranium). This 10 $\mathrm{ng} / \mathrm{cm}^{2}$. corresponds, for a filter area of a few $\mathrm{cm}^{2}$, to about $3 \times 10^{-15} \mathrm{Ci}$. of uranium, a sensitivity that is much superior to decay monitoring techniques. However for short-lived activities, implying a high specific activity, the $10 \mathrm{ng}$ limit corresponds to a relatively large amount of activity; for example it corresponds to about $1 \mathrm{nCi}$ of ${ }^{239} \mathrm{Pu}$.

Since most of the present effort in $x$-ray fluorescence has been directed toward detection of the lighter elements, it is not clear what improvement might be realized for heavy elements if techniques were optimized for these elements. A possible difficulty is interference from lines due to other elements in the sample. If such interference required the utilization of chemical separation techniques, it would clearly reduce the attractiveness of the $x$-ray fluorescence method since one of its salient features is that air filters may be analyzed directly and automatically, using only short measurement periods. One might ask whether it might be useful to apply $x$-ray fluorescence in circumstances where chemical separation must be used in any case. Then the fraction of high specific activity elements might be measured by conventional alpha monitoring, whereas the low specific activity fraction could be measured by $x$-ray fluorescence, with its short counting time. For environmental monitoring, there is an advantage to measuring the rather low baseline concentrations of nuclides of interest. such as uranium and thorium. Changes in their low levels would then indicate some abnormality. It may be possible to deposit the chemically extracted material on a matrix which, when used with the fluorescence technique, reduces the minimum detectable amount below $10 \mathrm{ng} / \mathrm{cm}^{2}$. An important factor in the existing sensitivity limit is scattering from the filter material. Deposition on a thinner matrix would presumably reduce this problem. X-ray fluorescence can be applied to any. activity extracted chemically, whether the initial matrix was an air filter, soil, or other sample.

Electrons may also be excited by other than x-rays, although this is the technique most often used. Other possibilities are a proton beam or an electron beam. Electron beams are sometimes chosen in more specialized applications. For example, an electron-excited x-ray probe may be used to analyze the elemental 
content of small particles collected on an air filter. Such a technique, however, has a much narrower range of application that the more general approach to trace element analysis mentioned above.

In general, it is important to note that $x$-ray fluorescence analysis identifies elements, rather than isotopes.

3.4 Monitoring Using Mas̀s Spectroscopy

Another physical characteristic which may be utilized for monitoring is the atomic mass of the substance of interest. The manner in which this mass is measured in a mass spectrographic method is to ionize the atoms in the sample, accelerate them to some specified energy, then allow them to pass through a magnetic field. Atoms (or molecules) of differing mass will be deflected through different angles and will be collected at different points along the detector. One of the more difficult aspects of this approach is to ionize the atoms in a manner that is consistent with the chemical form or physical size of the sample and with the requirements of the acceleration and magnetic analysis system. Another important goal is that a significant portion of the ions entering the analysis system reach effective areas of the collector or detector. To achieve this the magnets are typically designed to have advantageous focussing properties.

Mass spectroscopy is not often emphasized for monitoring radioactive nuclides, since the required equipment is bulky and often difficult to use. Nor has the technique offered any clear advantages over more convenient methods. Generally the sample to be observed has to be subjected to complex chemical preparation before it can be introduced into the ion source. Once this is done, the substantially easier and less costly alpha monitoring method may be used. However, it should be noted that in principle a mass spectrographic technique can be more sensitive than any decay monitoring technique, primarily because the mass analysis does not require the nucleus to decay in order to be observed. In this respect, mass analysis could also be much more sensitive than methods that induce activity, because the latter approach usually has a relatively poor efficiency for exciting the atom or nucleus.

For analysis of soil or other macroscopic samples, chemical preparation is inevitable to bring the sample into a form that is suitable for ionization. However, for the single case most important for monitoring of releases, air monitoring, the amount of solid material to be dealt with is relatively small. Moreover, the form in which uranium or plutonium would normally be present in the air is in the form of particulates, rather than as a gas. However, mass analysis of the activity collected on air filters would still require chemical preconcentration. Because 
the mass analysis could then proceed very rapidly, there may be circumstances in which a mass analysis system designed for this case may be advantageous.

The most clearly advantageous system would somehow introduce the airborne activity directly into the ion source, effectively bypassing any preparation process. No such system is presently available, but work on its development is progressing. 34 Because the activity of interest is carried in particulate form, one can use the momentum of these particles, causing them to impact against a surface, to collect the activity from the much larger volume (and mass) of air. Since in principle that surface can be designed to act as the filament in a mass spectrograph ion source, one will have placed the activity in a position suitable for analysis. The fact that such an ion source must operate at very low pressure effectively limits the rate at which air can be sampled. This is a serious disadvantage if the activity is of interest is not we11 distributed throughout space. (See section 3.6.2 below, where a possible solution to this problem is discussed.) On the other hand, the low operating pressure in the chamber makes it possible to have even very small particulates impinge on the filament. This avoids loss of very sma11 particulates ( $\leq 0.5$ micron diameter) that would pass through the impacting stages of a device operating at atmospheric pressure. (See Section 3.6.2.)

Table 3-6 shows possible sensitivities from alpha spectroscopy, $x$-ray fluorescence, and mass spectroscopy; al1 assuming 1 liter/sec collection and specified minimum detectable amounts.

\subsection{Other Monitoring Techniques}

A variety of techniques that are less sensitive than those discussed above may be used for measurement of higher concentrations or larger amounts of uranium and/or plutonium. These methods are typically based on preparatory chemical analysis followed by either electrochemical or photometric measurement. Into the first category fall coulometry, ${ }^{35,36}$ polography, ${ }^{37}$ amperometric titration, and others. The second category includes fluorometry ${ }^{13}$ and general spectrophotometry (including $x$-ray fluorescence, which was treated separately above because of its relatively good sensitivity). Many of these techniques give accurate results,. but require large amounts of the substance of interest. Because release monitoring usually involves sma11 amounts of material, we sha11 not discuss these methods. Reference 13 includes discussion of methods involving relatively large material quantities. 
Table 3-6. Limits on sensitivity for plutonium and uranium measurement (given in general public MPC-hours, ${ }^{a}$ assuming air sampling at 1 liter/second)

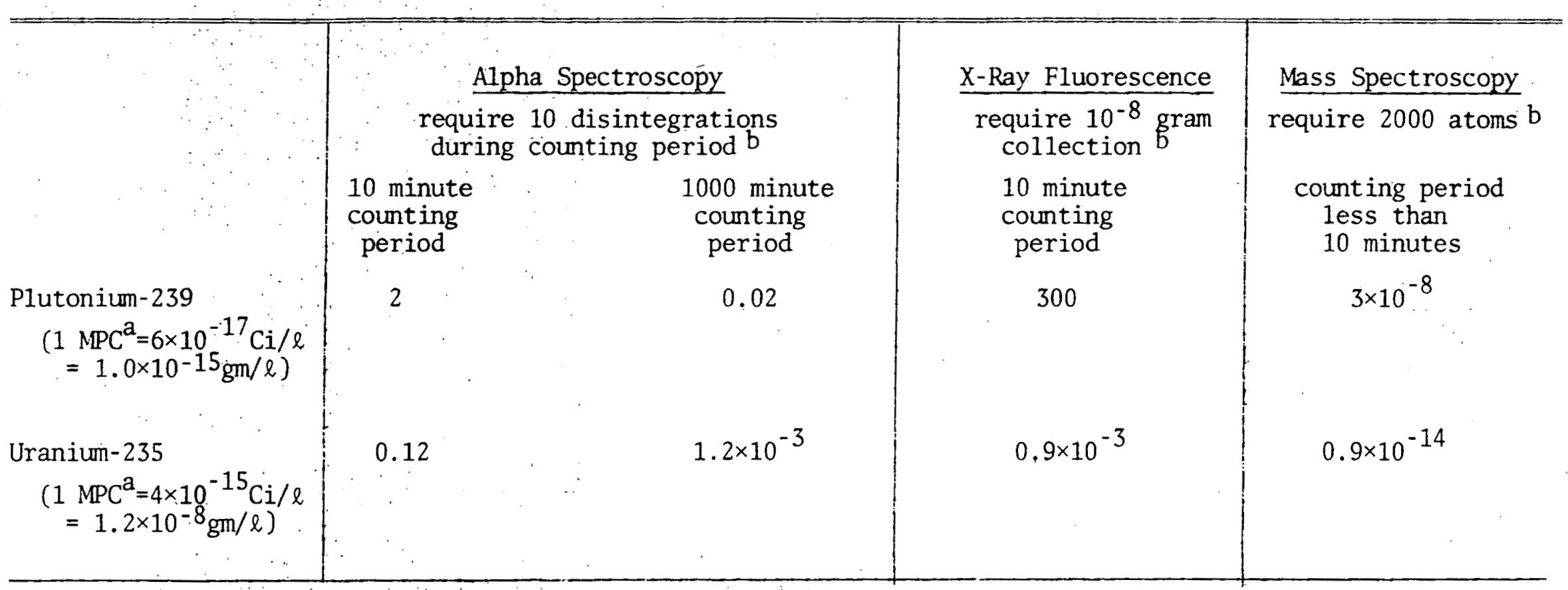

${ }^{a}$ A general public maximm permissible concentration is one tenth the smallest value given in Table 2-2.

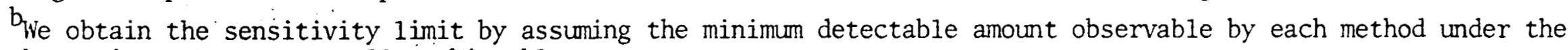
best circumstances normally achievable. 
3.6 Associated Radiochemical and Physical Techniques.

A number of important chemical and/or physical procedures are implicit in the specific monitoring methods discussed above. The details of these procedures or techniques have a substantial bearing on the effectiveness of monitoring. Of particular importance are the questions of chemical analysis and sample collection or particle sizing.

3.6.1 Chemistry

As was apparent in the previous sections, many monitoring techniques require chemical separation and even, in the less sensitive methods, chemical measurement. The most sensitive methods currently available require substantial chemical preparation of a sample; in contrast to this, the fastest (albeit less sensitive) methods completely avoid such preparation. This general observation, that for the most sensitive methods, time-consuming chemical procedures are required, seems inevitable except in cases where the substance of interest is not contained in a large amount of material. One such case is air monitoring. * Even in this case, the trade-off between speed and sensitivity usually exists.

Air monitoring offers the one hope of combining rapid measurement with high sensitivity, since rather small samples by mass are collected. However, sensitivity is impaired by the possible presence of large backgrounds, whose effect must somehow be removed or reduced. Complete removal prior to decay monitoring does not appear possible except via chemistry. The alternative is to use methods based on induced activities or possibly mass differences, as discussed in Sections 3.3 and 3.4. However, neither of these methods has yet combined good sensitivity with rapid or convenient monitoring capability. The greatest sensitivity currently available requires either chemical separation or neutron generators. 3.6.2 Size and Collection

An important air monitoring consideration, which we have not mentioned above except in the case of mass spectroscopy, is the size distribution for particles carrying the activity of interest. The size distribution of particles carrying other activities is also of concern if these activities constitute a background interfering with measurement of the activities under consideration here.

Particle size is important in two distinct respects; 1) it affects the release and transport of associated radioactivity and the extent to which this radioactivity can subject humans (or other organisms) to exposure, and

* Water monitoring may be another since water can be easily evaporated. 
2) it influences the effectiveness of any monitoring technique, A crucial consideration is that the size of particles most easily collected by the pulmonary region is in the range 0,1 to 5 microns. Particles much smaller or much larger than this range are not as easily retained.

Mechanica1, chemical, and accidental release mechanisms associated with the nuclear fuel cycle can produce particles in the 0.1 to 5 micron range. Chemical processes usua11y produce the smaller sizes, mechanical the larger; accidentally produced particles are not so easily characterized. A number of studies have dealt with the question of the size distribution of particles emitted from plutonium processing facilities. The most extensive of these studies included measurements of particles sizes produced at a number of different AEC facilities. ${ }^{38}$ In Fig. 3-12, it is seen that the particles from a chemical recovery facility (location $\mathrm{E}$ ) favors diameters less than 1 micron. This contrasts with the R\&D and fabrication facilities which, on the average, have a peak between 1 and 5 microns. "These measurements were taken before the exhaust filter banks, which consist of highefficiency particulate air (HEPA) filters. The work reported in Ref. 38 also included experimental measurement of the effectiveness of HEPA filtration systems in collecting these particles. Because HEPA's have a size dependent collection efficiency, they affect the size distribution of routine emissions. In major accidental releases, they would also affect size distributions in the same way, assuming that the integrity and efficiency of the filter banks is not impaired. These filters normally permit maximum penetration for particles size in the vicinity of 0.4 microns, which is in the range most effectively deposited in the pulmonary region. Substantially larger particles are deposited in the tracheobronchial region or the naso-pharyngeal passages, where they are available for transfer, via muco-cilial action, to the upper gastrointestinal tract, from which they are removed from the body with high efficiency. ${ }^{40}$ However, it is important to realize that regulations coverning release and maximum permissible concentrations for plutonium and uranium do not include any explicit size information. From a practical point of view, one does not expect very large particles (say 10 microns and up) to escape very easily from the site of production, nor in fact - to remain airborne for long if they do escape. This fact, together

* These results are consistent with measurements made by other workers, particularly at fuel fabrication facilities. Some of this work was recently reported in Ref. 39. 


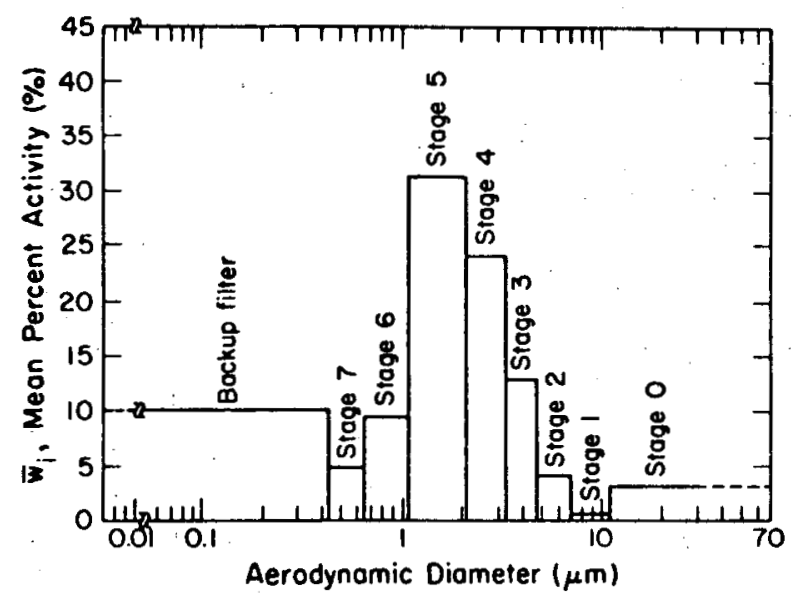

Location $A$, Research and Development,

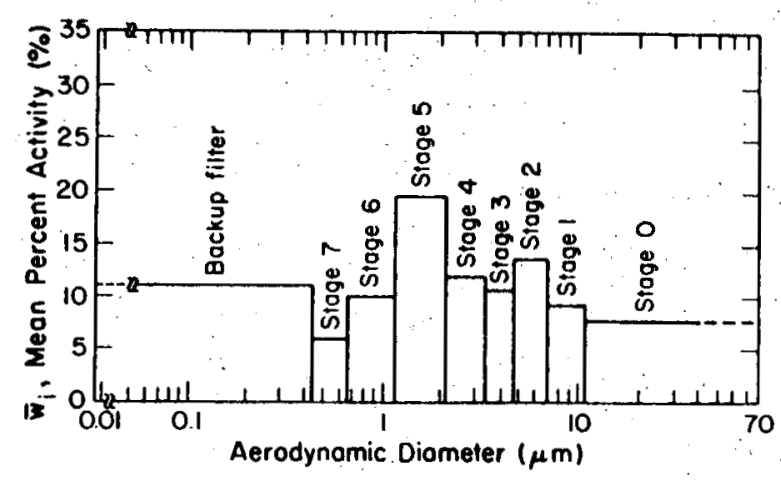

Location B, Research and Development, ${ }^{238} \mathrm{Pu}$

${ }^{238} \mathrm{Pu}$ and ${ }^{239} \mathrm{Pu}$

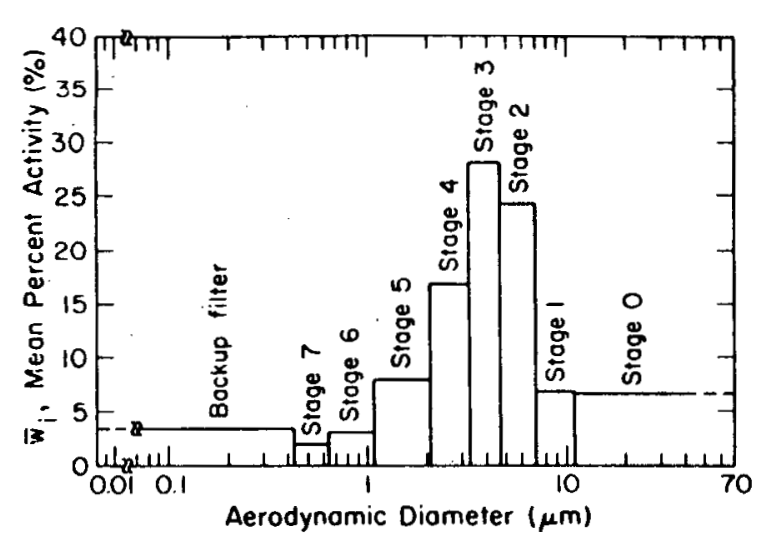

location C, fabrication, ${ }^{239} \mathrm{Pu}$

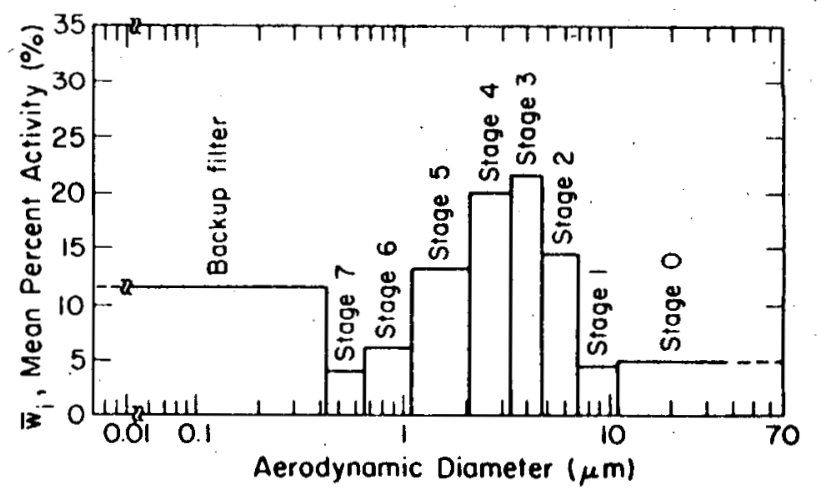

location 1), labrication, ${ }^{230} \mathrm{Pu}$

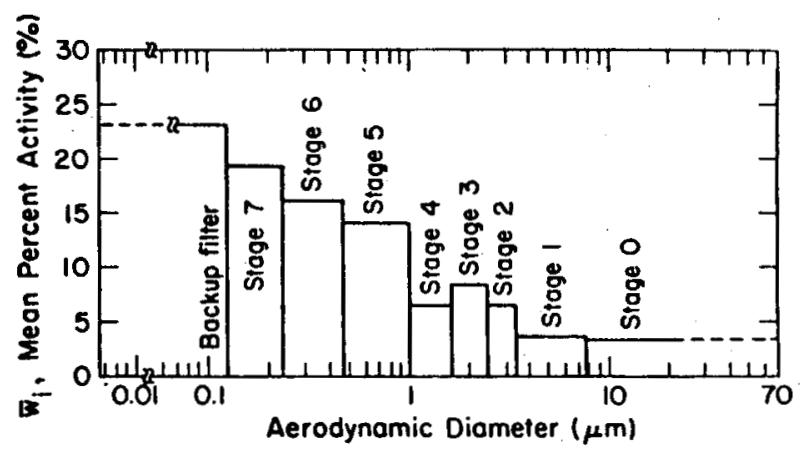

1.ocation It, Chemical Recovery, ${ }^{239}$ Pu

Fig. 3-12. Plutonium aerosol size characteristics as determined by radiometric analysis of all impactor samples taken at the specified location. The facility types and plutonium isotopes handled are indicated (From Ref. 38.) 
with the results cited above on size distribution of produced particulates and on HEPA filter efficiencies, make it reasonable to presume a substantial percentage of any release is in the biologically significant range.

In monitoring for releases, then, care must be taken that the collection and measurement technique is responsive to the biologically significant range, and preferably to the entire range. The size dependence of collection methods is discussed immediately below. Other parts of the monitoring procedure can depend on size, but how critically is difficult to determine. For example, the effectiveness of chemical leaching or dissolution of particulates in a bulk sample can depend on physical properties such as particulate size, how firing took place, etc. ${ }^{11}$ In alpha spectroscopy, energy loss in the particulates themselves can significantly affect energy resolution at the larger sizes. (Loss to a 1 micron thickness of plutonium or uranium dioxide is about $10 \mathrm{keV}$. At sizes much larger than this such losses become a significant contributor to the peak width in any high resolution system. It is of no concern in the typical system with a resolution of hundreds of $\mathrm{keV}$.)

For air monitoring, which is the most important aspect of monitoring releases from nuclear facilities, the basic element of most techniques is an air filter. These occur as two basic types: membrane filters with many sma11 holes through the membrane, so that the hole size determines the maximum size of particles passing through, and fiber filters formed of many fibers which collect particulates by either flow interception, inertial impaction, or thermal diffusion. One example of the latter is a HEPA filter, which is made of glass fibers. In an air cleaning system, such as those employing HEPA filters, the details of filtering efficiency are an important consideration, even though measured efficiencies are very high: greater than 99.9\%. These systems should clean the air as we11 as possible. However, when the filter is to be used to collect a sample for monitoring purposes, the difference between $99 \%$ and $100 \%$ efficiency is not significant. For the particle size range for which sampling filters are designed, they are satisfactorily efficient. As a result, the more difficult questions on air sampling have to do with the manner of collection of the air which is to be passed through the filter, and with subsequent treatment of the filter material.

In most monitoring situations, the intention is to determine the total anount of the substance of interest that is contained in the medium being monitored. In the case of air, plutonium and uranium are present in the form of particulates and, ordinarily, the sampler is intended to collect a percentage of these particulates that is independent of their size. For a stack monitor, this is typically 
accomplished by an "isokinetic" probe, one which draws off a sample air stream at the same velocity as the effluent air stream being sampled. The result is that no size selection occurs, at least in the range of interest. The material collected on the filter is then a measure of the total activity present, independent of the size distribution.

In other circumstance, it may be important to segregate particles by size. The most basic reason for such segregation would be that the size distribution is to be determined by the measurement. An alternative purpose of some importance is to discriminate against particles in some size range, either because they are of no significance, say biologically, or because they contain substantial background activities.

A variety of sizing techniques are available, but one that is most often used, because it can be implemented in a way that deposits different size fractions on convenient monitoring substrates, is the "cascade impactor". Each stage of this device makes use of the fact that, if an air stream is forced to change its direction, particles of sufficiently high momentum (which is proportional to mass for a given velocity) tend to escape from the stream. A sequence of such stages, with decreasing particle-diameter cut-offs, can bring about the type of scparation desired in monitoring intended to ascertain the particle size distribution. This is, for example, a technique that was used in the plutonium particulate measurements of references 38 and 39 .

The same principle may be employed simply to discriminate against an unimportant or irrelevant size range. For example, the particle sizes that are most apt to be deposited in the pulmonary region are those with diameters less than about 1 micron (for dense particles such as those consisting of plutonium or uranium dioxide). An impactor may be used to separate out the larger particles. Such a device has been used, for example to separate organic particulate matter in ambient air into respirable and non-respirable fractions. ${ }^{42}$ In this case, the device was a "virtual" impactor in which the incoming air stream is separated into two fractions, one of which contains, predominately, large particles, the other of which contains only smaller particles. The two streams may then be passed through air filters, as desired, to collect the particulates for monitoring purposes. This technique has not often been applied to monitoring for plutonium or uranium. In fact, its converse has been used in order to discriminate against the sma11 particulates which are likely to carry most of the background. One such device 43 uses a solid state detector as an impacting surface on which 
particles with diameter larger than about 1 micron are allowed to collect. In this manner, the device can discriminate against the roughly $99 \%$ of radon daughter activity that is associated with smaller particles. The detector surface would have to be cleaned regularly, just as the air filter in other systems has to be replaced on a routine basis. This same technique has been applied to more general radiation monitoring situations. In general, though, it is important to realize that it is often the smaller particles that are most biologically significant and that an impactor operated in this manner may therefore discriminate against the most important fraction unless care is taken that the size division is made at a very sma1l diameter.

Provided attention is given to this last consideration, an impactor could be generally useful in the typical monitoring situation. It is interesting to note that it can eliminate the need for an air filter, although it is still possible (and advisable in many situations) to collect the large size fraction on such a filter. The device would then be a virtual impactor in which one (or both) of the air streams is collected on a filter (or filters). This could greatly reduce the background due to radon daughter activity, and thus significantly improve the current generation of alpha monitors. However, in view of the observed size distributions of plutonium aerosols, for which significant percentages of the activity is contained in particles of submicron size, care would have to be taken that the cut-off was sufficiently small.

In one new development discussed above, the direct inlet mass spectrometer, the question of radon daughters is not important, but the separation of most of the plutonium or uranium activity into a relatively small stream of air would be very beneficial. In this device, only a small flow rate can be allowed into the ion source if that source is to operate properly. This decreases the rate at which air can be sampled inless the plutonium (uranium) in a large volume of air is somehow preconcentrated into a smaller volume which is then introduced into the ion source. A virtual impactor can perform exactly this function. Concentration factors of at least 10 are obtainable in a single stage, 42 so that a two stage device could concentrate the larger plutonium particulates into a volume fraction that is a factor of 100 less than the volume taken as a sample. Thus, for example, a 1 liter per second sampler would have its activity concentrated into a $10 \mathrm{~cm}^{3} / \mathrm{sec}$ air stream, a flow that may be acceptable to the spectrometer ion source. The crucial question is whether an acceptably low size cut-off is achievable in a virtual impactor. At 1 iter/second (about 2 cubic feet per minute 
or 2 CFM), a lower cut-off of about 0.2 microns is practical for cascade (nonvirtua1) impactors sampling plutonium. (See, for example, location E in Fig. 3-12, where the cut-off is $\approx 0.12$ microns at 2.75 CFM.) It would be interesting to know whether this is possible in a virtual impactor operating at atmospheric pressure. Building a virtual impactor with a submicron cut-off would probably require jets with such a sma11 diameter, and hence such a small flow rate, that a multiplejet design would be necessary for total sampling rates of 1 iter/second. If such a device could be designed for placement at the inlet of the mass spectrometer ion source, it could sample as great a volume of air as an ordinary "continuous alpha monitor", but it would respond to the collection of any particle immediately. The particularity of airborne activity would still be a major difficulty for this device, as for the usual sampler. For example, if the air contains $1 \mathrm{MPC}$ (general public) of plutonium 239 in the form of 1 micron diameter particles, there is, on the average, only one particle per 10 cubic meters or $10^{4}$ liters. Sampling at 1 iiter/sec would only collect 1 particle every. $10^{4}$ seconds, about 3 hours. The effective sensitivity would then be approximately 3 (general public) MPC-hours. Since basically any particulate will give a signal on the mass spectrograph, the sensitivity decreases in proportion to the volume of the particles or the cube of their diameter. Moreover, if expressed in 40 hour occupational MPC's, the sensitivity for 1 micron ${ }^{239}$ Pu particles is about $(1 / 40) \times 3 \mathrm{MPC}$-hour $\simeq 0.1 \mathrm{MPC}$-hour, much more sensitive than the typical claims, often optimistic, for commercial alpha air monitors. (Remember that this presumes an effective sampling rate of 1 liter/sec.) However, there is still no doubt that the discrete character of particulate plutonium constitutes a significant limit on the ultimate sensitivity of any air monitor. For a specified postulated particle size, this. limit becomes more stringent as the activity per unit mass increases. As a result, "reactor grade" plutonium, with a specific activity that exceeds that of ${ }^{239} \mathrm{Pu}$, would have fewer particles corresponding to any given activity concentration than would ${ }^{239} \mathrm{Pu}$. On the other hand, activity in which ${ }^{233} \mathrm{U}$ is a prime component would more easily be monitored via mass spectrographic methods than would plutonium.

Sampling considerations are also important for monitoring in media other than air. However, in such situations, it is not the physical technique that is critical, but rather the choice of sampling location and frequency - continuous samplers are not usually practical. Reference 4 includes a discussion of sampling techniques. In recent work ${ }^{44}$ an attempt is made, even in soil measurements, to use methods which are most sensitive to the biologically significant particle fraction.

Substantial work (see, for example, ref. 45) is being done on the biological and measurement aspects of plutonium and other types of particlate matter. 


\section{REFERENCES for Section 3}

1. T. H. Pigford and K. P. Ang. "The Plutonium Fuel Cycles"!, Health Physics 29, 451-468 (1975).

2. C. M. Lederer, J. M. Hollander, and I. Perlman, Table of Isotopes (6th ed), Wiley, N.Y., 1967.

3. Survey of Instrumentation for Environmental Monitoring, Lawrence Berkeley Laboratory Report LBL-1, Vo1. 3, Radiation, October 1973.

4. Radionuclides in the Environment: Measurement and Monitoring, IEEE Transactions on Nuclear Science, Vo1. NS-23, 1186-1223 (1976).

5. W. A. Phillips and C. L. Lindeken, "Plutonium Alpha Air Monitor Using a Solid State Detector," Health Physics 9, 299 (1963).

6. R. G. DelPizzo, J. B. Owen, and E. A. Putzier, "Rapid Estimation of Nnericium-241 Content in Plutonium," Health Physics 18, 725 (1970).

7. E. Tanaka, S. Iwadate, and H. Miwa, "A High-Sensitivity Continuous Plutonium Air Monitor," Health Physics 14, 473 (1968).

8. J. H. Spaa, "A Rapid Indicating Instrument for the Stepwise Measurement of Airdust Radioactivity," Health Physics $\underline{4}, 25$ (1960).

9. M. O. Rankin, "The Use of Coincidence Counting Techniques for Analyzing Low Level Plutonium Contamination on Filters," Nucl. Inst. and Methods 24, 221 (1963).

10. E.D. Gupton, "Alpha Air Monitor for Plutonium-239," Oak Ridge National Report ORNL-TM-2011, 1967.

11. J.W. Healy, "Problems of Plutonium Measurement in the Environment," IEEE Trans. Nuc1. Science NS-19 (1), 219 (1972).:

12. International Atomic Energy Agency, Rapid Methods for Measuring Radioactivity in the Environment, IAEA, Vienna (1971), comment p. 199 by C. W. Si11.

13. J.H. Harley, ed, HASL Procedures Manua1, USAEC Heal th and Safety Laboratory, Report HASL-300, 1972, updated to 1976.

14. C.W. Si11, K. W. Pupha1, F. D. Hindman, "Simultaneous Determination of AlphaEmitting Nuclides of Radium Through Californium in Soil", Analytical Chemistry 46, 1725 (1974).

15. N. A. Talvitie, "Radiochemical Determination of Plutonium in Environmental and Biologica1 Samples by Ion Exchange," Anal. Chem. 43, 1827 (1971).

16. T. Tomitani and E. Tanaka, "Large Area Proportional Counter for Assessment of Plutonium Lung Burden," Health Physics 18, 195 (1970).

17. L. B. Magnusson, "Intensities of X-rays and Gamma Rays in ${ }^{241}$ An A1pha Decay," Phys. Rev. 107, 161 (1957). 
18. L, A. Kroger and C. W. Reich, Nuclear Physics A259, 29 (1976).

19. H. L. Beck, J, E, Mclaugh1in, and K, M. Miller, "In Situ Determinations of Environmental Plutonium and Its Related Nuclides", IEEE Transactions on Nuclear Science, Vol. NS-23, 676 (1976).

20. J. F. Tinney, J. J. Koch, and C. T. Schmidt, "Plutonium Survey with an X-ray Sensitive Detector," Lawrence Livermore Laboratory Report UCRL-71362, 1969.

21. B. S. Burton and M. N. Anastasi, "Development and Construction of an Instrument to Detect Fissionable Materia1s," Air Force" Weapons Laboratory, Report AFWL-TR-71-145. Report available from Nuclear Chicago Corp., 9101 Research Blvd., Austin, TX 78766.

22. A. J. Ahlquist, C. J. Umbarger, and A. K. Stoker, "Recent Developments for Field Monitoring of Alpha Emitting Contaminants in the Environment", at the July 27, 1976 San Francisco Meeting of the Health Physics Society.

23. J. J. Koranda, P. L. Phelps, L. R. Anspaugh, and G. Holladay, "Sanpling and Analytical Systems for Measuring Environmental Radioactivity," p. 587 of Ref. 12.

24. J. A. Kirby, L. R. Anspaugh, P. L. Phelps, G. A. Armanțrout, and D. Sawyer, "A Detector System for In-Situ Spectrometric Analysis of ${ }^{241} \mathrm{Am}$ and Pu in Soil", IEEE Transactions on Nuclear Science, Vo1. NS-23, 683 (1976).

25. H. L. Beck, W. M. Lowder, and J. E. McLaugh1in, "In-Situ External Environmental Gamma-Ray Measurements Utilizing Ge(Li) and $\mathrm{NaI}(\mathrm{T} 1)$ Spectrometry and Pressurized Ionization Chambers" in IAEA "Rapid Methods for Measuring Radioactivity in the Environment", IAFA, Vienna, 1971.

26. See numerous references (pp. $154 \mathrm{ff}$ ) in IEEE Transactions on Nuclear Science, Vo1. NS-23, 1976.

27. W. H. Tyree and R. W. Bistline, "Plutonium-239 Detection in Vivo with a Geranium-Lithium Array," Rocky Flats Report RFP-1488, 1970.

28. R. W. Bistline and W. H. Tyree, "A Comparison of Low-Energy Photon Detection for Plutonium and Americium Wound Counting," Rocky F1ats Report RFP-1068, 1967.

29. W. H. Tyree, R. B. Falk, C. B. Wood, and R. W. Liskey," A High-Purity Germanium Detection System: for in vivo Measurement of Americium and Plutonium", Rocky Flats Report RFP-2525, in press.

30. E. I. Hamilton, "The Concentration of Uranium in Air From Contrasted Natural Environments," Health Physics 19, 511 (1970).

31. S. Amie1, "Analytical Applications of Delayed Neutron Emission in Fissionable Elements," Ana1. Chem. 34, 1683 (1962). 
32. F. S. Goulding and J. M. Jaklevic, "Photon-Excited Energy-Dispersive X-Ray Fluorescence Analysis for Trace Metals," Annual Reviews of Nuclcar Science 23, (1973).

33. R. D. Giaque, F. S. Goulding, J. M. Jaklevic, and R. H. Pehl, "Trace Element Determination with Semiconductor Detection X-Ray Spectrometers", Analytical Chemistry $\underline{45}, 671$ (1973).

34. Pacific Northwest Laboratory Annual Report for 1975 to the ERDA Division of Biomedical and Environmental Research, Report BNWL-2000, Part IV, p. 33, Feb. 1976.

35. C. M. Boyd and O. Menis, "Coulometric Determination of Uranium (IV) by Oxidation at Controlled Potentials," Ana1. Chem. 33, 1016 (1961).

36. W. D. Shults and P. F. Thomason, "Controlled-Potential Coulometric Determination of Copper and Uranium," Anal. Chem. 31, 492 (1959).

37. H. E. Zittel and L. B. Dunlap, "Polarographic Determination of Uranium (IV) in Sodium Tripolyphosphate Supporting Electrolyte," Ana1. Chem. 34, 1757 (1962).

38. J. C. Elder, M., Gonzales, and H. J. Ettinger, "Plutonium Aerosal Size Characteristics", Hea1th Physics 27, 45-53 (1974).

39. G. J. Newton, O. G. Raabe and S. V. Teague, "Plutonium Aerosols Inside a Safety Enclosure at a Mixed Oxide Reactor Fuel Fabrication Facility", Meeting of Health Physics Society, San Francisco, June 27, 1976.

40. Report to Committee II of the International Commission of Radiological Protection from the Task Group on Lung Dynamics, "Deposition and Retention Models for Internal Dosimetry of the Human Respiratory Tract," Health Physics 12, 17.3-207 (1965).

41. W. B. Seefeldt, W. J. Mechan, and M. J. Steindler, Charaćterization of Particulate Plutonium. Released in Fuel Cycle Operations, Argonne National Laboratory Report ANL-75-78, May 1976:

42. B. W. Loo, J. M. Jaklevic, and F. S. Goulding, "Dichotomous Virtual. Impactors for Large Scale Monitoring of Airborne Particulate Matter," Lawrence Berkeley Laboratory Report LBL-3854, May. 1975.

43. G. K. Rusch and W. P. McDowe11, "The 2PR-9 Airborne Plutonium Monitoring System", IEEE Transactions on Nuclear Science, Vol NS-23, 690 (1976).

44. C. J. Johnson, R. R. Tidba11, and R. C. Severson, "Plutonium Hazard in Respirable Dust on the Surface of Soil", Science 193, 488 (1976).

45. Inhalation Toxicology Research Institute Annual Report 1974-1975, Lovelace Foundation Report LF-52, December 1975. 
4. CURRENT PRACTICE: MONITORING INSTRUMENTATION, PROGRAMS, AND PROCEDURES

Instruments or techniques, and the manner in which they are used, are chosen in light of the objectives of a particular monitoring program. Possible program objectives were discussed in Section 2.4, and specific techniques in Section 3 . In this section, we will summarize the extent to which individual techniques have been applied and give examples of their implementation at both commercial and federal facilities. We will also discuss procedural and system aspects of implementation. Possibilities for improvement in current practice, including development of more effective techniques, are discussed in another section (1.2). 4.1 Techniques: Availability, Cost, and Extent of Application

For most of the techniques discussed in Section 3 , a working system may be assembled from commercially available components. In some case, pre-assembled systems (referred to below as "integral" units) may be purchased on either a routine or special order basis. In the discussion of individual systems below, the major components are specified and the approximate costs, within about a factor of 2, are given in 1976 dollars for systems purchased as components or, where available, as integral units; these costs are given to indicate the degree of financial commitment required. (The technical aspects of these systems, often illustrated by block diagrams, are discussed in Section 3. Photographs and references to many manufacturers are given in Ref. 1.) Final1y, for each technique, a comment is made on the importance of that technique as measured by the extent to which it is actually applied in monitoring of nuclear facilities.

The most basic categorization of instruments which may be used is source versus environmental monitoring, since both programatic objectives and the instruments themselves may often be classed in this way. As in Section 3, we begin with air monitoring, first discussing techniques which are primarily intended for source monitoring and then leading into techniques for environmental monitoring.

AIR MONITORING - based on filter collection and primarily for source monitoring

A common feature of most of these systems is the equipment for collection of air samples - a comment on this equipment is given under gross alpha monitoring.

Gross alpha counting -

equipment requirements -

collection: pump for air filter always required - commercially available (cost $\simeq \$ 300)$. Filter holder and sampling probe 
form depend widely on application - cost may easily vary from a small portion to a major part of the cost of the entire system, including detection (i.e., from $\$ 50$ to thousands of do11ars).

detection: scintillatór-photomultiplier or semiconductor detector, amplifier, counter - all components commercially available with a total cost $\simeq \$ 1000$.

integral units (with or without pump included) $\simeq \$ 1500$.

Alpha counting is the most basic form of air monitoring and has, usually, no provision for discrimination against background. It is often used in source monitoring (and in occupational monitoring), where a measure of the gross alpha activity may be useful if operational conditions preclude overwhelming radon daughter backgrounds. A wide range of components, at relatively low cost, is available for gross alpha monitoring. Moreover, the results are unlikely to be affected by changes in adjustment of voltages, amplification, etc. As a result, this type of detection system requires a minimum of human attention. Units can be assembled into a sma11 amount of space (1 cubic foot or 1ess). Alpha spectroscopy - semiconductor detector with direct counting from the

filter.

equipment requirements -

collection: same as for gross alpha

detection: semiconductor detector, amplifier, single channel analyzers, compensator (prescaler or the equivalent), counter - a11 components commercially available: with total cost $\simeq \$ 1500$.

integral units (with or without pump included) $\approx \$ 2000$.

Alpha spectroscopy is the most widely used method for source (and occupationa1) monitoring. With background subtraction techniques, it can discriminate substantially against interfering activities. The components are genera11y available, and little attention is required to adjust the single channel analyzers. Acquisition of the data requires no operator intervention from collection to readout. The units can be assembled into volumes of about 1 cubic foot. Lifetime analysis -

equipment requirements -

collection: same as for gross alpha detection: scintillator or semiconductor detector, amplifier, counter, time control, and readout - al1 components can be purchased 
commercially, total cost $\approx \$ 2000$.

integral units: not usually available.

Components of simple versions of this system are similar to those of a gross alpha system, and a sophisticated system may be similar to a spectroscopic system. However, the lifetime analysis itself, in which the activity fractions with differing half-lives must be separated by some data analysis technique, requires either sophisticated operator action or the equivalent of an automated computer analysis (as discussed in section 1). As a result, this technique is not often used and is not available in integral commercial units. Associated $\alpha-\beta$ measurements -

equipment requirements -

collection: same as for gross alpha

detection: two detectors, amplifiers, coincidence unit, compensator (prescaler), counter - $\simeq \$ 2000$.

integral units: not usually available.

The associated $\beta$ technique is not widely used. Performance is not superior to alpha spectroscopic systems, and the technique requires greater care in making the background subtraction because of a dual detector system. However, no data reduction is required; the result is simply read out.

Radiochemical analysis (air) -

equipment - similar to gross alpha or alpha spectroscopic techniques as above except that between the collection and detection the filter must be subjected to complex chemical, and sometimes electrochemica1, preparation. Requires a chemical laboratory, the cost of which depending on its sophistication - will equal, and perhaps exceed, the cost of collection and detection systems. Detection system cost will probably be $\simeq \$ 3000$, assembled from component umits. A11 analytical and detection equipment are commercially available.

Radiochemical analysis has personnel requirements an order of magnitude greater than the direct counting techniques described above, basically because the latter do not involve wet chemistry. Because of this personnel commitment, and indeed the time required to obtain a measurement from a single sample, radiochemical analysis is only performed routinely at large installations or at central laboratories which serve the smaller facilities. Radiochemical analysis followed by alpha spectroscopy has greater inherent sensitivity than the direct counting techniques; but, because of the one to three days required for analysis, it is 
most often used for long-term environmental sampling, or as a regular check on source monitoring by the direct counting methods.

High-volume environmental or source sampling -

equipment - since this usually involves radiochemical analysis preceded by a high-volume field or stack sampler, the equipment and cost are changed only by the smali incremental cost (which may add $20 \%$ to the full cost of the technique) of the sampler.

High-volume sampling is usually used in suspect environmental areas, perhaps in the immediate surroundings of a nuclear facility, or in special source or environmental testing.

General radiochemical analysis (followed by alpha spectroscopy) of soil water, and biological samples -

These techniques, as for radiochemical analysis (air) and high-volume air sampling, are used for sampling with high sensitivity, in this case principally of the general environment and areas surrounding facilities. Analysis is often performed at central laboratories. SURVEY TECHNIQUES -

FIDLER - integral units - principal availability on special order. Cost $\approx \$ 4000$.

Unit has been used primarily for survey of products of nuclear weapons programs, but has more general application.

Ge(Li) Survey Instruments - al1 components (detector, amplifiers, multichannel analyzer, data analysis) commercially available - total cost $\approx \$ 10,000$.

Must be assembled by user or contractor.

Not often used except at larger nuclear facilities, due to cost and personnel sophistication required:

phoswich - Detector and electronic components commercially available.

Cost $\simeq \$ 6000$.

Occupational personnel survey, not for release monitoring per se. INDUCED ACTIVITIES AND MASS SPECTROSCOPY -

Monitoring techniques based on any signature other than decay radiation requires more extensive or more sophisticated equipment than the methods described above. Since they are rarely used for routine monitoring of plutonium or uranium, we do not describe their cost and equipment requirements individually. The basic equipment for most of these techniques costs from $\$ 10,000$ to $\$ 30,000$ and - if a neutron generator must be included - these costs are usually considerably higher. As a result, most analysis of this kind would be on a service basis, performed at large laboratories. An obvious exception to this is direct inlet mass spectroscopy, 
which is specifically designed for on-line measurement and readout, of all these techniques, however, the direct inlet spectrometer is most clearly in the development stage.

\subsection{Release monitoring at commercial and federal nuclear facilities -}

We briefly give some examples of current uranium and plutonium monitoring practice within a number of nuclear facilities, at their exhaust stacks, and in their immediate surroundings. This survey is not comprehensive, but only. indicative of current practice. In many cases the monitoring method used is not specific to uranium and/or plutonium, but is applicable to other radionuclides; the method may in fact be designed specifically for other activities, which - in the particular facility - may be emitted in more significant amounts than uranium or plutonium.

The primary interest from the point of view of monitoring and control by public agencies, either at the state or federal leve1, is comercial nuclear power, as distinguished from research and/or weapons facilities. There is, however, some overlap between the two types of facility, since substantial portions of the nuclear fuel cycle are federally operated and, in certain categories, such as chemical recovery (reprocessing), no privately-held facilities are currently operating.

COMMERCIAL FACILITIES

Fabrication plants - typically employ alpha monitors, both for uranium and plutonium, for occupational and source monitoring, and send environmental samples, such as soil or vegetation or high-volume air samples, to independent laboratories for analysis.

Exxon Nuclear Fuel Fabrication Plants (Richland, Washington) uranium and mixed oxide facilities - have continuous alpha monitors and separate filter holders, both using alpha spectroscopic methods. Both types of systems are used both for in-facility monitoring and at the stack. ${ }^{2-3}$

Kerr-McGee (Cimarron facility) uranium facility - separate filter holders both in-facility and at the stack, with counting in an alpha spectroscopic unit at a central location. 4

mixed oxide facility - in addition to filter holders, has a continuous alpha monitor on the stack, using a (scintillator) alpha counting system. 4 
Reactors - ordinarily use gross alpha (as well as beta and gamma) monitoring on a continuous basis Alpha monitors are likely to be scintillation detectors. ${ }^{3}$ Primary effluents at such facilities are likely to be fission products rather than uranium or plutonium. Details of such systems can usua1ly be found in the Safety Analysis Report for the facility.

Reprocessing plants -

Allied-General Nuclear Services (Barnwe11, SC) - the Separations Facility will continuously monitor gross alpha (using a scintillator) and gross gamma. Other types of sample may be taken as needed for off-line analysis. 5,3

FEDERAL FACILITIES

Rocky Flats Plant (operated by Rockwe11 International) - this is a plutonium fabrication facility. Monitoring programs routinely use both continuous alpha monitors and separate filter holders; the latter filters are subsequently monitored for gross alpha (using a scintillator) and for specific nuclides by radiochemical analysis followed by alpha spectroscopy. ${ }^{6}$ An extensive environmental monitoring program is conducted at the facility.?

Los Alamos Scientific Laboratory (operated by Univ. of Calif.) -

DP - West - a piutonium research and development facility

Both in-facility and stack monitoring are performed with a system of separate filter holders, with off-line gross alpha counting, and with continuous air monitors, based on alpha spectroscopic techniques. (A new facility is being built that will use the same basic techniques.)

Idaho Fal1s Chemical Prócessing Plant (operated by Allied Chemical Corp.) For stack monitoring, uses scintillators, sensitive to gross alpha ; ${ }^{9}$ however, the primary activities emitted are fission products. Routine environmental monitoring for the entire Idaho Falls test facility is performed by the Health Services Laboratory. 10

Where no comment is made about a facility's environmental monitoring effort, no implication that such monitoring is omitted is intended. In fact, all of these facilities are required to perform and report on such monitoring, and these reports are often available in the open 1iterature. 10 
4.3 Procedural and system aspects of a monitoring program

The most basic components of a monitoring system are the fundamental collection and detection devices discussed in Section 3. However, an equally important aspect of monitoring is the manner in which the system handles and responds to data derived from these devices. A raw count rate is rarely significant in itself. The data must be processed in some way to compare it with some relevant standard, after which some response may be appropriate.

In any monitoring application, we can identify three levels at which there may be a need to process or handle data prior to use of derived information for subsequent action. The first level is at the detection device, where it is usually necessary to correct count rates for interfering backgrounds in order to derive a result for the activity of interest. This correction may involve only a simple electronic weighting and subtraction or it may involve a computer analysis of greater sophistication (such as '1ifetime analysis). The next level of sophistication in processing is to compare the result for the activity of interest with the relevant standards. In some applications, this comparison occurs directly at the instrument, which may even include visual or aural alarms. In other circumstances, such comparison may occur at some readout and control location, or even at some regulatory agency to which measured concentrations or emissions are reported. Whether comparison occurs at the instrument or 1ater, some provision must be made for a final level of processing, i.e., transfer of activity-level measurements to the readout-contro1 point just mentioned. This provision may take the form of a person or of a remote data handling system.

The distinction among levels of data handling just presented will vary depending on the particular monitoring technique employed and its intended app $1 \mathrm{i}$ cation. Moreover, utilization of the information, once received, will vary. It may be used for routine reporting or, if it indicates an abnormality, may elicit a response such as inspection or shutdown of the facility in question or even in more extreme cases - in warnings to populations which may be affected. These broader features of a monitoring program, and the related process of setting standards, are beyond the scope of this work. However, the data processing and handling sequence described above directly affects the effectiveness of monitoring and is discussed in Section 1.2, where possibilities for improvenent are considered,

A somewhat more subtle aspect of a monitoring system than the existence of the measurement instrumentation or of the data handling capability is the time response 
of the system and of the related administrative machinery. Emphasizing the former, a narrowly defined area that is well within the scope of this work, we have in a number of instances in Section 3 - noted the approximate length of time an instrument takes to detect some concentration of an isotope of interest. Decreasing this time in order to hasten response to any irregularity is a primary objective of instrumentation development, Such decrease in time corresponds to an increase in sensitivity and may be accomplished by introduction of new measurement techniques or by improvements in the present techniques, either at the detectors themselves or in the data processing, particularly in treating the data to remove background,

Improvement in sensitivity, in addition to decreasing the time taken to indicate emissions exceeding specified limits, results in the intrinsic ability to measure at lower levels. In many monitoring situations, the devices used give a reading of zero (within the measurement uncertainty) because of sensitivity limitations, even though the activities of interest are being emitted at some rate. Improving sensitivity lowers the concentration level at which the instrument gives a positive reading, and may even establish a nonzero baseline reading for normal operating conditions. This offers great advantages, since measurements can then give continuous information on emissions, rather than simply indicate emissions when the concentrations are above some lower limit.

One of the most striking characteristics of the operating monitoring systems listed in Section 4.2 is their range of sensitivity and sophistication: It is clear that although standards exist for radionuclide concentrations, little guidance exists for measurement capabilities. The range of instrumental sophistication exhibited by the various facilities results from some obvious factors. Some of these are the degree of concern over the plutonium or uranium being handled, the extent to which measurement of these materials interferes with measurement of other - sometime more significant - emissions, and the availability of fumds and personnel to implement and operate a monitoring system. These matters are a11 affected by improvements in the sensitivity, availability, and cost of instrumentation. The last matter, in particular, is an impetus to reduce the amount of personnel time required to operate a system. Finally, the general level of monitoring capabilities could be improved, not only by changes in the instrumentation itself, but by central availability of information on possible instrumentation and overall monitoring systems. 
REFERENCES for Section 4

1. Survey of Instrumentation for Environmental Monitoring, Lawrence Berkeley Laboratory Report LBL-1, Vol: 3: Radiation, Oct, 1973.

2. Information on montoring at these facilities is available in the license application and supporting materia1s, Docket \# $70 \div 1257$, Exxon Nuclear Fuel Fabrication Plants.

3. For generic information on these methods, see Section 3 of this report; for specific manufacturers; see Ref. 1.

4. Both of these facilities had discontinued operations by the beginning of 1976. See Ref. 3.

5. Final Safety Analysis Report for the Allied-General Nuclear Services Separations Facility.

6. K. J. Freiberg and C. G. Haynes, "Emission Monitoring Systems for Plutonium Facilities," Rocky F1ats Report RFP-2218.

7. See; for example, P. Krey et a1., "Plutonium and Americium Contamination in Rocky. Flats Soil, 1973", Health and Safety Laboratory Report HASL-304, March 1976.

8. W. J. Maraman, W. D. McNeese, and R. G. Stafford, "Confinement Facilities for Handling Plutonium," Health Physics 29, 469 (1975), and R. G. Stafford, private communication.

9. M. Arave, private communication.

10. See, for example, Environmental Monitoring at Major USERDA Contractor Sites," USERDA Report ERDA-54 (1974), August, 1975. 


\section{GLOSSARY}

(Although many of these terms have wider applicability than the present context, they are defined here in terms appropriate to this report.)

activity - see radioactivity; may be used loosely in referring to particular radioactive nuclide (s).

alpha emitter - a radioactive nuclide which decays by emission of an alpha particle.

alpha particle - the type of particle emitted from nuclei which is actually identical to the nucleus of a helium 4 atom; it consists of two neutrons and two protons.'

ambient level - the actual concentration at some particular time of a substance of interest in a specified medium.

background level - the ambient level in the absence of introduction of some change. One example is the average human radiation exposure (in, say, rem per year) in the absence of any increase caused by nuclear power. This exposure serves as "background" to the exposure after the introduction of nuclear power. As another example, an instrumental background is the portion of a measurement result that is not due to the radionuclide(s) of interest. This background may arise either from other radionuclides or from instrumental noise.

baseline - refers to a level with which various results will be compared; in monitoring for radionuclides, the baseline level for a given nuclide is often the normal concentration in which that nuclide would be found a monitoring program could then look for changes in the concentration.

beta particle - an electron or positron (a positively-charged electron) emitted from a nucleus.

body burden - the amount of radioactivity, usually of a specific radionuclide contained within a. human's body.

collection - the process of assembling a sample:

concentration or level - the amount of radioactivity per unit mass or volume.

counting statistics - refers to the statistical significance implied directly by the number of counts accumulated in a measurement; as an example, in a measurement where typically $\mathrm{N}$ events are observed and the individual events occur on some random basis (such as does the decay of any specified radioactive nucleus), the intrinsic uncertainty in that number of counts is the square root of $\mathrm{N}$. (That is, even if the same sample is being measured under the same conditions, the result is expected to vary from $\mathrm{N}$ by approximately

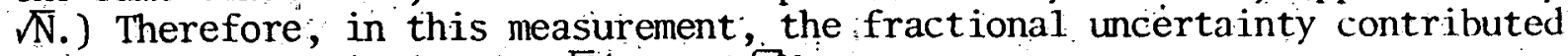
by counting statistics is $\sqrt{\mathrm{N}} / \mathrm{N}$ or $1 / \sqrt{\mathrm{N}}$.

Curie - an amount of radioactivity that yields $3.7 \times 10^{10}$ disintegrations per second daughter - a nuclide produced as a result of decay of a radioactive nuclide (the "parent"); may refer to any of the subsequent generations from a specified parent. 
dose - see exposure

electrodeposition - process whereby a current is passed through an ion-bearing solution and material of interest is deposited from the solution onto one of the electrodes.

electron volt (eV) - the amount of energy gained by a particle wi th one electronic charge when it is accelerated through an electrical potential of one volt; $1 \mathrm{eV}=1.6 \times 10^{-19}$ joules. $1 \mathrm{keV}=.1000 \mathrm{eV} ; 1 \mathrm{MeV}=1000 \mathrm{keV}=10^{6} \mathrm{eV}$.

excitation - the process of changing the state of a system from a low energy state (usually the "ground" or lowest energy state) to a higher energy state; "atomic" excitation consists of an alteration of the electronic structure, and nuclear excitation implies alteration of the internal structure of the nucleus.

exposure or absorbed dose - the amount of radiation received by an organism or material, usually given in terms of a unit. based on energy deposited per unit mass (see rad, rem).

fast neutrons - neutrons having enèrgy much greater than thermal energy. In the context of reactors, fast neutrons are those having energy comparable to their energy on emerging from fission events (i.e., on the average $2 \mathrm{MeV}$.)

fertile nuclei - nuclei which can be converted to fissile nuclei by absorption of neutrons, followed by radioactive decay; examples are $238 \mathrm{U}$ and $232 \mathrm{Th}$.

fissile nuclei - nuclei which can be induced to fission by slow or "thermal" neutron; examplès are $235 \mathrm{U}$ and $239 \mathrm{Pu}$.

fissionable nuclei - nuclei which can be induced to fission by neutrons of some (not necessarily thermal) energy; includes both fissile and fertile nuclei.

fission products - the major fragments resulting from fission; they are actually medium-weight nuclei such as ${ }^{137} \mathrm{Cs}$ and $90 \mathrm{Sr}$.

fuel cycle - the various operations (or facilities) for production, reprocessing, and disposal of fuel.

fuel element or assembly - the basic fuel module for insertion into a reactor. For most commercial reactors, an assembly consists of a bundle of long fuel rods, each of which is filled with a stack of cylindrical uranium dioxide pellets.

gamna radiation - electromagnetic radiation emitted from a nucleus.

half-1ife - the time during which half of the nuclei in a sample of a particular nuclide will decay spontaneously. 
ion - a basic atomic or molecular unit with a net non-zero charge, so that it is not electrically neutral; consists of an atom or combination of atoms with an excess or deficit of electrons, relative to the electrically neutral object.

ionization - the process of creating pairs of oppositely charged ions: often refers specifically to removal of electrons from an atom or molecule.

ion pair - two oppositely charged ions, usually formed from the same neutra] atom, molecule, or other medium (such as a semiconductor materia1); may refer to an ion and an electron or to a particle and a hole (as in a , semiconductor), in addition to two actual ions.

isotope - one of the (several) specific types of a given element. The element is specified by proton number; a particular isotope of that element is then specified by the mass (or neutron) number.

microprocessor - a device, based on one or a few large-scale integrated-circuit semiconductor çhips, which performs al1 the logical and control functions of a computer's central processing unit, including the basic arithmetic and word-handling functions.

mixed oxide - refers to nuclear fuel consisting of a mixture of uranium and plutonium dioxides.

noise, instrumental - spurious signals produced as a result of random, or at least uncontrolled, fluctuations in the instrument itself; such "noise" may constitute a background to the signals of interest.

nuclide - a particular nuclear type, specified by proton number and neutron number (the sum of which is the mass number, A).

particulate - referring to particle.

rad - the unit of exposure or absorbed dose, given as energy absorbed per mass of living tissue; ' $1 \mathrm{rad}=100 \mathrm{ergs} / \mathrm{gram}$ ( or $0.01 \mathrm{joules} / \mathrm{kg})$.

radiation - a generic term for the various emissions resulting from nuclear, atomic, or molecular processes; in the context of this report, it refers principally to the alpha, beta, gamma; and $x$-radiation emitted from atoms and nuclei.

radioactivity - the capability of certain nuclides to decay ("disintegrate") spontaneously to other nuclides, giving up energy and some specific type(s) of radiation in the process; the disintegration rate of a radioactive sample is measured in Curies.

range - the "average" distance (often defined via some precise arithmetic algorithm) traveled through some specified material by radiation of a specific type and energy. The range may be given by a linear distance (for example, in centimeters) or by a mass thickness (in grams $/ \mathrm{cm}^{2}$, for example). 
readout - the process of exhibiting the result of a measurement; this may involve printing the result, displaying it on an electronic module (such as a digital display or meter), plotting it on graph paper, etc.

release - in the context of this report, any entry of radionuclides from a fuel cycle facility into the general environment; applies to both routine and accidental emissions.

rem - an exposure unit which, for radiation of a specified type and energy, is proportional to a rad, but which takes into account the relative biological effectiveness of the radiation; the dose equivalent (in rems) $=$ dose (in rad) $x$ quality factor, which factor equals 1 for $x$-radiation and electrons, but which is larger for heavier particles.

resolution - the quantified ability of an instrument to distinguish differences in the parameter measured; energy resolution may, for example, be given in $\mathrm{keV}$ and refer to the instrumentally contributed energy width of a peak resulting from a radioactive source emitting radiation of a well-defined energy; time resolution, measured, for example, in nanoseconds, gives the intrinsic instrumentally contributed time width in a time (as opposed to energy) spectrum.

sampling - refers either to the process of taking an individual sample for measurement or to a systematic program of taking such samples to attain some monitoring objective. A sample itself may be collected on a filter, as is often the case in air sampling, or may be taken directly from the medium to be sampled, as in a solid or water sample.

solubility - the ease with which a material may be brought into solution.

specific activity - amount of radioactivity per unit mass for a particular nuclide or sample. Measured in Curies per gram or similar units.

spectroscopy - measurement of the specific value of some parameter (usually the energy, but may also refer to other parameters, such as time). The result may be given as a "spectrum".

spectrum - a measurement readout in which the parameter measured is displayed in effectively continuous fashion. For example, if an energy range is divided into small intervals, the "spectrum" resulting from some measurement would specify the number of events (such as measured nuclear particles) in each energy interval. Analogously, a time or frequency spectrum may result from other types of measurement.

tailings - the remant from any material processing, especially one in which a substance of particular interest is extracted from a larger amount of material, thereby leaving a less valuable remant behind. In the nuclear fuel. cycle, the term is applied to the ore tailings pile at a uranium mill and to the $235 \mathrm{U}$-depleted uranium stream at an enrichment plant.

thermal reactors - reactors which take advantage of increased fission cross sections : at low neutron energies by rapidly moderating neutrons to low energy through collisions with low-atomic-weight materials. 
thermal or slow neutrons - neutrons which have reached thermal' equilibrium with their surroundings, so that they are at the same temperature and have the same average energy as the molecules of the material containing the neutrons. This thermal energy is, for typical reactor temperatures, approximately $10^{-8}$ times the average energy of neutrons as they are released from fission.

thickness, mass - the thickness of material given in terms of mass per unit area (e.g., grams $\left./ \mathrm{cm}^{2}\right)$. For material of density (mass per umit volume), the mass thickness $\underline{\mu}$ is related to the linear thickness $t$ by the relation, $\mu=\rho t$.

thoron - the historical name for ${ }^{222} \mathrm{Ra}$.

uptake - the process whereby a living organism ingests or absorbs a radioactive. substance.

$x$-radiation - electromagnetic radiation emitted in the course of a change in the electronic (as opposed to nuclear) structure of an atom; often follows a change in the nuclear structure. 
Appendix A.' - A Selection of Standard and Guides -

As discussed in section 2,3, standards of several types are applicable to radiation and radionuclides in general and to uranium and plutonium in particular. Direct responsibility for licensing of nuclear facilities lies with the Nuclear Regulatory Commission (NRC), which functions in conformity with title 10 of the Code of Federal Regulations (10CFR). In 1icensing proceedings, the NRC enforces an "as low as is reasonably achievable" limit on individual facilities. 'The NRC: publishes a series of Regulatory Guides to give guidance to applicants for licenses on how the requirements of 10 CFR may be satisfied.

In addition to the general "as low as is reasonably achievable" standard, there are several more specific standards and guides promulgated by several committees and agencies:

Table A-1 selected from NCRP Report \#39, gives genera11y applicable exposure limits, consistent with those recommended by the ICRP (see Section 2.3).

Table A-1. Dose-1imiting recommendations

Maximum Permissible Dose Equivalent for Occupational Exposure

Combined whole body occupational exposure

Prospective annual limit -

Retrospective annual limit -

Long term accumulation to age $N$ years

Skin

Hands

Forearms

Other organs, tissues and organ systems

Fertile women (with respect to fetus)

Dose Limits for the Public, or Occasionally Exposed Individuals

Individual or Occasional -

Students

5 rems in any one year

10-15 rems in any one year

$(\mathrm{N}-18) \times 5$ rems

15 rems in any one year

75 rems in any one year (25/qtr)

30 rems in any one year (10/qtr)

15 rems in any one year. (5/qtr)

0.5 rem in gestation period

\section{5 rem in any one year}

0.1 rem in any one year

Population Dose Limits; averaged over a suitable sample of the general public Genetic

Somatic

Emergency Dose Limits-Life Saving Individual (older than 45 years if possible)

Hands and Forearms

Emergeńcy Dose Limits-Less Urgent Individua1

Hands and Forearms

0.17 rem average per year

0.17 rem average per year

100 rems

200 rems, additional (300 rems, tota1)

25 rems

100 rems, total 
An addition to $40 \mathrm{CFR}$ has been proposed by the Environmental Protection Agency and is presently under consideration: This addition would impose limitations on dose equivalent from facilities for the uranium fuel cycle and on emissions of specific radionuclides, such as. the transuranics (including plutonium-239). The proposed Part 190 is reproduced here as published in the Federal Register (p.23424 ff, May 29, 1975). (e) "Radiatlon" means any or all of the following: alpha, beta, grmma, or $x$ rays; neutions; and high-energy clectrons, prolons, or other atomic particles; but not sound or radio waves, nor visible, infrared, or viltraviolet llght.

(f) "Radionctive material" means ang material which enits radiation.

(f) "Uranium ore" is any ore which contains one-twenticth of one percent (0.05 percent) or more of urantum by weight.

(h) "Curie" (Ci) means that quantlly of radioactive material producing 37 billion nuclear transformations jer second. (One millicurie $(\mathrm{mCl})=0.001 \mathrm{Ci}$ )

(i) "Dose equivalent" means the yroduct of absorbed dose nind upproprinte ractors to account for difrerences in biological effectiveness due to the quality of radiation and ils spatial distribution in the body. The unit of dose equivalent is the "lem." (One millirem (mrem) $=0.001 \mathrm{rem}$.

(j) "Organ" means any humall orkan exclusive of the dermis, the epidernis, or the comea.

(k) "Gigawatt-year" refers to the quantity of electrical cnergy procluced at the busbar of $n$ generating shation. $A$ gigawatt is equal to one billion watts. A gigawatt-ycar is equivalent to the amount of enerky output represented by an average electric; power level of one gigawatt sustained for one year.

(1) "Menber of the public" means any Individual that can recelve a radiation dose in the gencral chviromment, whether he may or may not nlso be exposed to radiation in nu occupution associated with a nuclear fuel cycle. However, an individual is nol considered a member of the public during any period in which he is engaged in carrying out any operation which is part of a nuclear fuel cycle.

(m) "Regulatory Hrency" means the government agency resuonsible for issuing regulations rovernlng the use of sources of radintion or ladlonetive materials or emsssions therefrom and carrying out inspection and enforecment activities to assure compliance with such regulations.

Subpart B-Environmental Standards for the Uranium Fuel Cycle

$\$ 190.10$ Sinudarits for mormial mpera. iimu.

(a) The annual dose equivalent shall not excecd 25 millirems to the whole body. 75 milltrems to the thyrold, and 25 millirems to any other orran of any menber of the jublic as the result of exposure to plamiced dilschnrges of rudioactive materials, radom and its daughters excepted, to the general cuviroment from uraniun fuel cycle ojoritions and racliation from these orerations.

(b) The total quantity of racionctive naterials entering the general environment froin the entire urantum fucl cycle, per gigawatt-ycar of electrical energy produced by the fuel cycle, shall contaln less than 50,000 curles of krypton-85, 5 
millicuries of lodine-129, and 0.5 millicuries combined of plutallum-239 and other alpha-emitting trausuranlc radionuclides with half-lifes greater than one year.

\$ 190.11 Variance for unusual operalions.

The standards specified in $\$ 190.10$ may be exceeded if: (a) The refulatory agency has granted a variance based uyon its determination that a temporary and unusual operiting condition exists and continued operation is necessary to protect the overall societal interest with respect to the orderly delivery of electrical power, and

(b) Information delineating the nature and basis of the variance is made a matter of public record.
$\$ 190.12$ limotive date.

- (a) The standards in this Subpart, excepting those for krypton-85 and iodine129 , shall be effective 24 months from the promulgation date of this rule.

(b) The standards for krypton-85 and iodine-129 shall be effective January 1 , 1983.

[FR Doc.75-14017 Filed 5-28-75;8:45 am]

Finally, in Table A-2, we give an example of specified maximum permissible concentrations (MPC) for individual radionuclides. The table is taken from the National Bureau of Standards NBS Handbook \#69. (Even more specific 1imits, i.e., on each of isotope of these elements, are given in $10 \mathrm{CFR}$ Part 20. The NBS table further indicates how specified MPC's are associated with doses to individual organs.) 
Table A-2. Excerpted from National Bureau of Standards NBS Handbook \#69

Maximum permissible body burdens and maximum permissible concentrations of radionuclides in air and in waler for occupational exposure

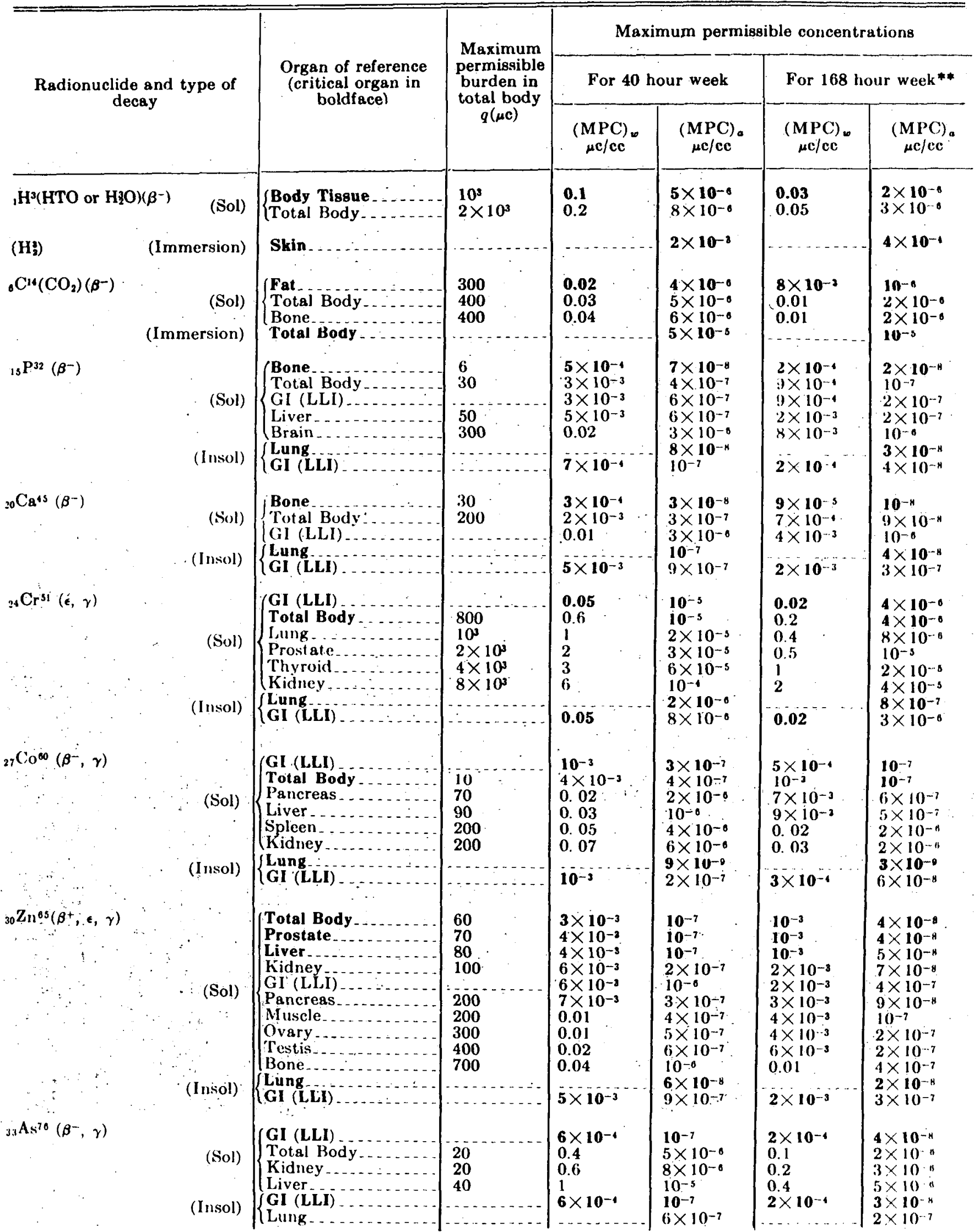

"The abbreviations GI, S, SI, ULI, and LLI refer to gastrointestinal truct, stomach, small intestines, upper largo Intestine, und lowor larko intestlü, respectively.

-It will be noted that the M PC values for the 168-hour week are not always precisely the sume multiples of the M PC for tho 40 -hour weok. Firt of thls is

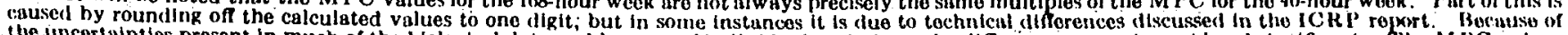

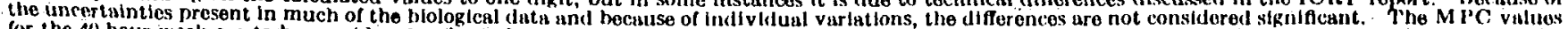

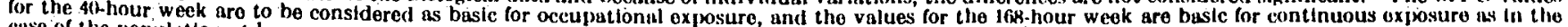


Table A-2. (Continued) Excerpted from Nationa1 Bureau of Standards NBS Handbook \#69

Marimum permissible body burdens and maximum permissible concentrations of radionuclides in air and in water for occubational exposure-Continued

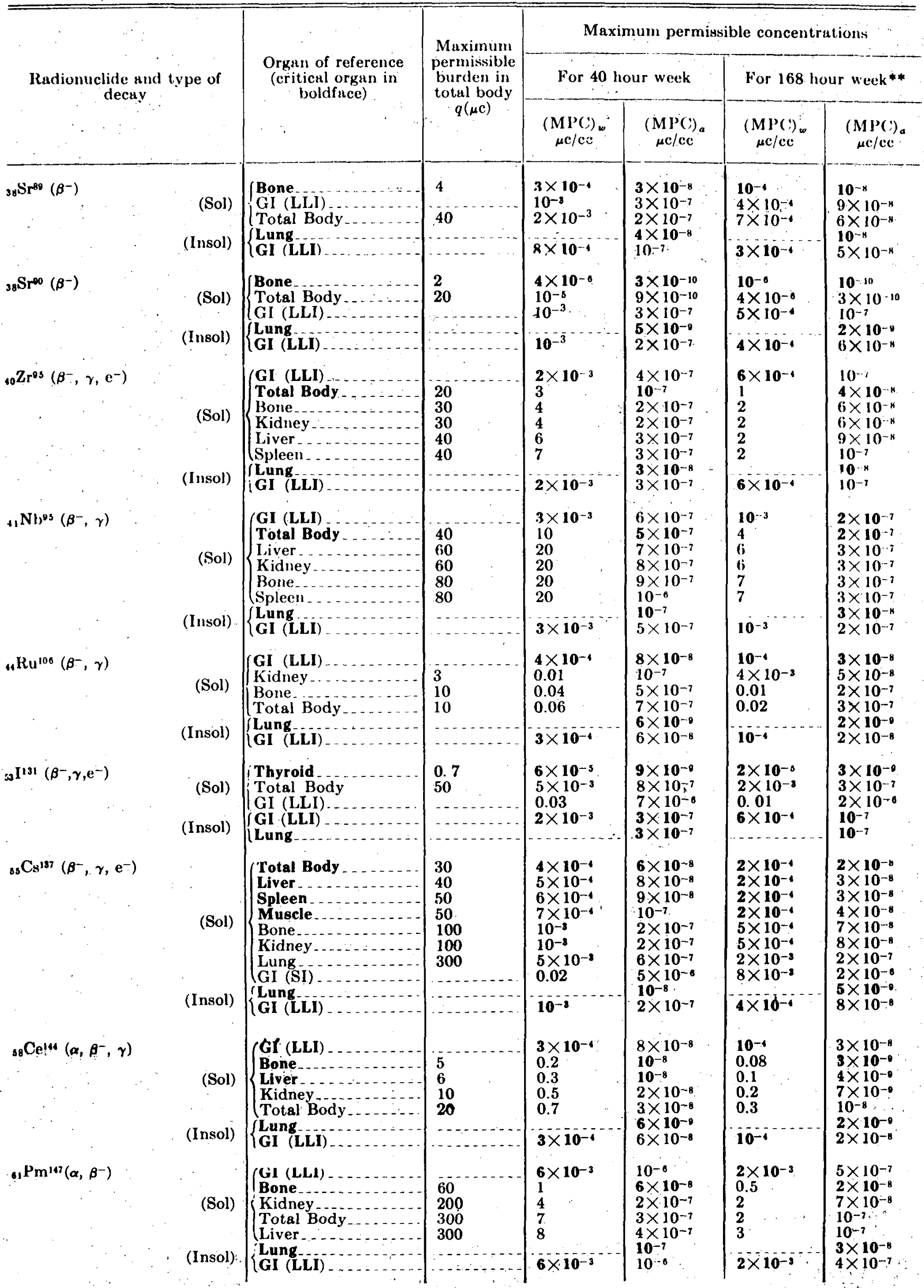


Table A-2. (Continued) Excerpted from National Bureau of Standards NBS Handbook \#69

Maximum permissible body burdens and maximum permissible concentrations for radionuclides in air and in water for occupational exposure-Contimmed

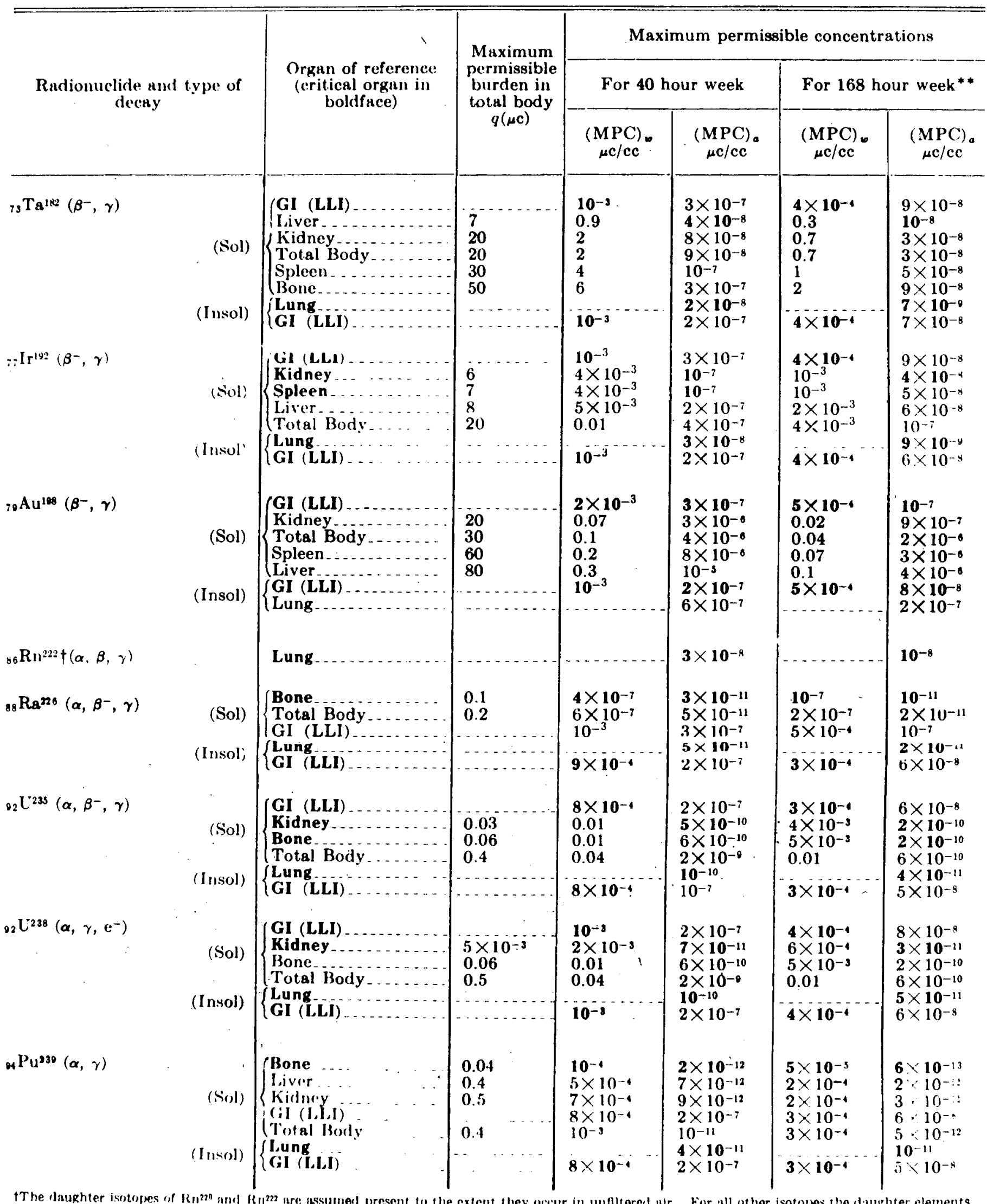

